\title{
The superspin approach to a disordered quantum wire in the chiral-unitary symmetry class with an arbitrary number of channels
}

\author{
Andreas P. Schnyder ${ }^{\mathrm{a}}$, Christopher Mudry ${ }^{\mathrm{b}}$, Ilya A. Gruzberg ${ }^{\mathrm{c}}$ \\ ${ }^{a}$ Kavli Institute for Theoretical Physics, University of California, Santa Barbara, California 93106, USA \\ ${ }^{\mathrm{b}}$ Condensed Matter Theory Group, Paul Scherrer Institute, CH-5232 Villigen PSI, Switzerland \\ ${ }^{\mathrm{c}}$ The James Franck Institute, The University of Chicago, 5640 S. Ellis Avenue, Chicago, Illinois 60637, USA
}

\begin{abstract}
We use a superspin Hamiltonian defined on an infinite-dimensional Fock space with positive definite scalar product to study localization and delocalization of noninteracting spinless quasiparticles in quasione-dimensional quantum wires perturbed by weak quenched disorder. Past works using this approach have considered a single chain. Here, we extend the formalism to treat a quasi-one-dimensional system: a quantum wire with an arbitrary number of channels coupled by random hopping amplitudes. The computations are carried out explicitly for the case of a chiral quasi-one-dimensional wire with broken time-reversal symmetry (chiral-unitary symmetry class). By treating the space direction along the chains as imaginary time, the effects of the disorder are encoded in the time evolution induced by a single site superspin (nonHermitian) Hamiltonian. We obtain the density of states near the band center of an infinitely long quantum wire. Our results agree with those based on the Dorokhov-Mello-Pereyra-Kumar equation for the chiralunitary symmetry class.
\end{abstract}

Key words: Disordered systems, Localization, Supersymmetry, Path integral, Mesoscopics PACS: 03.65.Pm, 71.23.An, 72.15.Rn, 71.23.-k, 11.30.Rd

\section{Introduction}

It has been 50 years since Anderson wrote his seminal paper that came to define the problem of Anderson localization, a tight-binding model (in three-dimensional space) for noninteracting quasiparticles subject to a static disorder (random uncorrelated on-site energies) [1]. The insight of Anderson was to realize that disorder, however weak, can have dramatic consequences on the nature of the eigenfunctions. In three dimensions, disorder turns extended states of the clean system into exponentially localized states when the characteristic kinetic energy of the extended 
states, measured with respect to edge of the band, is of the same order or smaller than the characteristic energy of the disorder.

For fermionic quasiparticles, this ratio can be tuned by changing the number of occupied single-particle states, i.e., by changing the chemical potential. The value of this ratio at which single-particle eigenstates are neither exponentially localized nor extended, if it exists, is called a mobility edge. The mobility edge realizes a quantum critical point, i.e., there exists a diverging length scale as it is approached [2, 3 , 田F]. On the metallic side of this continuous phase transition, disorder can be treated perturbatively thereby defining the diffusive regime. On the insulating side of this continuous transition, it is the kinetic energy that can be treated perturbatively [1.6]. A solid analytical grasp of the quantum criticality at a mobility edge remains an outstanding problem in Anderson localization.

The existence and characterization of a quantum critical point at a mobility edge for fermionic single-particle states was argued on the basis of a scaling hypothesis combined with perturbative calculations (see Refs. [2],5] for reviews). The tool of choice to implement the scaling hypothesis in a system of infinite size has been to encode the effects of weak disorder on the fermionic quasiparticles into an effective interacting field theory called a nonlinear-sigma model (NLSM). The space spanned by the fields of the NLSM, the target manifold, is determined by the intrinsic symmetries obeyed by the random microscopic Hamiltonian [7, 8, 9, 10, 11, 12]. These intrinsic symmetries are the presence or absence of the symmetry under time-reversal, spin-rotation, or charge-conjugation, respectively [12]. By demanding that the time-evolution generated by the random microscopic Hamiltonian is unitary and that it preserves the fermionic anticommutation relations, the target manifold of the corresponding NLSM belongs to one of the 10 classical symmetric spaces [13 in either their compact, noncompact, or supersymmetric incarnations [9]. The notion of 10 symmetry classes has thus emerged as a corollary to the scaling hypothesis, here implemented by 10 families of NLSMs. The local and global properties of a target manifold fixes the number of independent coupling constants that enters in a NLSM. From this point of view, a (nonexhaustive) classification of the mobility edges for fermionic quasiparticles in one, two, and three dimensions [14] into universality classes can be deduced from the curvature and the homotopy group of the 10 classical symmetric spaces 15, 16, 17, 18, 19, 20, 21, 22, 23, 24, 25, 26, 27, 28, 29, 30, 31, 32, 33, 3, 35, 36, 37, This construction of a NLSM starting from a random microscopic Hamiltonian relies on the existence of a diffusive regime [21]. However, mobility edges in $d=1,2,3$ dimensions are usually far away from the diffusive regime. The predictive power of NLSM beyond the existence of mobility edges is therefore severely limited by ones ability to study NLSM at their strong coupling fixed points.

The validity of the scaling hypothesis has been tested with the help of nonperturbative numerical calculations on random tight-binding or network models. The hypothesis of one-parameter scaling in quantum wires as well as the existence of mobility edges in $d=1,2,3$ dimensions for different intrinsic symmetries of the microscopic models is consistent with diverse numerical calculations in systems of finite-sizes (see Refs. [3.4.,5] for reviews). In this context, random network models play an important role [38, 39, 40, 41, $42,43,44,45,46,47,48,49,50]$. Consider for example the plateau transition in the integer quantum Hall effect (IQHE) (for a review see Ref. [51]). Chalker and Coddington (CC) have proposed a random network model to study the effects of disorder on the highly degenerate single-particle states in the first Landau level of a twodimensional electronic gas in a strong magnetic field when the cyclotron length is much smaller than the characteristic length over which the random potential varies significantly [38]. The CC network model simplifies greatly the study of the plateau transition in the first Landau level. For example, the position of the mobility edge that separates two integer Hall insulating states in the 
CC network model follows from a duality argument. Properties of the mobility edge in the IQHE can thus be simulated numerically for large system sizes and large statistical ensembles for the disorder with the help of the CC network model. In addition, the CC network model has been used as a regularization of the corresponding NLSM. Namely, the CC model has been mapped to a certain one-dimensional antiferromagnetic (super)spin chain, with the space of states being the tensor product of alternating highest and lowest weight infinite-dimensional irreducible representations (irreps) of a Lie (super)algebra, also loosely called "(super)spins", and the Hamiltonian (an infinitesimal version of the transfer matrix of the CC model) built out of generators of the same algebra. The (super)symmetry of the spin chain was the same as that of the target space of the NLSM [52,53, 54,55,56,57,58]. This was an important conceptual step in the sense that it provided the hope that the flow to strong coupling of the corresponding NLSM could be captured at the level of the (super)spin chain in a way similar as the flow from the $O(3)$-NLSM to the $S U(2)_{1}$ Wess-Zumino-Witten (WZW) conformal field theory is captured by the Bethe Ansatz solution to the quantum spin-1/2 Heisenberg chain [59]. Although, this hope proved ephemeral in the context of the plateau transitions (for a review see Ref. [57]), it lead to remarkable exact results when the same approach was used in the case of the two-dimensional Bogoliubov-de-Gennes (BdG) symmetry class with spin-rotation invariance but with broken time-reversal symmetry, and the corresponding network model [47,60,61, 62, 63, 64,

Unfortunately, this success story is the exception rather than the rule at the present. For example, consider the cases of spinless fermionic tight-binding models with either random real-valued or random complex-valued hopping amplitudes, whereby the hopping amplitude is restricted to connecting pair of sites with each site belonging to a different sublattice of a two-dimensional bipartite lattice. Such tight-binding Hamiltonians have the property that their spectra of energy eigenvalues are symmetric under charge conjugation, i.e., they are left invariant under sign reversal whatever the realization of the random hopping amplitudes. This is most easily seen by changing the sign of the single-particle eigenfunction for all sites belonging to one sublattice. As this property is also shared by the Dirac Hamiltonian in quantum chromodynamics for a given configurations of the gluonic gauge fields, it is called a chiral symmetry (chiral-orthogonal symmetry when the Hamiltonian is real-valued and chiral-unitary symmetry otherwise) [8]. In one and two dimensions, the band center in the chiral-unitary or chiral-orthogonal symmetry classes realizes a mobility edge separating two insulating phases very much in the same way as is the case with the plateau transitions in the IQHE.

The study of localization in the chiral-unitary symmetry class in one-dimensions was pioneered by Dyson [65]. Dyson found a diverging density of states on approaching the band center for a random microscopic Hamiltonian describing a single-particle hopping with a random amplitude between the nearest-neighbor sites of an infinitely long open chain. The band center realizes a quantum critical point that separates two insulating phases. This quantum critical point has been investigated using various methods such as a mapping onto a random $X Y$ model [66], solving a recurrence relation [67,68], solving a stationary Fokker-Planck equation [69], the Berezinskii diagram technique [0,71], solving a one-dimensional diffusion process with reflecting and absorbing boundary conditions at the left and right ends [72], respectively, solving the evolution equation of a transfer matrix [73, a mapping onto Witten's supersymmetric quantum mechanics [74,75], a mapping to Liouville quantum mechanics [76], and, at last, a mapping onto a spin-Hubbard-like model [77, 78, 79$]$. (We note here that in this case the spin-like model involves two sites with a highest and lowest weight representations of a Lie superalgebra paired in a supersymmetric fashion. In this setting one has to solve the problem of the two couples superspins, analogous to the "spin addition" in quantum mechanics, albeit more com- 
plicated due to the infinite dimensionality of the superspins.) At the band center, the typical wavefunction is not exponentially localized 68], while the logarithm of the conductance is not a selfaveraging random variable [73], as would otherwise occur on both sides of the mobility edge . A weaker divergence of the density of states on approaching the band center survives in the two-dimensional chiral-orthogonal and chiral-unitary symmetry classes [24, 31, 80,81, This divergence is captured by effective field theories for weak disorder that include two-dimensional NLSMs with compact, noncompact, or supersymmetric target manifolds, respectively. These two-dimensional NLSMs can be regularized by two-dimensional random network models [49]. In turn, the latter can be mapped onto spin-Hubbard-like chains, which, however, have been intractable to this date. One of the reasons is that a straightforward generalization of the methods of Refs. [52,53,54,55,56,57,58,60,77, 78,79] leads to spaces of states on the sites of the chain that are not irreducible representations of the corresponding Lie superalgebra. The structure of these spaces from the point of view of the supersymmetry of the underlying physical problem is a so far unresolved question.

A NLSM approach to the model studied by Dyson is not possible as there is no diffusive regime in one dimension. This difficulty can be remedied by considering thick quantum wires, which are also often referred to as quasi-one-dimensional wires. A thick quantum wire with a large number of transverse channels $N \gg 1$ appears in the limit where both the bare conductance and the mean free path $\ell$ scale with $N$. This limiting procedure justifies the derivation of a one-dimensional NLSM with any one of the 10 classical symmetric spaces as the target manifold. In particular, one can derive a one-dimensional NLSM with the target manifold corresponding to the chiralunitary symmetry class. In the same way as a two-dimensional NLSM can be regularized by a spin chain, a one-dimensional NLSM can be regularized by a zero-dimensional spin-Hubbardlike model with one or two sites. Solving such a spin-Hubbard-like model is a reasonable first step towards understanding the critical properties of the two-dimensional models in the chiralunitary symmetry class.

The purpose of this paper is to calculate the density of states in the close vicinity of the band center of a quantum wire belonging to the chiral unitary symmetry class for an arbitrary number $N$ of channels by using a representation of the problem in terms of an effective zero-dimensional "superspin" model. As we have pointed out earlier, in this case the space of states is not obtained from irreducible representations of a superalgebra. However, the Hamiltonian of the effective model can be written as a bilinear form in the generators of a superalgebra, and it is this construction that we refer to as "the superspin approach". The density of states has been obtained by the superspin approach only for $N=1[77,78,79]$.

The density of states of quasi-one-dimensional wires with arbitrary $N$ in the chiral symmetry classes has been obtained using an entirely different approach 82, 83, based on the DorokhovMello-Pereyra-Kumar (DMPK) equation. This is an equation for the joint probability distribution of the Lyapunov exponents that are related to the eigenvalues of the transfer matrix [84 along the wire viewed as a multi-channel scatterer. There it was shown that the density of states depends sensitively on the parity of the number of channels $N$. When $N$ is odd, the Dyson singularity is recovered. When $N$ is even, the density of states is controlled by random matrix theory up to multiplicative logarithmic corrections. This paper is devoted to uncovering this parity effect within the superspin formalism applied to the chiral-unitary symmetry class, the simplest among all 10 symmetry classes from the point of view of the DMPK equation [82, 85,. Whereas the approach based on the DMPK equation is geometric [86,87, the method to be presented below is algebraic. However, the DMPK equation is limited to quasi-one-dimensional wires, while our hope is that the algebraic insights gained in this work can be of use to solving spin-Hubbard-like 
models related to two-dimensional NLSMs in the chiral symmetry classes.

The band center of a superconducting quantum wire with broken spin-rotation symmetry is another example of a mobility edge separating two insulating phases [83, 88]. Remarkably, the density of states displays the same Dyson singularity as a chiral quantum wire with an odd number of channels [83,88]. This suggests a deep connection between the corresponding symmetric spaces that has been partially explored from a geometric point of view in Refs. [89.90]. Hereto we hope that the algebraic method used in this paper could shed some light on this connection.

As our computation of the density of states for a quasi-one-dimensional wire in the chiralunitary symmetry class is quite involved, we now summarize the main steps leading to the dependence

$$
\nu(\varepsilon) \sim \begin{cases}(\varepsilon \tau)^{-1}\left|\ln ^{-3}(\varepsilon \tau)\right|, & N \text { odd, } \\ (\varepsilon \tau)|\ln (\varepsilon \tau)|, & N \text { even, }\end{cases}
$$

of the density of states $\nu(\varepsilon)$ at the positive energy $\varepsilon$ measured relative to the band center whereby $0<\varepsilon \tau \ll 1$ with the mean scattering time $\tau$ of order $N^{2} \ell / v_{F}\left(\ell\right.$ is the mean free path and $v_{F}$ the Fermi velocity at the band center without disorder). The divergence of the density of states when $N$ is odd is nothing but the Dyson divergence for the single chain random hopping problem. For even $N$, there is a multiplicative logarithmic correction to the power law predicted by random matrix theory.

In Sec. 2. we define a quasi-one-dimensional random hopping model on the square lattice of length $L=M a$ and width $N a$ with $a$ being the lattice spacing and $L \gg N a$. We also provide a supersymmetric representation for the global density of states. In Sec. 月, the density of states in the thermodynamic limit $L \rightarrow \infty, L \gg N a$, is recast as a certain expectation value in the ground state of an operator $H$ acting on the direct product of a fermionic and a bosonic Fock spaces with a positive definite scalar product. The operator $H$ is an infinitesimal version of the transfer matrix along the wire written in second quantized language, and, therefore, we call $H$ the (effective) Hamiltonian, even though it is not Hermitian so that we need to distinguish its right and left eigenstates. Hamiltonian $H$ is quartic in terms of the fermionic and bosonic operators spanning the Fock space on which it acts. Moreover, Hamiltonian $H$, although not Hermitian, possesses a certain degree of supersymmetry, which allows us to construct its ground sate and thus to compute the density of states of the underlying quasi-one-dimensional wire. Finally, Hamiltonian $H$ depends parametrically on the energy scale $\varepsilon$ at which the density of states of the wire is to be evaluated and on the energy scale $1 / \tau$ characterizing the strength of the static disorder in the wire. The construction of the ground state of $H$ is done in Sec. I, where it is expanded in terms of certain basis states and where we find a recursion relation for the expansion coefficients. We do not solve this recursion relation exactly, but we solve it and calculate the corresponding density of states approximately close to the band center $\varepsilon \tau \ll 1$. The approximate solution is first presented in Sec. F for $N=1$ and $N=2$, and then for arbitrary even or odd $N$ in Sec. 6. We summarize the important steps of our derivation and make a comparison with the DMPK derivation in Sec. 7.

\section{The quasi-one-dimensional random hopping model}

In this paper we reconsider the density of states for a single quantum particle in a thick quantum wire. We start with a quasi-one-dimensional tight-binding system of spinless fermions with 
nearest-neighbor and next-nearest-neighbor random hopping matrix elements. On the square lattice the tight-binding Hamiltonian for $N$ coupled random hopping chains takes the form

$$
\mathcal{H}=-\sum_{i, j=1}^{N} \sum_{n=1}^{M}\left(t_{n ; i j} c_{n, i}^{\dagger} c_{n+1, j}+t_{n ; i j}^{\prime} c_{n, i}^{\dagger} c_{n+2, j}+\text { h. c. }\right) \text {. }
$$

Here, the indices $i$ and $j$ label the $N$ chains while $n$ labels the rows of sites in the direction along the wire. We impose the periodic boundary conditions along this direction, so that $M+1 \equiv 1$, etc. The spinless fermions are represented by operators that satisfy canonical anticommutation relations. The nearest-neighbor hopping strength $t_{n ; i j}$ consists of a large uniform part $t \delta_{i j}$ and a small random piece $\delta t_{n ; i j}$, whereas the next-nearest-hopping strength $t_{n ; i j}^{\prime}$ is assumed to be purely random and small. That is, the chains are only weakly coupled by small random hopping amplitudes. Perpendicular to the chains there is no notion of distance, as every chain is coupled with equal strength to every other one. We shall consider the quasi-one-dimensional limit, where the length $L$ of the disordered wire is much larger than its width $N a$, with $a$ the lattice constant.

For vanishing next-nearest-neighbor hopping, $t_{n ; i j}^{\prime}=0$, the Hamiltonian (2.1) reduces to the chiral random hopping model. This model is special because its energy eigenvalue spectrum exhibits a symmetry under charge conjugation, i.e., the Hamiltonian changes sign under the unitary transformation

$$
\left(c_{n, i}, c_{n, i}^{\dagger}\right) \rightarrow(-1)^{n}\left(c_{n, i}, c_{n, i}^{\dagger}\right)
$$

Hence, the eigenvalue spectrum of the chiral random hopping model has a reflection symmetry around the band center. As we shall see below, because of this extra symmetry the states near zero energy have localization properties that are dramatically different from those of the states in other parts of the spectrum.

Under the assumption of weak disorder,

$$
\delta t_{n ; i j}, t_{n ; i j}^{\prime} \ll t
$$

it is legitimate to take the continuum limit in the direction along the chains. In the absence of disorder the ground state of each chain consists of a filled Fermi sea bounded by two distinct Fermi points. The low energy excitations, the plane waves with momenta near these two points, are commonly called left- and right-movers, respectively. Linearizing the spectrum about the two Fermi points yields a kinetic energy that is a first-order differential operator. We thus model the dynamics of the single quantum particle in a quasi-one-dimensional geometry by

$$
\begin{aligned}
& \mathcal{H}_{\mathrm{c}}=\int_{0}^{L} d x \psi^{\dagger}(x) h_{\mathrm{c}}(x) \psi(x), \\
& h_{\mathrm{c}}(x)=-\mathrm{i} \sigma_{3} \partial_{x}+\sigma_{0} v_{0}(x)+\sigma_{1} v_{1}(x)+\sigma_{2} v_{2}(x)+\sigma_{3} v_{3}(x),
\end{aligned}
$$

where we choose our units such that $\hbar$ and the Fermi velocity $v_{F}$ are one. The spinors $\psi(x)$ are $2 N$-component vectors, $v_{\mu}(x)(\mu=0,1,2,3)$ are $N \times N$ random Hermitian matrices, and $\sigma_{\mu}$ denote the three Pauli matrices and the $2 \times 2$ unit matrix acting on the left-right-mover degrees of freedom. Due to gauge invariance the potential $v_{3}(x)$ can be chosen to be zero in a system with open boundary conditions. In the continuum language the chiral symmetry is implemented by the interchange of left and right movers and is represented by

$$
\sigma_{1} h_{\mathrm{c}}(x) \sigma_{1}=-h_{\mathrm{c}}(x)
$$

Chiral symmetry is thus only satisfied if the random matrices $v_{0}$ and $v_{1}$ vanish, i.e., a chiral symmetric disorder potential is here necessarily off-diagonal with respect to the left-right-mover 
degrees of freedom. In the presence of time-reversal symmetry, $\sigma_{1} h_{\mathrm{c}}^{*} \sigma_{1}=h_{\mathrm{c}}$, the disorder potentials satisfy the additional symmetry constraints

$$
v_{0 ; i j}^{*}=v_{0 ; i j}, \quad v_{1 ; i j}^{*}=v_{1 ; i j}, \quad v_{2 ; i j}^{*}=v_{2 ; i j},
$$

where $i, j=1, \cdots, N$. The disorder potentials $v_{\mu}$ are assumed to be independent and Gaussian distributed with zero means and and with variances

$$
\left[v_{\mu ; i j}(x) v_{\mu ; k l}^{*}\left(x^{\prime}\right)\right]_{\mathrm{av}}=2 g_{\mu} \delta\left(x-x^{\prime}\right)\left[\delta_{i k} \delta_{j l}+(2 / \beta-1) \delta_{i l} \delta_{j k}\right],
$$

respectively, where $\mu=0,1,2$ and the chain index $i, j, k, l=1, \cdots, N$. The disorder strength is denoted by $g_{\mu}$ and $\beta$ is the Dyson index with $\beta=1(2)$ in the presence (absence) of time-reversal symmetry.

We want to compute the Green function of the Hamiltonian (2.4) in order to obtain its density of states at the energy $\varepsilon$, which we assume to be positive, $\varepsilon>0$, without loss of generality. The two-point single-particle Green function is given by

$$
G\left(x, i, \alpha \mid x^{\prime}, i^{\prime}, \alpha^{\prime} ; \varepsilon+\mathrm{i} \eta\right)=\left\langle x, i, \alpha\left|\frac{1}{h_{\mathrm{c}}-\varepsilon-\mathrm{i} \eta}\right| x^{\prime}, i^{\prime}, \alpha^{\prime}\right\rangle,
$$

where $\alpha, \alpha^{\prime}=L, R$ are left-right-mover indices, $i, i^{\prime}=1, \cdots, N$ are channel indices, the infinitesimal regulator $\eta$ is strictly positive, $\eta>0$, and $|x, i, \alpha\rangle$ denotes a position eigenstate. The global density of states $\nu(\varepsilon)$ for a given realization of the disorder is then obtained from

$$
\nu(\varepsilon)=\lim _{\eta \rightarrow 0} \frac{1}{L} \int_{0}^{L} d x \frac{1}{N} \sum_{i=1}^{N} \sum_{\alpha=L, R} \frac{1}{\pi} \operatorname{Im} G(x, i, \alpha \mid x, i, \alpha ; \varepsilon+\mathrm{i} \eta) .
$$

It is convenient to introduce the notation

$$
\mathrm{i} \omega:=\varepsilon+\mathrm{i} \eta \Longleftrightarrow \omega:=-\mathrm{i} \varepsilon+\eta .
$$

The Green function 2.8 is an analytic function of $i \omega$ in the upper part of the complex plane for any realization of the disorder. By analytical continuation of $\varepsilon>0$ to the upper imaginary axis, $i \omega$ is strictly imaginary ( $\omega$ becomes strictly positive). If so, one has to analytically continue $\omega$ to the lower imaginary axis in order to obtain the density of states 2.9. Translation invariance is restored in the disorder average of the Green function, i.e., one can define

$$
G\left(x-x^{\prime} ; \mathrm{i} \omega\right)=\frac{1}{N}\left[\sum_{i=1}^{N} \sum_{\alpha=L, R} G\left(x, i, \alpha \mid x^{\prime}, i, \alpha ; \mathrm{i} \omega\right)\right]_{\mathrm{av}},
$$

where the square brackets on the right-hand side denote disorder averaging. Correspondingly, the mean global density of states is

$$
[\nu(\varepsilon)]_{\mathrm{av}}=\frac{1}{L} \int_{0}^{L} d x \lim _{\omega \rightarrow-\mathrm{i} \varepsilon} \lim _{x^{\prime} \rightarrow x} \frac{1}{\pi} \operatorname{Im} G\left(x-x^{\prime} ; \mathrm{i} \omega\right) .
$$

The global density of states becomes self-averaging in the thermodynamic limit $L \rightarrow \infty$, so that the brackets on the left-hand side of Eq. (2.12) can be omitted.

The single-particle Green functions (2.8) or 2.11) can be represented by functional path integrals as long as $i \omega$ belongs to the upper part of the complex plane. Unless specified, we assume that $\omega>0$. To perform the disorder average we make use of supersymmetry, so that 


$$
G\left(x-x^{\prime} ; \mathrm{i} \omega\right)=\frac{\mathrm{i}}{N}\left[\frac{1}{Z} \sum_{i=1}^{N} \sum_{\alpha=L, R} \int D \psi^{*} D \psi D \xi^{*} D \xi \psi_{i, \alpha}(x) \psi_{i, \alpha}^{*}\left(x^{\prime}\right) e^{-S}\right]_{\mathrm{av}},
$$

where the action

$$
S=\int_{0}^{L} d x \mathcal{L}
$$

is defined as the one-dimensions integral of the Lagrangian

$$
\mathcal{L}=\psi^{\dagger}\left(\mathrm{i} h_{\mathrm{c}}+\omega\right) \psi+\xi^{\dagger}\left(\mathrm{i} h_{\mathrm{c}}+\omega\right) \xi,
$$

and the partition function

$$
Z:=\int D \psi^{*} D \psi D \xi^{*} D \xi e^{-S} .
$$

We have introduced, for any channel index $i=1, \cdots, N$ and any left-right mover index $\alpha=$ $L, R$, the complex-valued integration variables $\xi_{i, \alpha}^{*}(x)$ and $\xi_{i, \alpha}(x)$, here related by complex conjugation, together with the pairs of independent Grassmann fields $\psi_{i, \alpha}^{*}(x)$ and $\psi_{i, \alpha}(x)$. In the functional integral formulation, the periodic boundary conditions imposed on the Hamiltonian (2.1) translate as follows: the bosonic fields obey periodic boundary conditions, but the fermionic fields obey antiperiodic boundary conditions in the $x$ direction. The assumption $\omega>0$ guarantees the convergence of the Gaussian integral over the complex-valued integration variables $\xi_{i, \alpha}^{*}(x)$ and $\xi_{i, \alpha}(x)$. The action and the measure of integration in the field theory 2.13a contains supersymmetries that rotate boson and fermion fields into each other. These symmetries guarantee that the corresponding partition function is unity in every disorder realization: $Z=1$, which simplifies the computation of averaged Green functions considerably. The ensemble average over the disorder configurations in Eq. 2.13a can be performed analytically by means of a cumulant expansion. With the Gaussian distribution (2.7), this gives the exact result

$$
G\left(x-x^{\prime} ; \mathrm{i} \omega\right)=\frac{\mathrm{i}}{N} \sum_{i=1}^{N} \sum_{\alpha=L, R} \int D \psi^{*} D \psi D \xi^{*} D \xi \psi_{i, \alpha}(x) \psi_{i, \alpha}^{*}\left(x^{\prime}\right) e^{-S_{\text {eff }},}
$$

where the effective action

$$
S_{\text {eff }}=\int_{0}^{L} d x \mathcal{L}_{\text {eff }}(x)
$$

is defined from the effective Lagrangian

$$
\begin{aligned}
\mathcal{L}_{\text {eff }}(x)= & \operatorname{Tr}\left(-\psi_{i, L}^{*} \partial_{x} \psi_{j, L}+\psi_{i, R}^{*} \partial_{x} \psi_{j, R}-\xi_{i, L}^{*} \partial_{x} \xi_{j, L}+\xi_{i, R}^{*} \partial_{x} \xi_{j, R}+\omega \mathcal{J}_{0 ; i j}\right)(x) \\
& +\sum_{\mu=0}^{2} g_{\mu} \operatorname{Tr}\left[\mathcal{\partial}_{\mu} \partial_{\mu}+(2 / \beta-1) \partial_{\mu} \partial_{\mu}^{T}\right](x) .
\end{aligned}
$$

Here, $\operatorname{Tr}(\cdots)$ refers to the trace over the channel index, we made use of the Hermiticity of the three random $N \times N$ matrix $v_{0,1,2}$, and we also introduced the $N \times N$ Hermitian matrices $\jmath_{0,1,2}$ and their transposed $\mathcal{J}_{0,1,2}^{T}$ through their matrix elements

$$
\begin{aligned}
& \mathcal{J}_{0 ; i j} \equiv \psi_{i, L}^{*} \psi_{j, L}+\psi_{i, R}^{*} \psi_{j, R}+\xi_{i, L}^{*} \xi_{j, L}+\xi_{i, R}^{*} \xi_{j, R}, \\
& \mathcal{J}_{1 ; i j} \equiv \psi_{i, R}^{*} \psi_{j, L}+\psi_{i, L}^{*} \psi_{j, R}+\xi_{i, R}^{*} \xi_{j, L}+\xi_{i, L}^{*} \xi_{j, R}, \\
& \mathcal{J}_{2 ; i j} \equiv \mathrm{i}\left(\psi_{i, R}^{*} \psi_{j, L}-\psi_{i, L}^{*} \psi_{j, R}+\xi_{i, R}^{*} \xi_{j, L}-\xi_{i, L}^{*} \xi_{j, R}\right),
\end{aligned}
$$


with $i, j=1, \cdots, N$.

We are going to demonstrate how we can use Feynman's transfer matrix method to trade the one-dimensional field theory encoded by Eqs. $(2.14 \mathrm{a}),(2.14 \mathrm{~b})$, and $(2.14 \mathrm{c})$ with $\omega>0$ for a zero-dimensional Schrödinger equation with a non-Hermitian superspin Hamiltonian. We will show that the non-Hermitian Hamiltonian possesses real eigenvalues and has manifestly unitary "imaginary-time evolution". We will also show how the density of states (2.12) can be extracted from an expectation value in the nondegenerate ground state annihilated by this non-Hermitian Hamiltonian.

\section{Mapping to a transfer Hamiltonian}

To perform the mapping onto a superspin Hamiltonian, we start from the classical Hamiltonian

$$
\mathcal{L}(p, q)-\sum_{i=1}^{N} \sum_{\operatorname{deg}=0,1} \sum_{\alpha=L, R} p_{i, \mathrm{deg}, \alpha} \dot{q}_{i, \mathrm{deg}, \alpha},
$$

that can be read off from the Lagrangian (2.13c) once $4 N$ coordinates

$$
q_{i, \mathrm{deg}, \alpha}, \quad i=1, \cdots, N, \quad \operatorname{deg}=0,1, \quad \alpha=1,2,
$$

have been chosen and by use of the $4 N$ momenta

$$
p_{i, \alpha}:=\frac{\delta \mathcal{L}}{\delta \dot{q}_{i, \alpha}}, \quad \dot{q}_{i, \alpha} \equiv \partial_{x} q_{i, \alpha}, \quad i=1, \cdots, N, \quad \operatorname{deg}=0,1, \quad \alpha=1,2,
$$

whereby $\delta / \delta \dot{q}_{i, \alpha}$ denotes the right derivative. We are thus interpreting $x$ as the imaginary time. There is considerable freedom in the choice of the $q$ 's and hence of the $p$ 's. The most obvious choice is

$$
q_{i} \equiv\left(q_{i, 0}, q_{i, 1}\right), \quad q_{i, 0}=\left(\xi_{i, L}, \xi_{i, R}\right), \quad q_{i, 1}=\left(\psi_{i, L}, \psi_{i, R}\right),
$$

for which the momenta are

$$
p_{i} \equiv\left(p_{i, 0}, p_{i, 1}\right), \quad p_{i, 0}=\left(-\xi_{i, L}^{*}, \xi_{i, R}^{*}\right), \quad p_{i, 1}=\left(-\psi_{i, L}^{*}, \psi_{i, R}^{*}\right),
$$

with the channel index running over $i=1, \cdots, N$. With this choice,

$$
\mathcal{L}=p \cdot \dot{q}+\operatorname{Tr}\left(\omega \mathcal{J}_{0}+\mathrm{i} v_{0}^{*} \mathcal{J}_{0}+\mathrm{i} v_{1}^{*} \mathcal{J}_{1}+\mathrm{i} v_{2}^{*} \mathcal{J}_{2}\right),
$$

where we have introduced the short-hand notation

$$
p \cdot \dot{q} \equiv \sum_{i=1}^{N} \sum_{\operatorname{deg}=0,1} \sum_{\alpha=L, R} p_{i, \mathrm{deg}, \alpha} \dot{q}_{i, \mathrm{deg}, \alpha}
$$

and, for any $i, j=1, \cdots, N$, Eq. 2.14d now reads

$$
\begin{aligned}
& \mathfrak{J}_{0 ; i j} \equiv-p_{i, 1, L} q_{j, 1, L}+p_{i, 1, R} q_{j, 1, R}-p_{i, 0, L} q_{j, 0, L}+p_{i, 0, R} q_{j, 0, R}, \\
& \mathfrak{J}_{1 ; i j} \equiv+p_{i, 1, R} q_{j, 1, L}-p_{i, 1, L} q_{j, 1, R}+p_{i, 0, R} q_{j, 0, L}-p_{i, 0, L} q_{j, 0, R}, \\
& \mathfrak{J}_{2 ; i j} \equiv \mathrm{i}\left(p_{i, 1, R} q_{j, 1, L}+p_{i, 1, L} q_{j, 1, R}+p_{i, 0, R} q_{j, 0, L}+p_{i, 0, L} q_{j, 0, R}\right) .
\end{aligned}
$$

Observe that the bilinear form $\mathcal{J}_{0} \equiv\left(\mathcal{J}_{0 ; i j}\right)$ has the undesirable property that it is not positive definite. Hence, the quantum operator corresponding to Eq. (3.1) is seemingly ill defined as it 
has an unbounded spectrum of eigenvalues from below upon quantization by which the classical $p$ 's and $q$ 's are replaced by operators that obey the canonical supercommutation relations

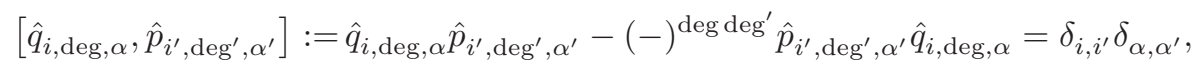

for any $i, i^{\prime}=1, \cdots, N$, deg, $\operatorname{deg}^{\prime}=0,1$, and $\alpha, \alpha^{\prime}=L, R$. To cure this difficulty it is convenient to redefine the vacuum in the fermionic sector of the theory to be the Dirac sea. However, by the spin-statistics theorem, this medicine is inoperative in the bosonic sector unless one is willing to either give up Hermiticity [78] or the positive definite scalar product of the Fock space on which the quantum operator corresponding to Eq. (3.1) is defined $[79$. In this paper, we choose the former route, so our quantum Hamiltonian will act on a space with a positive-definite scalar product, but will not be self-adjoint with respect to this product. In this setting, one will have to distinguish between the right and left eigenstates of the Hamiltonian.

For example, consider the quantum theory defined by the assignments

$$
\begin{aligned}
& \psi_{i, R}^{*} \rightarrow f_{i}^{\dagger}, \quad \psi_{i, R} \rightarrow f_{i}, \quad \xi_{i, R}^{*} \rightarrow b_{i}^{\dagger}, \quad \xi_{i, R} \rightarrow b_{i}, \\
& \psi_{i, L}^{*} \rightarrow \bar{f}_{i}, \quad \psi_{i, L} \rightarrow-\bar{f}_{i}^{\dagger}, \quad \xi_{i, L}^{*} \rightarrow \bar{b}_{i}, \quad \xi_{i, L} \rightarrow \bar{b}_{i}^{\dagger},
\end{aligned}
$$

with the only nonvanishing supercommutators

$$
\left\{f_{i}, f_{j}^{\dagger}\right\}=\left\{\bar{f}_{i}, \bar{f}_{j}^{\dagger}\right\}=\left[b_{i}, b_{j}^{\dagger}\right]=\left[\bar{b}_{i}, \bar{b}_{j}^{\dagger}\right]=\delta_{i j}, \quad i, j=1, \cdots, N .
$$

The corresponding Fock space $\mathfrak{F}_{\mathrm{R}}$ is obtained by action of the creation operators on the vacuum state, i.e., the state $|0\rangle$ that is annihilated by the right action of $f_{i}, \bar{f}_{i}, b_{i}, \bar{b}_{i}$ with $i=1,2, \cdots, N$, i.e., $\mathfrak{F}_{\mathrm{R}}$ is the linear span of the product states

$$
\prod_{i=1}^{N}\left(f_{i}^{\dagger}\right)^{n_{f_{i}}}\left(\bar{f}_{i}^{\dagger}\right)^{n_{\bar{f}_{i}}}\left(b_{i}^{\dagger}\right)^{n_{b_{i}}}\left(\bar{b}_{i}^{\dagger}\right)^{n_{\bar{b}_{i}}}|0\rangle,
$$

where

$$
n_{f_{i}}, n_{\bar{f}_{i}}=0,1, \quad n_{b_{i}}, n_{\bar{b}_{i}}=0,1,2, \cdots .
$$

We assume that the vacuum state is normalized to one,

$$
\langle 0 \mid 0\rangle=1,
$$

whereby the dual vacuum state $\langle 0|$ is the state annihilated by the left action of $f_{i}^{\dagger}, \bar{f}_{i}^{\dagger}, b_{i}^{\dagger}, \bar{b}_{i}^{\dagger}$ with $i=1,2, \cdots, N$. The identification (3.4) leads us to define the coherent states

$$
\begin{aligned}
& \left|\psi_{R}\right\rangle_{\mathrm{R}}=e^{-\psi_{i, R} f_{i}^{\dagger}}|0\rangle,{ }_{\mathrm{L}}\left\langle\psi_{R}\left|=\left\langle 0\left|e^{-\psi_{i, R}^{*} f_{i}},\right| \xi_{R}\right\rangle_{\mathrm{R}}=e^{+\xi_{i, R} b_{i}^{\dagger}}\right| 0\right\rangle,{ }_{\mathrm{L}}\left\langle\xi_{R}\right|=\langle 0| e^{+\xi_{i, R}^{*} b_{i}}, \\
& \left|\psi_{L}\right\rangle_{\mathrm{R}}=e^{-\psi_{i, L}^{*} \bar{f}_{i}^{\dagger}}|0\rangle,{ }_{\mathrm{L}}\left\langle\psi_{L}\left|=\left\langle 0\left|e^{+\psi_{i, L} \bar{f}_{i}},\right| \xi_{L}\right\rangle_{\mathrm{R}}=e^{+\xi_{i, L}^{*} \bar{b}_{i}^{\dagger}}\right| 0\right\rangle,{ }_{\mathrm{L}}\left\langle\xi_{L}\right|=\langle 0| e^{+\xi_{i, L} \bar{b}_{i}},
\end{aligned}
$$

where summation over $i=1, \cdots, N$ is implied, with the overlaps

$$
\begin{array}{ll}
{ }_{\mathrm{L}}\left\langle\psi_{R} \mid \psi_{R}^{\prime}\right\rangle_{\mathrm{R}}=e^{+\psi_{i, R}^{*} \psi_{i, R}^{\prime},}, & { }_{\mathrm{L}}\left\langle\xi_{R} \mid \xi_{R}^{\prime}\right\rangle_{\mathrm{R}}=e^{+\xi_{i, R}^{*} \xi_{i, R}^{\prime},} \\
{ }_{\mathrm{L}}\left\langle\psi_{L} \mid \psi_{L}^{\prime}\right\rangle_{\mathrm{R}}=e^{-\psi_{i, L} \psi_{i, L}^{\prime *}}, & { }_{\mathrm{L}}\left\langle\xi_{L} \mid \xi_{L}^{\prime}\right\rangle_{\mathrm{R}}=e^{+\xi_{i, L} \xi_{i, L}^{\prime *}} .
\end{array}
$$

We observe that $\left|\psi_{L}\right\rangle_{\mathrm{R}}$ and ${ }_{\mathrm{L}}\left\langle\psi_{L}\right|$ are not related by the "conventional" adjoint operation $(\cdots)^{\dagger}$. Instead, the right coherent states $\left|\psi_{L}\right\rangle_{\mathrm{R}}$ can be mapped onto the left coherent states $\left|\psi_{L}\right\rangle_{\mathrm{L}}$ by a unitary transformation $U$,

$$
\left|\psi_{L}\right\rangle_{\mathrm{L}}=\left(_{\mathrm{L}}\left\langle\psi_{L}\right|\right)^{\dagger}=U\left|\psi_{L}\right\rangle_{\mathrm{R}}, \quad U:=\exp \left(\mathrm{i} \pi \bar{f}_{i}^{\dagger} \bar{f}_{i}\right) .
$$


Due to this fact, it is necessary to distinguish between the right Fock space $\mathfrak{F}_{\mathrm{R}}$ and its dual, the left Fock space $\mathfrak{F}_{\mathrm{L}}^{*}$, that are related by the unitary transformation $(3.7)$. That is, for a given basis $\left|\Psi_{m}\right\rangle_{\mathrm{R}}$ of the right Fock space $\mathfrak{F}_{R}$, we define the corresponding basis $\left|\Psi_{m}\right\rangle_{\mathrm{L}}$ of the left Fock space $\mathfrak{F}_{\mathrm{L}}$ by acting with $U$ on the right basis, i.e., $\left|\Psi_{m}\right\rangle_{\mathrm{L}}=U\left|\Psi_{m}\right\rangle_{\mathrm{R}}$. The dual Fock space $\mathfrak{F}_{\mathrm{L}}^{*}$ is then the linear span of the basis ${ }_{L}\left\langle\Psi_{m}\right| \equiv\left(\left|\Psi_{m}\right\rangle_{L}\right)^{\dagger}$. Similarly, we will need to distinguish between left and right eigenstates of the transfer Hamiltonian $H$.

Equipped with the definition (3.6) for the coherent states, we are now ready to derive the effective transfer Hamiltonian. It is given by replacing $\mathcal{L}$ in Eq. (3.1a) with $\mathcal{L}_{\text {eff }}$ from Eq. (2.14c). After disorder averaging, the transfer Hamiltonian reads

$$
\begin{aligned}
& H=H_{\omega}+H_{\mathcal{V}}, \\
& H_{\omega}=\omega \operatorname{Tr} J_{0}=\omega\left(N_{f}+N_{\bar{f}}+N_{b}+N_{\bar{b}}\right), \\
& H_{\mathcal{V}}=\sum_{\mu=0}^{2} g_{\mu} \operatorname{Tr}\left[J_{\mu} J_{\mu}+(2 / \beta-1) J_{\mu} J_{\mu}^{\mathrm{T}}\right],
\end{aligned}
$$

with the number operators and disorder-induced operators defined by

$$
N_{b}:=\sum_{i=1}^{N} b_{i}^{\dagger} b_{i}, \quad N_{\bar{b}}:=\sum_{i=1}^{N} \bar{b}_{i}^{\dagger} \bar{b}_{i}, \quad N_{f}:=\sum_{i=1}^{N} f_{i}^{\dagger} f_{i}, \quad N_{\bar{f}}:=\sum_{i=1}^{N} \bar{f}_{i}^{\dagger} \bar{f}_{i},
$$

and

$$
\begin{aligned}
J_{0 ; i j} & :=+f_{i}^{\dagger} f_{j}-\bar{f}_{i} \bar{f}_{j}^{\dagger}+b_{i}^{\dagger} b_{j}+\bar{b}_{i} \bar{b}_{j}^{\dagger}, \\
J_{1 ; i j} & :=-f_{i}^{\dagger} \bar{f}_{j}^{\dagger}+\bar{f}_{i} f_{j}+b_{i}^{\dagger} \bar{b}_{j}^{\dagger}+\bar{b}_{i} b_{j}, \\
J_{2 ; i j} & :=-\mathrm{i}\left(f_{i}^{\dagger} \bar{f}_{j}^{\dagger}+\bar{f}_{i} f_{j}-b_{i}^{\dagger} \bar{b}_{j}^{\dagger}+\bar{b}_{i} b_{j}\right),
\end{aligned}
$$

respectively. In as much as the right Fock space $\mathfrak{F}_{R}$ and its dual, the left Fock space $\mathfrak{F}_{\mathrm{L}}^{*}$, that are associated with $H$ are not related by the conventional adjoint operation, the transfer Hamiltonian $H$ itself is not invariant under the conventional adjoint operation. On the one hand, $J_{0, i j}^{\dagger}=J_{0, j i}$. On the other hand, because of the fermions, $J_{1, i j}^{\dagger} \neq J_{1, j i}$, and $J_{2, i j}^{\dagger} \neq J_{2, j i}$. Consequently,

$$
H_{v} \neq H_{v}^{\dagger} \text {. }
$$

Upon making the transition to the transfer Hamiltonian $H$ as encoded by Eq. (3.4) the partition function $2.13 \mathrm{~d}$ ) becomes

$$
Z=\sum_{m}{ }_{L}\left\langle\Psi_{m}\left|(-1)^{N_{F}} e^{-L H}\right| \Psi_{m}\right\rangle_{\mathrm{R}}, \quad N_{F}:=N_{f}+N_{\bar{f}},
$$

where $N_{F}$ is the total fermion number operator, and the sum runs over some basis of the Fock space pair $\left(\mathfrak{F}_{\mathrm{L}}, \mathfrak{F}_{\mathrm{R}}\right)$, with $\left|\Psi_{m}\right\rangle_{\mathrm{L}}=U\left|\Psi_{m}\right\rangle_{\mathrm{R}}$ for all $m$. The factor $(-1)^{N_{F}}$ is a consequence of the antiperiodic boundary conditions obeyed by the original Grassmann integration variables at the ends of the wire.

Finally, we note that for a single channel, $N=1$, alternative representations of Hamiltonian (3.8a) have been studied in Refs. [77], [78], and [91] as well as in connection with the random network problem introduced in Ref. [79]. We are now going to show that the eigenvalues of $H$ for the chiral unitary symmetry class are manifestly real and that the matrix elements of $\exp (-L H)$ between left and right eigenvectors of $H$ define transition amplitudes of a unitary evolution operator, in spite of the fact that $H$ is non-Hermitian. 


\subsection{Properties of the transfer Hamiltonian}

In this section and thereafter we shall limit ourselves to the chiral-unitary symmetry class defined by the conditions

$$
g_{0}=g_{1}=0, \quad \beta=2,
$$

in Eq. (2.7). Hamiltonian (3.8a) thus reduces to

$$
H=H_{\omega}+H_{2}, \quad H_{\omega}=\omega \operatorname{Tr} J_{0}, \quad H_{2}=g_{2} \operatorname{Tr} J_{2} J_{2},
$$

in the chiral-unitary symmetry class. The original supersymmetry of the action $(2.14 \mathrm{~b})$ is realized by the invariance of Hamiltonian (3.8a) under the interchanges

$$
\left(f_{i}^{\dagger}, f_{i},+\bar{f}_{i}^{\dagger}, \bar{f}_{i}\right) \leftrightarrow\left(b_{i}^{\dagger}, b_{i},-\bar{b}_{i}^{\dagger}, \bar{b}_{i}\right) \quad i=1, \cdots, N .
$$

In addition, for the chiral classes $\left(g_{0}=g_{1}=0\right), H_{2}$ is unchanged by the interchanges

$$
\left(f_{i}, f_{i}^{\dagger},+b_{i}, b_{i}^{\dagger}\right) \leftrightarrow\left(\bar{f}_{i}^{\dagger}, \bar{f}_{i},-\bar{b}_{i}^{\dagger}, \bar{b}_{i}\right), \quad i=1, \cdots, N,
$$

whereas $H_{\omega}$ changes its sign under this transformation. This symmetry has its origin in the chiral symmetry. We also note that $\mathrm{H}_{2}$ commutes with the eight generators of the Lie superalgebra $g l(1 \mid 1) \oplus g l(1 \mid 1)$, while $H_{\omega}$ commutes with the four generators of the diagonal sub-algebra $g l(1 \mid 1) \subset g l(1 \mid 1) \oplus g l(1 \mid 1)$ (see Appendix A).

The invariance of $H$ under the supersymmetric transformation (3.13) has two consequences. First, it implies that an eigenvalue $E_{l}$ of $H$ can only be nondegenerate if its right eigenvector belongs to a singlet, i.e., if the right eigenvector is annihilated by the supersymmetric transformation (3.13). Second, any nonvanishing eigenvalue $E_{l}$ must be at least twofold degenerate with a pair of right eigenstates that are simultaneous eigenstates of the total fermion occupation number $N_{F}$ that differ by an odd number of fermions. That is, all the eigenstates with nonvanishing eigenenergy $E_{l}$ can be grouped into supersymmetric multiplets of degenerate eigenstates. The only exception to this rule, is the nondegenerate ground state, a singlet state, with $E_{l}=0$. Since the transfer Hamiltonian $H$ is non-Hermitian we need to distinguish left eigenstates from right eigenstates. In order to do so, we define right eigenstates, $\left|\varphi_{l, \iota}\right\rangle_{\mathrm{R}} \in \mathfrak{F}_{\mathrm{R}}$, with eigenvalues $E_{l}$ by

$$
H\left|\varphi_{l, \iota}\right\rangle_{\mathrm{R}}=E_{l}\left|\varphi_{l, \iota}\right\rangle_{\mathrm{R}} \Longleftrightarrow{ }_{\mathrm{R}}\left\langle\varphi_{l, \iota}\right| H^{\dagger}={ }_{\mathrm{R}}\left\langle\varphi_{l, l}\right| E_{l}^{*} .
$$

and left eigenstates, ${ }_{\mathrm{L}}\left\langle\varphi_{l, l}\right| \in \mathfrak{F}_{\mathrm{L}}^{*}$, of $H$ with eigenvalues $E_{l}$ by

$$
{ }_{\mathrm{L}}\left\langle\varphi_{l, L}\left|H={ }_{\mathrm{L}}\left\langle\varphi_{l, L}\left|E_{l}^{*} \Longleftrightarrow H^{\dagger}\right| \varphi_{l, L}\right\rangle_{\mathrm{L}}=E_{l}\right| \varphi_{l, L}\right\rangle_{\mathrm{L}},
$$

where the index $\iota$ labels the different elements of the supersymmetric multiplet with eigenenergy $E_{l}$. Since the right and left eigenstates are elements of the Fock space $\mathfrak{F}_{\mathrm{R}}$ and $\mathfrak{F}_{\mathrm{L}}^{*}$, respectively, it is possible to map $\left|\varphi_{l, L}\right\rangle_{\mathrm{R}}$ onto $\left|\varphi_{l, L}\right\rangle_{\mathrm{L}}$ by use of the unitary transformation $U$ as defined in Eq. (3.7),

$$
\left|\varphi_{l, \iota}\right\rangle_{\mathrm{L}}=U\left|\varphi_{l, \iota}\right\rangle_{\mathrm{R}} \quad \Longleftrightarrow \quad{ }_{\mathrm{L}}\left\langle\varphi_{l, \iota}\right|={ }_{\mathrm{R}}\left\langle\varphi_{l, \iota}\right| U^{\dagger} .
$$

Conversely, the unitary transformation $U$ can be used to compute the adjoint of both $J_{2}$ and the transfer Hamiltonian $H$. Namely, we find that

$$
J_{2}^{\dagger}=U J_{2}^{T} U^{\dagger}, \quad H^{\dagger}=U H U^{\dagger},
$$

where $(\cdots)^{T}$ denotes the transpose operating on the $N$ scattering channels. 
As for the single channel problem of Ref. [77], we can now use the left and right eigenstates (3.15) to construct a normalized eigenbasis and the corresponding resolution of identity. Thereto, we argue that the left and right eigenstates of $H$ in any given supersymmetric multiplet with eigenenergy $E_{l}$ can be grouped into pairs $\left(\left|\varphi_{l, L}\right\rangle_{\mathrm{R} / \mathrm{L}},\left|\varphi_{l, \sigma[l]}\right\rangle_{\mathrm{R} / \mathrm{L}}\right)$ normalized by the condition

$$
{ }_{\mathrm{L}}\left\langle\varphi_{l, \iota} \mid \varphi_{l, \sigma[l]}\right\rangle_{\mathrm{R}}=1,
$$

and such that the left and right eigenstates form a well defined biorthogonal system with

$$
{ }_{\mathrm{L}}\left\langle\varphi_{l, \iota} \mid \varphi_{l^{\prime}, \iota^{\prime}}\right\rangle_{\mathrm{R}}=\delta_{l, l^{\prime}} \delta_{\sigma[\iota], \iota^{\prime}}
$$

for all energy eigenvalue indices $l, l^{\prime}$ and for all supersymmetric multiplet index $\iota, \iota^{\prime}$. Here, we have introduced the permutation function $\sigma[\iota]$ of the index $\iota$ that parametrizes the pairing of the eigenstates. The permutation function is involutive, i.e., it satisfies $\sigma^{2}=1$. The resolution of identity is then given by

$$
\sum_{l, \iota}\left|\varphi_{l, \sigma[\iota]}\right\rangle_{\mathrm{RL}}\left\langle\varphi_{l, \iota}\right|=1
$$

These properties of the eigenstates can now be used to show that the transfer Hamiltonian $H$ with its associated Fock space pair $\left(\mathfrak{F}_{\mathrm{L}}, \mathfrak{F}_{\mathrm{R}}\right)$ possesses a real eigenvalue spectrum, and that the operator $\exp (-L H)$ defines a unitary time evolution with respect to the Fock space pair $\left(\mathfrak{F}_{\mathrm{L}}, \mathfrak{F}_{\mathrm{R}}\right)$. First, for any given eigenenergy $E_{l}$ with left eigenvector $\left|\varphi_{l, \iota}\right\rangle_{\mathrm{L}}$ and right eigenvector $\left|\varphi_{l, \sigma[\iota]}\right\rangle_{\mathrm{R}}$ we find

$$
E_{l}^{*}={ }_{\mathrm{R}}\left\langle\varphi_{l, \sigma[\iota]}\left|H^{\dagger}\right| \varphi_{l, \iota}\right\rangle_{\mathrm{L}}={ }_{\mathrm{R}}\left\langle\varphi_{l, \sigma[\iota]}\left|U H U^{\dagger}\right| \varphi_{l, \iota}\right\rangle_{\mathrm{L}}={ }_{\mathrm{L}}\left\langle\varphi_{l, \sigma[\iota]}|H| \varphi_{l, \iota}\right\rangle_{\mathrm{R}}=E_{l},
$$

where we have used the mapping between left and right eigenstates $(3.16)$, the Hermitian adjoint of $H$ (3.17), and the normalization of the states 3.18). Second, the transition amplitude from the right energy eigenstate $\left|\varphi_{l, \iota}\right\rangle_{\mathrm{R}}$ to the left energy eigenstate $\left|\varphi_{l^{\prime}, \iota^{\prime}}\right\rangle_{\mathrm{L}}$ is defined by

$$
W_{l \iota, l^{\prime} \iota^{\prime}}(t):={ }_{\mathrm{L}}\left\langle\varphi_{l^{\prime}, \iota^{\prime}}\left|e^{-\mathrm{i} t H}\right| \varphi_{l, \iota}\right\rangle_{\mathrm{R}}
$$

after the analytic continuation $L \rightarrow \mathrm{it}$. Using Eqs. (3.15), Eqs. (3.18), and the involutive property of $\sigma[\iota]$, one verifies that, for all $t$,

$$
\begin{aligned}
\sum_{l^{\prime}, \iota^{\prime}}\left|W_{l \iota, l^{\prime} \iota^{\prime}}\right|^{2}(t) & =\sum_{l^{\prime}, \iota^{\prime}} \mathrm{R}\left\langle\varphi_{l, L}\left|e^{+\mathrm{i} t H^{\dagger}}\right| \varphi_{l^{\prime}, \iota^{\prime}}\right\rangle_{\mathrm{L} L}\left\langle\varphi_{l^{\prime}, \iota^{\prime}}\left|e^{-\mathrm{i} t H}\right| \varphi_{l, \iota}\right\rangle_{\mathrm{R}} \\
& =\sum_{l^{\prime}, \iota^{\prime}} \mathrm{R}\left\langle\varphi_{l, \iota}\left|e^{+\mathrm{i} t E_{l^{\prime}}}\right| \varphi_{l^{\prime}, \iota^{\prime}}\right\rangle_{\mathrm{L}} e^{-\mathrm{i} t E_{l}} \delta_{l^{\prime}, l} \delta_{\sigma\left[\iota^{\prime}\right], \iota} \\
& =1 .
\end{aligned}
$$

Hence, the expression (3.21) constitutes a well-defined transition amplitude, since it satisfies the condition of probability conservation associated to a unitary time evolution.

Summarizing, we have found that Eq. (3.17) implements the adjoint operation for the nonHermitian Hamiltonian $H$. The operator $U$ maps the right eigenstates from $\mathfrak{F}_{\mathrm{R}}$ into the dual space $\mathfrak{F}_{L}$ (i.e., the space of left eigenstates) in such a way that the transfer Hamiltonian becomes Hermitian within the Fock space pair $\left(\mathfrak{F}_{L}, \mathfrak{F}_{R}\right)$. That is, in order to reinstate unitarity of the evolution operator, $\exp (-\mathrm{i} t H)$, it is necessary to include the action of $U$ in the scalar product. 


\subsection{Quantum representation of the density of states}

We are going to give a quantum representation of the density of states 2.12 in the long wire limit $L \rightarrow \infty$ and show how the original supersymmetry and a well-defined density of states 2.12 imply that the spectrum of $H$ is positive definite.

We start by reexpressing the partition function (2.13d) [see also Eq. (3.10)] in terms of the eigenbasis 3.15)

$$
Z=\sum_{l, L}{ }_{L}\left\langle\varphi_{l, L}\left|(-1)^{N_{F}} e^{-L E_{l}}\right| \varphi_{l, L}\right\rangle_{\mathrm{R}}=1 .
$$

By the construction of Section 2, the supersymmetry implies that the partition function is exactly one whatever the length $L$ of the wire. In the transfer Hamiltonian representation of the partition function (3.23), this is born out by the fact that each supersymmetric eigenmultiplet of $H$ contains equal numbers of fermionic ( $N_{F}$ odd) and bosonic $\left(N_{F}\right.$ even) eigenstates, which thereby cancel in the supertrace due to the factor $(-1)^{N_{F}}$. Hence, the sum in Eq. (3.23) must then reduce to the expectation value in the zero-energy eigenenergy sector $E_{0}=0$ of the pair $\left(\mathfrak{F}_{L}, \mathfrak{F}_{R}\right)$. This sum then gives unity if and only if the eigenspace $E_{0}=0$ in $\left(\mathfrak{F}_{L}, \mathfrak{F}_{R}\right)$ is of dimension one. For the partition function to be independent of the wire length $L$, the spectrum of $H$ must contain a nondegenerate eigenenergy $E_{0}=0$.

In the quantum representation, the density of states $(2.12)$ is expressed by

$$
\begin{aligned}
\nu(\varepsilon) & =\lim _{\omega \rightarrow-\mathrm{i} \varepsilon} \lim _{L \rightarrow \infty} \pi^{-1} \operatorname{Re} \sum_{l, \iota}\left\langle\varphi_{l, \iota}\left|(-1)^{N_{F}}(\bar{B}-B) e^{-L H}\right| \varphi_{l, \iota}\right\rangle_{\mathrm{R}} \\
& =\lim _{\omega \rightarrow-\mathrm{i} \varepsilon} \lim _{L \rightarrow \infty} \pi^{-1} \operatorname{Re} \sum_{l, \iota}\left\langle\varphi_{l, l}\left|(-1)^{N_{F}}(Q-\bar{Q}) e^{-L H}\right| \varphi_{l, L}\right\rangle_{\mathrm{R}},
\end{aligned}
$$

where either

$$
B:=+\sum_{i=1}^{N} f_{i}^{\dagger} f_{i}-\frac{1}{2} N, \quad \bar{B}:=+\sum_{i=1}^{N} \bar{f}_{i} \bar{f}_{i}^{\dagger}-\frac{1}{2} N,
$$

or

$$
Q:=+\sum_{i=1}^{N} b_{i}^{\dagger} b_{i}+\frac{1}{2} N, \quad \bar{Q}:=-\sum_{i=1}^{N} \bar{b}_{i} \bar{b}_{i}^{\dagger}+\frac{1}{2} N .
$$

As the system becomes infinite in length, $L \rightarrow \infty$, any exponential term in Eq. (3.24), vanishes for all positive energy eigenstates. In order for the density of states to be well defined in the long wire limit, the eigenvalues of $H$ need to be positive. If so, the density of states in the limit $L \rightarrow \infty$ is dominated by the ground state expectation value with $E_{0}=0$

$$
\begin{aligned}
\nu(\varepsilon) & =\lim _{\omega \rightarrow-\mathrm{i} \varepsilon} \pi^{-1} \operatorname{Re}_{\mathrm{L}}\left\langle\varphi_{0}|(\bar{B}-B)| \varphi_{0}\right\rangle_{\mathrm{R}} \\
& =\lim _{\omega \rightarrow-\mathrm{i} \varepsilon} \pi^{-1} \operatorname{Re}_{\mathrm{L}}\left\langle\varphi_{0}|(Q-\bar{Q})| \varphi_{0}\right\rangle_{\mathrm{R}},
\end{aligned}
$$

where $\left|\varphi_{0}\right\rangle_{\mathrm{L}}$ and $\left|\varphi_{0}\right\rangle_{\mathrm{R}}$ denote the nondegenerate left and right ground state wave functions, respectively. In Eq. (3.25) we have dropped the factor $(-1)^{N_{F}}$ since the left and right ground state wave functions contain an even number of fermions, as we will explicitly verify in Section $\theta$. 


\section{Construction of the ground state sector}

In order to compute the density of states in the long wire limit (3.25), we need to make an appropriate Ansatz for the solutions $\left|\varphi_{0}\right\rangle_{\mathrm{R}}$ and $\left|\varphi_{0}\right\rangle_{\mathrm{L}}$ of the right and left Schrödinger equations

$$
H\left|\varphi_{0}\right\rangle_{\mathrm{R}}=\left(H_{\omega}+H_{2}\right)\left|\varphi_{0}\right\rangle_{\mathrm{R}}=0, \quad H^{\dagger}\left|\varphi_{0}\right\rangle_{\mathrm{L}}=\left(H_{\omega}^{\dagger}+H_{2}^{\dagger}\right)\left|\varphi_{0}\right\rangle_{\mathrm{L}}=0
$$

respectively. The left and right ground state wave functions are related by $\left|\varphi_{0}\right\rangle_{\mathrm{L}}=U\left|\varphi_{0}\right\rangle_{\mathrm{R}}$. As dictated by supersymmetry, the ground state wave function is nondegenerate and constitutes a supersymmetric singlet, i.e., it is annihilated by the supersymmetric transformation (3.13). Therefore, the ground state sector can be expanded in terms of states that transform as singlets under the supersymmetry (3.13). One such state is the vacuum $|0\rangle$. Other states that transform as singlets under the symmetry (3.13) can be generated by repeated action of $H$ on the vacuum $|0\rangle$. All the states that are generated by repeated action of $H$ on the vacuum $|0\rangle$ form a vector space, which we call the right ground state sector $\mathfrak{G}_{\mathrm{R}}$. The dual ground state sector $\mathfrak{G}_{\mathrm{L}}^{*}$ is generated by repeated action of $H^{\dagger}$ on the vacuum $\langle 0|$. Alternatively, $\mathfrak{G}_{\mathrm{L}}$ can be obtained by the application of the unitary transformation $U$ defined in Eq. (3.7) on the right ground state sector $\mathfrak{G}_{\mathrm{R}}$. In turn, $\mathfrak{G}_{\mathrm{L}}^{*}$ follows from $\mathfrak{G}_{\mathrm{L}}$ with the help of the adjoint operation. The ground state sectors are subsets of the corresponding Fock spaces, i.e., $\mathfrak{G}_{\mathrm{L}} \subsetneq \mathfrak{F}_{\mathrm{L}}$ and $\mathfrak{G}_{\mathrm{R}} \subsetneq \mathfrak{F}_{\mathrm{R}}$. We shall thus expand the right (left) ground state $\left|\varphi_{0}\right\rangle_{\mathrm{R}}\left(\left|\varphi_{0}\right\rangle_{\mathrm{L}}\right)$ of $H$ in a basis of the right (left) ground state sector $\mathfrak{G}_{\mathrm{R}}\left(\mathfrak{G}_{\mathrm{L}}\right)$.

Thereto we construct in what follows a right basis

$$
\left\{|m\rangle_{\mathrm{R}}^{(n)}\right\}_{m, n} \cup|0\rangle, \quad m, n=1,2, \cdots,
$$

and a left basis

$$
\{\stackrel{(n)}{\mathrm{L}}\langle m|\}_{m, n} \cup\langle 0|, \quad m, n=1,2, \cdots,
$$

of the ground state sectors $\mathfrak{G}_{\mathrm{R}}$ and $\mathfrak{G}_{\mathrm{L}}$, respectively. The left orthogonal set $|m\rangle_{\mathrm{L}}^{(n)}$ and the right orthogonal set $|m\rangle_{\mathrm{R}}^{(n)}$ are here generated with the help of the auxiliary orthogonal states

$$
\begin{aligned}
& |2 m+2,2 n ; \downarrow\rangle:=\left[\left(A_{-}\right)^{2 n}\left(D_{-}\right)^{2 m+2}-2 n S_{-} \bar{S}_{+}\left(A_{-}\right)^{2 n-1}\left(D_{-}\right)^{2 m+1}\right]|0\rangle, \\
& |2 m+1,2 n+1 ; \uparrow\rangle:=\left[\left(A_{-}\right)^{2 n+1}\left(D_{-}\right)^{2 m+1}+(2 m+1) S_{-} \bar{S}_{+}\left(A_{-}\right)^{2 n}\left(D_{-}\right)^{2 m}\right]|0\rangle, \\
& |2 m, 2 n+2 ; \uparrow\rangle:=\left[\left(A_{-}\right)^{2 n+2}\left(D_{-}\right)^{2 m}+2 m S_{-} \bar{S}_{+}\left(A_{-}\right)^{2 n+1}\left(D_{-}\right)^{2 m-1}\right]|0\rangle, \\
& |2 m+1,2 n+1 ; \downarrow\rangle:=\left[\left(A_{-}\right)^{2 n+1}\left(D_{-}\right)^{2 m+1}-(2 n+1) S_{-} \bar{S}_{+}\left(A_{-}\right)^{2 n}\left(D_{-}\right)^{2 m}\right]|0\rangle,
\end{aligned}
$$

defined by taking arbitrary integer powers of the raising operators

$$
A_{-}:=\sum_{a=1}^{N} f_{a}^{\dagger} \bar{f}_{a}^{\dagger}, \quad D_{-}:=\sum_{a=1}^{N} b_{a}^{\dagger} \bar{b}_{a}^{\dagger}, \quad S_{-}:=\sum_{a=1}^{N} f_{a}^{\dagger} \bar{b}_{a}^{\dagger}, \quad \bar{S}_{+}:=\sum_{a=1}^{N} b_{a}^{\dagger} \bar{f}_{a}^{\dagger},
$$

acting on $|0\rangle$. The basis sets $|m\rangle_{\mathrm{L}}^{(n)}$ and $|m\rangle_{\mathrm{R}}^{(n)}$ are countably infinite due to the presence of boson creation operators. The basis set $|m\rangle_{\mathrm{R}}^{(n)}$ is given by 

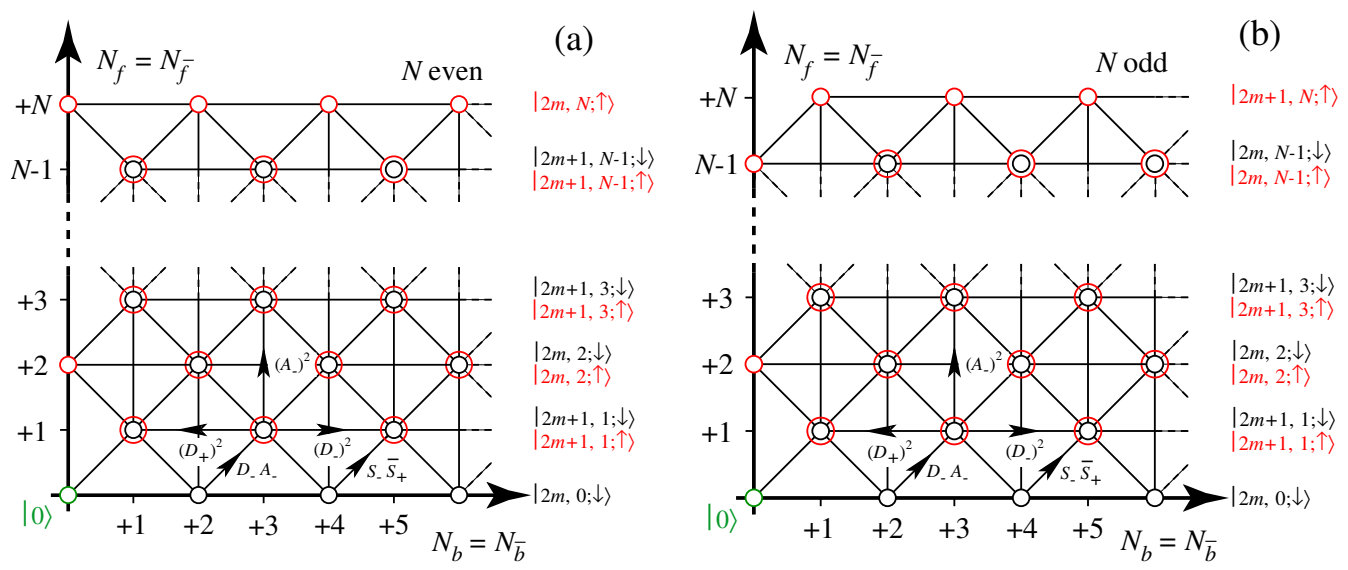

Fig. 1. (Color online) These weight diagrams depict states $4.3 \mathrm{a})$ in the $\left(N_{b}, N_{f}\right)$ plane. The left panel (a) displays the case of an even channel number $N$, whereas the right panel (b) shows the case of an odd channel number.

$$
\begin{aligned}
|m\rangle_{\mathrm{R}}^{(2 n+1)} & :=\frac{1}{\sqrt{2}} \frac{(N-2 n-1) !}{(N+2 m-1) !}(|2 m, 2 n ; \downarrow\rangle+|2 m-1,2 n+1 ; \uparrow\rangle), \\
|m\rangle_{\mathrm{R}}^{(2 n+2)}: & :=\frac{1}{\sqrt{2}} \frac{(N-2 n-2) !}{(N+2 m-2) !}(|2 m-2,2 n+2 ; \uparrow\rangle+|2 m-1,2 n+1 ; \downarrow\rangle),
\end{aligned}
$$

where $m=1,2,3, \cdots$. The basis $|m\rangle_{\mathrm{L}}^{(n)}$ is given by

$$
\begin{aligned}
|m\rangle_{\mathrm{L}}^{(2 n+1)} & :=\frac{1}{\sqrt{2}} \frac{(N-2 n-1) !}{(N+2 m-1) !}(|2 m, 2 n ; \downarrow\rangle-|2 m-1,2 n+1 ; \uparrow\rangle), \\
|m\rangle_{\mathrm{L}}^{(2 n+2)}: & :=\frac{1}{\sqrt{2}} \frac{(N-2 n-2) !}{(N+2 m-2) !}(|2 m-2,2 n+2 ; \uparrow\rangle-|2 m-1,2 n+1 ; \downarrow\rangle),
\end{aligned}
$$

where $m=1,2,3, \cdots$.

The states 4.3a are eigenstates of the operators $N_{f}, N_{\bar{f}}, N_{b}$, and $N_{\bar{b}}$ defined in Eq. (3.8b), whereby the eigenvalues of $N_{f}-N_{\bar{f}}$ and $N_{b}-N_{\bar{b}}$ are vanishing for all the states in (4.3a). It is therefore convenient to depict these states in the $\left(N_{b}, N_{f}\right)$-plane (see Fig. 1). As a consequence of Pauli's principle, the fermion number $N_{f}$ is restricted to a finite range. That is, any power larger than one of any of the fermionic operators $S_{-}$and $\bar{S}_{+}$vanishes, and for a given channel number $N$ we have

$$
\left(A_{-}\right)^{N+1}=0
$$

and

$$
\left(A_{-}\right)^{N} D_{-}=N S_{-} \bar{S}_{+}\left(A_{-}\right)^{N-1} \text {. }
$$

Condition (4.5) determines the range over which the index $n$ runs in Eqs. (4.3) and (4.4). We note that there is, as we shall see, a fundamental difference between even and odd channel numbers. For $N$ even the states with the highest fermion number $N_{f}$ are labeled by even boson numbers $N_{b}$, whereas for $N$ odd they are labeled by odd boson numbers $N_{b}$. The right and left bases (4.4) are related by the unitary transformation $U$ of Eq. (3.7), through

$$
|m\rangle_{\mathrm{L}}^{(n)}=U|m\rangle_{\mathrm{R}}^{(n)}, \quad m=1,2, \cdots, \quad n=1,2, \cdots, N,
$$

as it should be. The norms in the right sets (4.4a) are 


$$
\begin{gathered}
\quad{ }_{\mathrm{R}}^{(2 n+1)}\langle m \mid m\rangle_{\mathrm{R}}^{(2 n+1)}=(2 m+2 n)(2 m-1) !(2 n) ! \frac{(N-2 n-1) !}{(N+2 m-1) !}=: \mathcal{N}_{m, n}^{(1)}, \\
\underset{\mathrm{R}}{(2 n+2)}\langle m \mid m\rangle_{\mathrm{R}}^{(2 n+2)}=(2 m+2 n)(2 m-2) !(2 n+1) ! \frac{(N-2 n-2) !}{(N+2 m-2) !}=: \mathcal{N}_{m, n}^{(2)},
\end{gathered}
$$

with $m=1,2,3, \cdots$. It follows from 4.7 , that the norm for a given state in the left set is equal to the norm of the corresponding state in the right set. Moreover, the left and right sets 4.4 a) and (4.4b) are biorthogonal in the sense that

$$
{ }_{\mathrm{L}}^{(n)}\left\langle m \mid m^{\prime}\right\rangle_{\mathrm{R}}^{\left(n^{\prime}\right)}=0, \quad m, m^{\prime}=1,2, \cdots, \quad n, n^{\prime}=1,2, \cdots, N,
$$

as follows from Eq. (4.8) and the orthogonality of the states Eq. (4.3a).

Equipped with a basis for the right and left ground state sectors, we are now in a position to expand the right ground state

$$
\left|\varphi_{0}\right\rangle_{\mathrm{R}} \equiv\left({ }_{\mathrm{R}}\left\langle\varphi_{0}\right|\right)^{\dagger}=a^{(0)}|0\rangle+\sum_{n=1}^{N} \sum_{m=1}^{\infty} a_{m}^{(n)}|m\rangle_{\mathrm{R}}^{(n)},
$$

in terms of the basis Eq. 4.2a) and the left ground state

$$
\left|\varphi_{0}\right\rangle_{\mathrm{L}}=\left({ }_{\mathrm{L}}\left\langle\varphi_{0}\right|\right)^{\dagger}=a^{(0)}|0\rangle+\sum_{n=1}^{N} \sum_{m=1}^{\infty} a_{m}^{(n)}|m\rangle_{\mathrm{L}}^{(n)},
$$

in terms of the basis Eq. 4.2b). The coefficients of these two expansions are identical and are determined by solving the Schrödinger equations (4.1). The overlap between the expansions (4.10) for the right and left ground states follows form Eq. 4.9),

$$
{ }_{\mathrm{L}}\left\langle\varphi_{0} \mid \varphi_{0}\right\rangle_{\mathrm{R}}=\left|a^{(0)}\right|^{2} .
$$

Consequently, in order to normalize the ground state wave function, we set

$$
a^{(0)}=1
$$

from now on.

\subsection{Ground state Schrödinger equation}

Hamiltonian (3.12) depends on two energy scales: the (imaginary) energy $\omega$ at which the density of states in the thermodynamic limit is to be evaluated and the chiral disorder strength $g_{2}$ defined in Eq. 2.7.). Since the density of states in the thermodynamic limit is controlled solely by the right $\left|\varphi_{0}\right\rangle_{\mathrm{R}}$ and left $\left|\varphi_{0}\right\rangle_{\mathrm{L}}$ eigenstates annihilated by Hamiltonian (3.12), it must be a scaling function of the dimensionless variable

$$
\frac{\omega}{g_{2}} \rightarrow \omega
$$

provided we set $g_{2}$ to be unity, as we shall do from now on unless stated otherwise. The right (left) ground state $\left|\varphi_{0}\right\rangle_{\mathrm{R}}\left(\left|\varphi_{0}\right\rangle_{\mathrm{L}}\right)$ satisfies the eigenvalue equation (4.1). In the basis (4.2), the eigenvalue problem (4.1) yields the recursion relation for the coefficients $a_{m}^{(n)}$ of Eq. (4.10) given by 


$$
\begin{aligned}
2(2 m+2 n) \omega a_{m}^{(2 n+1)}= & -2(2 m+2 n)(2 m-1-2 n+N) a_{m}^{(2 n+1)} \\
& +2 m(2 m+1) a_{m+1}^{(2 n+1)} \\
& +(N+2 m-1)(N+2 m-2) a_{m-1}^{(2 n+1)} \\
& -(N-2 n)(N-2 n+1) a_{m}^{(2 n-1)} \\
& -(2 n+1)(2 n+2) a_{m}^{(2 n+3)},
\end{aligned}
$$

when $n=0,1, \cdots,[(N-1) / 2]$ and

$$
\begin{aligned}
2(2 m+2 n) \omega a_{m}^{(2 n+2)}= & -2(2 m+2 n)(2 m-3-2 n+N) a_{m}^{(2 n+2)} \\
& +2 m(2 m-1) a_{m+1}^{(2 n+2)} \\
& +(N+2 m-3)(N+2 m-2) a_{m-1}^{(2 n+2)} \\
& -(N-2 n)(N-2 n-1) a_{m}^{(2 n)} \\
& -(2 n+2)(2 n+3) a_{m}^{(2 n+4)},
\end{aligned}
$$

when $n=0,1, \cdots,[N / 2]-1$ and where $m=2,3,4, \cdots$. Here, it is understood that $a_{m}^{(n)}=0$ when $n \leq 0$ or $n>N$. The initial conditions are

$$
\begin{aligned}
& 4 \omega a_{1}^{(1)}=+\sqrt{2} N(N+1)-4(N+1) a_{1}^{(1)}+6 a_{2}^{(1)}-2 a_{1}^{(3)}, \\
& 4 \omega a_{1}^{(2)}=-\sqrt{2} N(N-1)-4(N-1) a_{1}^{(2)}+2 a_{2}^{(2)}-6 a_{1}^{(4)} .
\end{aligned}
$$

Equations (4.13) are a generalization to the case of an arbitrary number $N$ of channels of the recursion relations obtained by Refs. [77,78,91, when $N=1$. The rest of this paper is devoted to solving them and evaluating the density of states using Eq. (3.25).

\section{Evaluation of the density of states when $N=1$ and $N=2$}

Before we solve the recursion relations (4.13) for arbitrary $N$, we first specialize to the case $N=1$ and $N=2$ for pedagogical reasons. Only the case $N=1$ using the supersymmetric quantum representation (see Refs. [77, [78, 21]) was treated in the literature. The case $N=2$ was solved with the help of the DMPK equation in Refs. [82] and [83].

\subsection{Density of states for a single channel $N=1$}

For a single channel, $N=1$, the recursion relations Eq. (4.13) for the coefficients $a_{m}^{(1)}$ simplify to

$$
\begin{aligned}
2 \omega a_{1}^{(1)} & =+\sqrt{2}-4 a_{1}^{(1)}+3 a_{2}^{(1)}, \\
2 \omega a_{m+1}^{(1)} & =-2(2 m+2) a_{m+1}^{(1)}+(2 m+3) a_{m+2}^{(1)}+(2 m+1) a_{m}^{(1)},
\end{aligned}
$$

with $m=1,2,3, \cdots$. This recursion relation is identical to the one obtained by Gogolin $e t$ al. 171] by means of the Berezinskii diagram technique. Hence, one can identify the coefficients $a_{m}^{(1)}$ as the so-called right-hand side Berezinskii block. The recursion relation $(5.1 \mathrm{~b})$ can also be written in terms of the finite differences

$$
\Delta a_{m}^{(1)}:=a_{m+1}^{(1)}-a_{m}^{(1)}, \quad \Delta^{2} a_{m}^{(1)}:=a_{m+2}^{(1)}-2 a_{m+1}^{(1)}+a_{m}^{(1)} .
$$

That is, Eqs. (5.1b) and (5.2a) yield 


$$
2 \omega a_{m}^{(1)}=+(2 m+3) \Delta^{2} a_{m}^{(1)}+(2-2 \omega) \Delta a_{m}^{(1)},
$$

where $m=1,2,3 \cdots$.

The strategy to analyze equations $(5.1)$ in the limit of asymptotically small frequency, $\omega \ll 1$, is to derive two approximate solutions that are valid in two distinct asymptotic limits. For large $m \gg 1$, the variable $m$ can be taken as continuous. This reduces the recursion relation (5.1b) to a Bessel differential equation. For small $m \omega \ll 1$, we can neglect the $\omega$ term in Eq. (5.1b). With this approximation, it becomes possible to solve the resulting recursion relation exactly, for example, by means of the generating function technique.

For $\omega$ asymptotically small (the limit we are interested in), the overlapping region $1 \ll m \ll$ $\omega^{-1}$ becomes arbitrarily large. We can then derive matching conditions between the two approximate solutions to fix the coefficients of the Bessel equation and the initial values of the zero-frequency recursion relation. Hence, in the limit of asymptotically small frequency, this procedure gives a complete solution for the ground state of $H$. This solution can be used to compute the expectation value of $(\bar{B}-B)$ in the ground state, which yields the asymptotic form of the density of states $(3.25)$ as $\varepsilon \rightarrow 0$.

We first consider the limit $m \gg 1$. In this limit, we can neglect terms of order one compared to $m$ in Eq. (5.2b) and replace finite differences by derivatives with respect to $m$, i.e., the discrete index $m$ is now treated as a continuous one with $a_{m}^{(1)} \rightarrow a^{(1)}(m)$. This gives

$$
2 \omega a^{(1)}=2\left(m \frac{d^{2} a^{(1)}}{d m^{2}}+\frac{d a^{(1)}}{d m}\right) .
$$

Here, we have assumed that $a^{(1)}$ varies slowly so that $d a^{(1)} / d m$ can be neglected relative to $a^{(1)}$. After the substitution

$$
x^{2}:=4 \omega m,
$$

whereby

$$
\frac{x^{2}}{4 \omega} \gg 1
$$

this differential equation can be transformed into a modified Bessel equation and we obtain the solutions

$$
a^{(1)}(x)=c_{\mathrm{o}, 1}^{(1)} K_{0}(x)+c_{\mathrm{o}, 2}^{(1)} I_{0}(x),
$$

where $I_{0}(x)$ is the modified Bessel function of the first kind and $K_{0}(x)$ the modified Bessel function of the second kind (see chapter 9.6. in [92]). We shall demand that $a_{m}^{(1)}$ decays to zero as $m \rightarrow \infty$, i.e., we must set

$$
c_{\mathrm{o}, 2}^{(1)}=0
$$

in Eq. (5.5). The remaining coefficient $c_{\mathrm{o}, 1}^{(1)}$ of the modified Bessel function $K_{0}$ is determined by matching the solution 5.5 to the one for $m \ll \omega^{-1}$. The coefficient $c_{\mathrm{o}, 1}^{(1)}$ of the modified Bessel function $K_{0}$ will thereby acquire an $\omega$ dependence. In order to carry out this matching procedure, we will need the small $x$ behavior of the continuum solution

$$
a^{(1)}(x) \sim-c_{\mathrm{o}, 1}^{(1)}[\ln (x / 2)+\gamma]=-\frac{c_{\mathrm{o}, 1}^{(1)}}{2}[\ln (m \omega)+2 \gamma],
$$

where $\gamma$ is the Euler's constant.

Second, we consider the limit $m \ll \omega^{-1}$, in which case we can safely drop the terms containing $\omega$ in Eqs. (5.1). Equation 5.2b) can then be written as 


$$
\begin{aligned}
0 & =(2 m+3) \Delta a_{m+1}^{(1)}-(2 m+1) \Delta a_{m}^{(1)} \\
& =\Delta\left((2 m+1) \Delta a_{m}^{(1)}\right)
\end{aligned}
$$

with $m=1,2,3, \cdots$, together with the initial condition

$$
3 a_{2}^{(1)}=4 a_{1}^{(1)}-\sqrt{2} .
$$

Integrating Eq. (5.8a) and determining the integration constant by use of Eq. (5.8b), we find the first order difference equation

$$
(2 m+1)\left(a_{m+1}^{(1)}-a_{m}^{(1)}\right)=a_{1}^{(1)}-\sqrt{2}
$$

with $m=1,2,3, \cdots$, whose solution is given in terms of the Digamma function $\Psi$ (for references on the Digamma function $\Psi$, see chapters 6.3 or 8.36 in Refs. [92] or [93], respectively)

with the limiting form

$$
a_{m}^{(1)}=\sqrt{2}+\frac{1}{2}\left(a_{1}^{(1)}-\sqrt{2}\right)(\Psi(m+1 / 2)-\Psi(1 / 2)),
$$

$$
a_{m}^{(1)} \sim \sqrt{2}+\frac{1}{2}\left(a_{1}^{(1)}-\sqrt{2}\right)(\ln m-\Psi(1 / 2))
$$

for large $m \in \mathbb{N}$.

Having solved the recursion relation in the two limits $1 \ll m$ and $m \ll \omega^{-1}$, we now match solutions 5.7) and 5.11) in the overlapping regime $1 \ll m \ll \omega^{-1}$ to fix the coefficient $c_{\mathrm{o}, 1}^{(1)}$ of the modified Bessel function $K_{0}$ and the initial value $a_{1}^{(1)}$ of the $\omega=0$ recursion relation. Equating Eq. (5.7) with Eq. (5.11) yields the two conditions

$$
\begin{aligned}
c_{\mathrm{o}, 1}^{(1)} & =-a_{1}^{(1)}+\sqrt{2}, \\
-c_{\mathrm{o}, 1}^{(1)} \ln \omega-2 c_{\mathrm{o}, 1}^{(1)} \gamma & =2 \sqrt{2}-\left(a_{1}^{(1)}-\sqrt{2}\right) \Psi(1 / 2),
\end{aligned}
$$

for the two unknowns $c_{\mathrm{o}, 1}^{(1)}$ and $a_{1}^{(1)}$. Solving for $c_{\mathrm{o}, 1}^{(1)}$ and $a_{1}^{(1)}$, we get

$$
a_{1}^{(1)}=\sqrt{2}\left(\frac{2}{\ln \left(C_{1} \omega\right)}+1\right), \quad c_{\mathrm{o}, 1}^{(1)}=\frac{-2 \sqrt{2}}{\ln \left(C_{1} \omega\right)}, \quad C_{1}:=\exp (2 \gamma+\Psi(1 / 2)) .
$$

We have thus derived two limiting solutions to the recursion relations 5.1

$$
a_{m}^{(1)}= \begin{cases}-\frac{2 \sqrt{2}}{\ln \left(C_{1} \omega\right)} K_{0}(2 \sqrt{m \omega}), & 1 \ll m, \\ \sqrt{2}\left(1+\frac{\Psi(m+1 / 2)-\Psi(1 / 2)}{\ln \left(C_{1} \omega\right)}\right), & m \ll \omega^{-1} .\end{cases}
$$

Knowledge of the asymptotics (5.14) is sufficient to determine the leading behavior of the density of states $\nu(\varepsilon)$, Eq. (3.25), for asymptotically small energies $\varepsilon$. The density of states in the long wire limit Eq. (3.24) is given by the expectation value of the operator $(\bar{B}-B)$ in the ground state

$$
\begin{aligned}
\nu(\varepsilon) & =\lim _{\omega \rightarrow-\mathrm{i} \varepsilon} \pi^{-1} \operatorname{Re}_{\mathrm{L}}\left\langle\varphi_{0}|(\bar{B}-B)| \varphi_{0}\right\rangle_{\mathrm{R}} \\
& =\lim _{\omega \rightarrow-i \varepsilon} \pi^{-1} \operatorname{Re}\left(1+\sum_{m=1}^{\infty}\left(a_{m}^{(1)}\right)^{2}\right),
\end{aligned}
$$


where we have used Eq. (4.8) and the identity

$$
(\bar{B}-B)|m\rangle_{\mathrm{R}}^{(1)}=|m\rangle_{\mathrm{L}}^{(1)} .
$$

In view of Eq. (5.14), it is necessary to break the sum over $m$ in Eq. (5.15a) into two parts separated by the integer $m_{0}$, with $1 \ll m_{0} \ll \omega^{-1}$. We choose $m_{0}$ to be some fixed number, independent of $\omega$. With this choice and for sufficiently small $\omega$, the sum on the right-hand side of Eq. (5.15a) is dominated by the part $m>m_{0}$, i.e., by the contribution from the first line in Eq. 5.14). (In the limit $\omega \rightarrow 0$, the sum over $m \leq m_{0}$, i.e., the contribution from the second line in Eq. (5.14), is less divergent than the sum over $m>m_{0}$, and can therefore be neglected.) We then find the estimate

$$
\begin{aligned}
{ }_{\mathrm{L}}\left\langle\varphi_{0}|(\bar{B}-B)| \varphi_{0}\right\rangle_{\mathrm{R}} & \approx \frac{8}{\ln ^{2}\left(C_{1} \omega\right)} \int_{m_{0}}^{\infty} d m K_{0}^{2}(2 \sqrt{m \omega}) \\
& =\frac{4 \omega^{-1}}{\ln ^{2}\left(C_{1} \omega\right)} \int_{x_{0}}^{\infty} d x x K_{0}^{2}(x) .
\end{aligned}
$$

This gives the asymptotic behavior of the density of states

$$
\nu(\varepsilon) \propto \lim _{\omega \rightarrow-\mathrm{i} \varepsilon} \operatorname{Re} \frac{1}{\omega \ln ^{2}\left(C_{1} \omega\right)} \sim \frac{1}{\varepsilon\left|\ln ^{3} \varepsilon\right|},
$$

which has precisely the form of Dyson's singularity.

\subsection{Density of states for two channels $N=2$}

For the case of two channels, $N=2$, the recursion relations 4.13 for the coefficients $a_{m}^{(1)}$ and $a_{m}^{(2)}$ reduce to

$$
\begin{aligned}
& 2 \omega a_{m+1}^{(1)}=(2 m+3)\left(-2 a_{m+1}^{(1)}+a_{m+2}^{(1)}+a_{m}^{(1)}\right), \\
& 2 \omega a_{m+1}^{(2)}=(2 m+1)\left(-2 a_{m+1}^{(2)}+a_{m+2}^{(2)}+a_{m}^{(2)}\right),
\end{aligned}
$$

with $m=1,2,3, \cdots$ and with the initial conditions

$$
\begin{aligned}
& 2 \omega a_{1}^{(1)}=+\sqrt{2} 3-6 a_{1}^{(1)}+3 a_{2}^{(1)}, \\
& 2 \omega a_{1}^{(2)}=-\sqrt{2}-2 a_{1}^{(2)}+a_{2}^{(2)} .
\end{aligned}
$$

Alternatively, we can rewrite the two decoupled recursion relations (5.18a) in terms of the finite differences

$$
\Delta a_{m}^{(i)}:=a_{m+1}^{(i)}-a_{m}^{(i)}, \quad \Delta^{2} a_{m}^{(i)}:=a_{m+2}^{(i)}-2 a_{m+1}^{(i)}+a_{m}^{(i)}, \quad i=1,2 .
$$

With this, Eq. (5.18a) becomes

$$
\begin{aligned}
& 2 \omega a_{m}^{(1)}=(2 m+3) \Delta^{2} a_{m}^{(1)}-2 \omega \Delta a_{m}^{(1)}, \\
& 2 \omega a_{m}^{(2)}=(2 m+1) \Delta^{2} a_{m}^{(2)}-2 \omega \Delta a_{m}^{(2)} .
\end{aligned}
$$

To analyze these equations, we follow the same strategy as in the previous section for the $N=1$ case and solve the Eqs. (5.18) first in the $m \gg 1$ limit and then in the $m \ll \omega^{-1}$ limit. 
We begin with the $m \gg 1$ limit. Treating $m$ as a continuous index, we replace finite differences by derivatives in Eq. 5.18d), i.e., we let $a_{m}^{(i)} \rightarrow a^{(i)}(m)$ with $i=1,2$. This gives

$$
2 \omega a^{(i)}=2 m \frac{d^{2} a^{(i)}}{d m^{2}} . \quad i=1,2 .
$$

Here, $a^{(i)}$ is assumed to be slowly varying so that we can neglect $d a^{(i)} / d m$ compared to $a^{(i)}$. After the substitution

$$
x^{2}:=4 \omega m,
$$

whereby

$$
\frac{x^{2}}{4 \omega} \gg 1,
$$

Eq. (5.19) reduces to a Bessel-type equation whose solution is given in terms of the modified Bessel functions $K_{1}(x)$ and $I_{1}(x)$,

$$
a^{(i)}(x)=c_{\mathrm{e}, 1}^{(i)} \frac{x}{2} K_{1}(x)+c_{\mathrm{e}, 2}^{(i)} \frac{x}{2} I_{1}(x), \quad i=1,2,
$$

with the coefficients $c_{\mathrm{e}, j}^{(i)}$ with $i=1,2$ of the modified Bessel functions $K_{1}$ when $j=1$ and $I_{1}$ when $j=2$. We shall demand that $a_{m}^{(i)}$ with $i=1,2$ decay to zero as $m \rightarrow \infty$, i.e., we must set

$$
c_{\mathrm{e}, 2}^{(i)}=0 .
$$

in Eq. 5.21). The remaining coefficients $c_{\mathrm{e}, 1}^{(i)}$ with $i=1,2$ of the modified Bessel function $K_{1}$ will be fixed by matching solutions (5.21) to the small $m \omega$ solution. In order to do so we will need the small $x$ behavior of Eq. (5.21)

$$
\begin{aligned}
a^{(i)}(x) & \sim \frac{1}{2} c_{\mathrm{e}, 1}^{(i)}+c_{\mathrm{e}, 1}^{(i)} \frac{x^{2}}{8}\left[\ln \left(x^{2} / 4\right)+2 \gamma-1\right] \\
& =\frac{1}{2} c_{\mathrm{e}, 1}^{(i)}+c_{\mathrm{e}, 1}^{(i)} \frac{m \omega}{2} \ln \left(C_{0}^{(\mathrm{e})} m \omega\right),
\end{aligned}
$$

with $i=1,2$ and where

$$
C_{0}^{(\mathrm{e})}:=\exp (2 \gamma-1)
$$

Next, we turn to the limit $m \omega \ll 1$, in which we can neglect the $\omega$ term in Eq. (5.18d). If so, Eq. 5.18d becomes

$$
\begin{aligned}
& 0=(2 m+3) \Delta^{2} a_{m}^{(1)}, \\
& 0=(2 m+1) \Delta^{2} a_{m}^{(2)},
\end{aligned}
$$

together with the initial conditions

$$
\begin{aligned}
& 2 a_{1}^{(1)}=+\sqrt{2}+a_{2}^{(1)}, \\
& 2 a_{1}^{(2)}=-\sqrt{2}+a_{2}^{(2)} .
\end{aligned}
$$

The solution of Eqs. (5.24a) is given by

$$
\begin{aligned}
& a_{m}^{(1)}=m a_{1}^{(1)}-(m-1) \sqrt{2}, \\
& a_{m}^{(2)}=m a_{1}^{(2)}+(m-1) \sqrt{2},
\end{aligned}
$$

where we have fixed two initial values by use of Eq. (5.24b).

Having solved the two decoupled recursion relations (5.18) in the two limits $m \gg 1$ and $m \ll \omega^{-1}$, we now match solutions (5.21) and (5.25) in the overlapping region in order to fix the 
coefficients $c_{\mathrm{e}, 1}^{(i)}$ of the modified Bessel functions $K_{1}$ and initial values $a_{1}^{(i)}$ with $i=1,2$ of the $\omega=0$ recursion relation. Equating (5.23) with (5.25) and matching equal powers of $m$ (whereby we neglect $\ln m$ compared to $\ln \omega$ ) gives the two equations

$$
\begin{aligned}
+\sqrt{2} & =\frac{1}{2} c_{\mathrm{e}, 1}^{(1)}, \\
a_{1}^{(1)}-\sqrt{2} & =c_{\mathrm{e}, 1}^{(1)} \frac{\omega}{2} \ln \left(C_{0}^{(\mathrm{e})} \omega\right),
\end{aligned}
$$

for the two unknowns $c_{\mathrm{e}, 1}^{(1)}$ and $a_{1}^{(1)}$ and the two equations

$$
\begin{aligned}
-\sqrt{2} & =\frac{1}{2} c_{\mathrm{e}, 1}^{(2)}, \\
a_{1}^{(2)}+\sqrt{2} & =c_{\mathrm{e}, 1}^{(2)} \frac{\omega}{2} \ln \left(C_{0}^{(\mathrm{e})} \omega\right),
\end{aligned}
$$

for the two unknowns $c_{\mathrm{e}, 1}^{(2)}$ and $a_{1}^{(2)}$. Solving for $a_{1}^{(1)}$ and $a_{1}^{(2)}$, respectively, we obtain

$$
\begin{aligned}
& a_{1}^{(1)}=+\sqrt{2}\left[1+\omega \ln \left(C_{0}^{(\mathrm{e})} \omega\right)\right], \\
& a_{1}^{(2)}=-\sqrt{2}\left[1+\omega \ln \left(C_{0}^{(\mathrm{e})} \omega\right)\right] .
\end{aligned}
$$

We have thus derived two limiting solutions to the recursion relations (5.18)

$$
\begin{aligned}
& a_{m}^{(1)}= \begin{cases}+2 \sqrt{2 m \omega} K_{1}(2 \sqrt{m \omega}), & 1 \ll m, \\
+m \sqrt{2}\left[\omega \ln \left(C_{0}^{(\mathrm{e})} \omega\right)\right]+\sqrt{2}, & m \ll \omega^{-1},\end{cases} \\
& a_{m}^{(2)}= \begin{cases}-2 \sqrt{2 m \omega} K_{1}(2 \sqrt{m \omega}), & 1 \ll m, \\
-m \sqrt{2}\left[\omega \ln \left(C_{0}^{(\mathrm{e})} \omega\right)\right]-\sqrt{2}, & m \ll \omega^{-1} .\end{cases}
\end{aligned}
$$

In the long wire limit, the density of states is given by the expectation value of the operator $(\bar{B}-B)$ in the ground state [see Eq. (3.24)]

$$
\begin{aligned}
\nu(\varepsilon) & =\lim _{\omega \rightarrow-i \varepsilon} \pi^{-1} \operatorname{Re}_{\mathrm{L}}\left\langle\varphi_{0}|(\bar{B}-B)| \varphi_{0}\right\rangle_{\mathrm{R}} \\
& =\lim _{\omega \rightarrow-i \varepsilon} \pi^{-1} \operatorname{Re}\left[2+\sum_{m=1}^{\infty}\left(\frac{\left(a_{m}^{(1)}\right)^{2}}{2 m+1}-\frac{\left(a_{m}^{(2)}\right)^{2}}{2 m-1}\right)\right] .
\end{aligned}
$$

Here, we have used Eq. (4.8), the identity $(\bar{B}-B)|0\rangle=2|0\rangle$ and

$$
\begin{aligned}
& (\bar{B}-B)|m\rangle_{\mathrm{R}}^{(1)}=+|m\rangle_{\mathrm{R}}^{(1)}+|m\rangle_{\mathrm{L}}^{(1)}, \\
& (\bar{B}-B)|m\rangle_{\mathrm{R}}^{(2)}=-|m\rangle_{\mathrm{R}}^{(2)}-|m\rangle_{\mathrm{L}}^{(2)} .
\end{aligned}
$$

We want to estimate the leading behavior of the density of states for two scattering channels, $N=2$, and for asymptotically small energies $\varepsilon$, i.e. $\omega \ll 1$. Thereto, we break the sum over $m$ in Eq. (5.29) into two parts separated by the integer $1 \ll m_{0} \sim \omega^{-1}$. With the choice $m_{0}=\omega^{-1}$, we find that the sum in Eq. (5.29) is dominated by the small $m \ll 1 / \omega$ solutions in Eq. (5.28) [the rest of the sum is easily shown to be $\mathcal{O}(\omega)$ ], 


$$
{ }_{\mathrm{L}}\left\langle\varphi_{0}|(\bar{B}-B)| \varphi_{0}\right\rangle_{\mathrm{R}} \approx 2+2 \sum_{m=1}^{1 / \omega}\left(\frac{1}{2 m+1}-\frac{1}{2 m-1}\right)\left[1+m \omega \ln \left(C_{0}^{(\mathrm{e})} \omega\right)\right]^{2} .
$$

This sum over $m$ can be computed exactly by means of a telescopic expansion. We find

$$
\begin{aligned}
{ }_{\mathrm{L}}\left\langle\varphi_{0}|(\bar{B}-B)| \varphi_{0}\right\rangle_{\mathrm{R}} \approx & 2-\frac{4}{2+\omega}-2 \omega\left(\frac{1+\omega}{2+\omega}\right) \ln ^{2}\left(C_{0}^{(\mathrm{e})} \omega\right)+\omega[2-2 \gamma \\
& \left.-2 \ln 4-\Psi\left(\omega^{-1}+1 / 2\right)-\Psi\left(\omega^{-1}+3 / 2\right)\right] \ln \left(C_{0}^{(\mathrm{e})} \omega\right)
\end{aligned}
$$

To compute the leading behavior of the density of states it is sufficient to retain only the lowest order in $\omega$. This gives

$$
{ }_{\mathrm{L}}\left\langle\varphi_{0}|(\bar{B}-B)| \varphi_{0}\right\rangle_{\mathrm{R}} \propto-\omega \ln ^{2} \omega+\text { higher order terms. }
$$

We thus find the estimate

$$
\nu(\varepsilon) \propto \lim _{\omega \rightarrow-\mathrm{i} \varepsilon} \pi^{-1} \operatorname{Re}\left(-\omega \ln ^{2} \omega\right) \propto \varepsilon|\ln \varepsilon|
$$

for the density of states in the thermodynamic limit.

\section{Evaluation of the density of states when $N=1,2,3, \cdots$}

We are going to compute the density of states $\nu(\varepsilon)$ from Eq. (3.25) to leading order in the positive dimensionless energy $\varepsilon \ll 1$ by solving the recursion relation $(4.13)$ for an arbitrary number $N$ of channels. We begin with the case of $N$ odd and proceed with the case of $N$ even. In doing so, we are going to reproduce the parity effect 1.1 in the density of states that was obtained for the first time using the DMPK approach in Refs. [82] and [83].

\subsection{Density of states for an odd number $N$ of channels}

The calculation of the density of states for an odd number $N$ of channels follows along the lines of the $N=1$ case with the caveat that for $N>1$ the recursion relations 4.13 no longer decouple. This difference complicates the calculation considerably. To overcome this difficulty we introduce a linear transformation of the coefficients $a_{m}^{(n)}$ that approximately decouples the recursion relations (4.13) in the two regimes $m \gg N$ and $m \ll \omega^{-1}$. That is, we introduce

$$
\begin{array}{ll}
b_{m}^{(2 k+1)}=\sum_{n=0}^{(N-1) / 2}\left[\mathcal{M}_{\mathrm{o} ; N, 1}\right]_{n}^{k} a_{m}^{(2 n+1)}, & k=0,1, \cdots, \frac{N-1}{2}, \\
b_{m}^{(2 k+2)}=\sum_{n=0}^{(N-3) / 2}\left[\mathcal{M}_{\mathrm{o} ; N, 2}\right]_{n}^{k} a_{m}^{(2 n+2)}, & k=0,1, \cdots, \frac{N-3}{2},
\end{array}
$$

with $m=1,2,3, \cdots$ and the transformation matrices 


$$
\begin{aligned}
& {\left[\mathcal{M}_{\mathrm{o} ; N, 1}\right]_{n}^{k}:=(-1)^{n} \frac{(2 n) !(N-2 n-1) !}{n !\left(\frac{N-1}{2}-n\right) !}{ }_{3} F_{2}\left[\begin{array}{c}
-k, k,-n \\
\frac{1}{2}, \frac{1-N}{2} ; 1
\end{array}\right],} \\
& {\left[\mathcal{M}_{\mathrm{o} ; N, 2}\right]_{n}^{k}:=(-1)^{n} \frac{(2 n+1) !(N-2 n-2) !}{n !\left(\frac{N-3}{2}-n\right) !}{ }_{3} F_{2}\left[\begin{array}{c}
-k, k+2,-n \\
\frac{3}{2}, \frac{3-N}{2}
\end{array}\right] 1 .}
\end{aligned}
$$

Here, the symbol ${ }_{3} F_{2}$ denotes a generalized hypergeometric function (see for example Ref. [93]). It is also possible to express the $a_{m}^{(n)}$, s in terms of the $b_{m}^{(k)}$, s,

$$
\begin{array}{ll}
a_{m}^{(2 n+1)}=\sum_{k=0}^{(N-1) / 2}\left[\mathcal{M}_{\mathrm{o} ; N, 1}^{-1}\right]_{k}^{n} b_{m}^{(2 k+1)}, & n=0,1, \cdots, \frac{N-1}{2}, \\
a_{m}^{(2 n+2)}=\sum_{k=0}^{(N-3) / 2}\left[\mathcal{M}_{\mathrm{o} ; N, 2}^{-1}\right]_{k}^{n} b_{m}^{(2 k+2)}, & n=0,1, \cdots, \frac{N-3}{2} .
\end{array}
$$

With these definitions, we are going to rewrite the recursion relations $(4.13)$ in a form that decouples both in the limit $\omega=0$ and in the large $m$ limit.

To see this, we first make use of identities (B.3a and (B.3b) to combine Eqs. (4.13) and (6.1) into the recursion relations

$$
\begin{aligned}
4 m \omega b_{m}^{(2 k+1)}= & {\left[-4 m(2 m-1+N)+\left(N-1-4 k^{2}\right)\right] b_{m}^{(2 k+1)} } \\
& +2 m(2 m+1) b_{m+1}^{(2 k+1)}+(2 m-2+N)(2 m+N-1) b_{m-1}^{(2 k+1)} \\
& -4 \omega \sum_{n=0}^{(N-1) / 2} n\left[\mathcal{M}_{\mathrm{o} ; N, 1}\right]_{n}^{k} a_{m}^{(2 n+1)}
\end{aligned}
$$

with $k=0,1, \cdots,(N-1) / 2$ and

$$
\begin{aligned}
4 m \omega b_{m}^{(2 k+2)}=\{ & \left.-4 m(2 m-3+N)+\left[3 N-5-4(k+1)^{2}\right]\right\} b_{m}^{(2 k+2)} \\
& +2 m(2 m-1) b_{m+1}^{(2 k+2)}+(2 m-3+N)(2 m-2+N) b_{m-1}^{(2 k+2)} \\
& -4 \omega \sum_{n=0}^{(N-3) / 2} n\left[\mathcal{M}_{\mathrm{o} ; N, 2}\right]_{n}^{k} a_{m}^{(2 n+2)}
\end{aligned}
$$

with $k=0,1, \cdots,(N-3) / 2$ and $m=2,3, \cdots$. Here, the initial conditions are

$$
\begin{aligned}
4 \omega b_{1}^{(2 k+1)}= & +\sqrt{2} N(N+1)-\left(3 N+5+4 k^{2}\right) b_{1}^{(2 k+1)}+6 b_{2}^{(2 k+1)} \\
& -4 \omega \sum_{n=0}^{(N-1) / 2} n\left[\mathcal{M}_{\mathrm{o} ; N, 1}\right]_{n}^{k} a_{1}^{(2 n+1)}
\end{aligned}
$$

with $k=0,1, \cdots,(N-1) / 2$ and

$$
\begin{aligned}
4 \omega b_{1}^{(2 k+2)}= & -\sqrt{2} N(N-1)-\left[N+1+4(k+1)^{2}\right] b_{1}^{(2 k+2)}+2 b_{2}^{(2 k+2)} \\
& -4 \omega \sum_{n=0}^{(N-3) / 2} n\left[\mathcal{M}_{\mathrm{o} ; N, 2}\right]_{n}^{k} a_{1}^{(2 n+2)}
\end{aligned}
$$

with $k=0,1, \cdots,(N-3) / 2$. We aim at a solution of Eq. 6.2) for asymptotically small frequency $\omega$. As in Sec. 5.1, our strategy is to solve Eq. (6.2) in the two limits $N \ll m$ and $m \ll$ 
$\omega^{-1}$. In the former limit, $m \gg N$, we treat $m$ as a continuous variable. Then, Eq. (6.2) decouples, provided we assume that $a_{m}^{(n)}$ decays rapidly for $m \gg N$. In the other limit, $m \ll \omega^{-1}$, we can neglect $\omega$ in Eq. (6.2), which again decouples. In this way, it is possible to find approximate solutions in the two regions $N \ll m$ and $m \ll \omega^{-1}$ that are uniquely fixed up to some multiplicative coefficients and initial values, respectively. For asymptotically small $\omega$, the overlap between these two regions $N \ll m \ll \omega^{-1}$ is large. We can then match the two approximate solutions in the overlapping region. This gives a unique and approximate solution for the ground state wave function, which in turn determines the density of states (3.25) in the long wire limit.

\subsubsection{Solution when $m \gg N$}

First, we treat the limit $m \gg N$. If we assume that $a_{m}^{(n)}$ decays rapidly for large $m$, we can drop the last line on the right-hand side of Eqs. (6.2a) and (6.2b), respectively. Consequently, in terms of the finite differences

$$
\Delta b_{m}^{(i)}:=b_{m+1}^{(i)}-b_{m}^{(i)}, \quad \quad \Delta^{2} b_{m}^{(i)}:=b_{m+2}^{(i)}-2 b_{m+1}^{(i)}+b_{m}^{(i)},
$$

with $i=1, \cdots, N$, the recursion relations Eqs. (6.2a) and and (6.2b) read

$$
\begin{aligned}
(m+1) 4 \omega\left(\Delta b_{m}^{(2 k+1)}+b_{m}^{(2 k+1)}\right)= & -2(2 m+2)(N-2) \Delta b_{m}^{(2 k+1)} \\
& +(2 m+2)(2 m+3) \Delta^{2} b_{m}^{(2 k+1)} \\
& +(N-1)(N-2) b_{m}^{(2 k+1)} \\
& +\left(N-1-4 k^{2}\right)\left(\Delta b_{m}^{(2 k+1)}+b_{m}^{(2 k+1)}\right)
\end{aligned}
$$

with $k=0,1, \cdots,(N-1) / 2$ and

$$
\begin{aligned}
(m+1) 4 \omega\left(\Delta b_{m}^{(2 k+2)}+b_{m}^{(2 k+2)}\right)= & -2(2 m+2)(N-2) \Delta b_{m}^{(2 k+2)} \\
& +(2 m+2)(2 m+1) \Delta^{2} b_{m}^{(2 k+2)} \\
& +(N-2)(N-3) b_{m}^{(2 k+2)} \\
& +\left[3 N-5-4(k+1)^{2}\right]\left(\Delta b_{m}^{(2 k+2)}+b_{m}^{(2 k+2)}\right)
\end{aligned}
$$

with $k=0,1, \cdots,(N-3) / 2$. In the limit $m \gg N$, we can neglect terms of order $N$ compared to $m$ and replace finite differences by derivatives. In place of Eq. (6.4) and if we assume that $b_{m}^{(i)} \rightarrow b^{(i)}(m)$ with $i=1,2, \cdots, N$ is slowly varying, we get

$$
4 m \omega b^{(2 k+1)}=-4 m(N-2) \frac{d b^{(2 k+1)}}{d m}+4 m^{2} \frac{d^{2} b^{(2 k+1)}}{d m^{2}}+\left[(N-1)^{2}-4 k^{2}\right] b^{(2 k+1)}
$$

with $k=0,1, \cdots,(N-1) / 2$ and

$$
4 m \omega b^{(2 k+2)}=-4 m(N-2) \frac{d b^{(2 k+2)}}{d m}+4 m^{2} \frac{d^{2} b^{(2 k+2)}}{d m^{2}}+\left[(N-1)^{2}-4(k+1)^{2}\right] b^{(2 k+2)}
$$

with $k=0,1, \cdots,(N-3) / 2$. By use of the substitution

$$
x^{2}:=4 \omega m,
$$

whereby

$$
\frac{x^{2}}{4 \omega} \gg N
$$


we find that the solutions to Eq. 6.5) are given by the linear combinations of

$$
b^{(2 k+1)}(x)=c_{\mathrm{o}, 1}^{(2 k+1)}\left(\frac{x}{2}\right)^{N-1} K_{2 k}(x)+c_{\mathrm{o}, 2}^{(2 k+1)}\left(\frac{x}{2}\right)^{N-1} I_{2 k}(x)
$$

with $k=0,1, \cdots,(N-1) / 2$ and

$$
b^{(2 k+2)}(x)=c_{\mathrm{o}, 1}^{(2 k+2)}\left(\frac{x}{2}\right)^{N-1} K_{2 k+2}(x)+c_{\mathrm{o}, 2}^{(2 k+2)}\left(\frac{x}{2}\right)^{N-1} I_{2 k+2}(x)
$$

with $k=0,1, \cdots,(N-3) / 2$, of modified Bessel functions $K_{2 k}$ and $I_{2 k}$. We shall demand that $b_{m}^{(i)}$ with $i=1, \cdots, N$ decay to zero as $m \rightarrow \infty$, i.e., we must set

$$
c_{\mathrm{o}, 2}^{(i)}=0
$$

with $i=1, \cdots, N$ in Eq. 6.7). The remaining $N$ coefficients $c_{\mathrm{o}, 1}^{(i)}$ with $i=1, \cdots, N$ of the modified Bessel functions $K_{0}, K_{2}, K_{4}, \cdots, K_{N-1}$ are fixed by matching solutions 6.7) to the solutions in the $m \ll \omega^{-1}$ region. Thereto, we need to extract the terms that are of order $x^{N+2 k-1}$ and $x^{N+2 k-3}$ from the expansion of Eq. (6.7a) and the terms that are of order $x^{N+2 k+1}$ and $x^{N+2 k-1}$ from the expansion of Eq. (6.7b), when $x \ll 1$. This gives

$$
\begin{gathered}
b^{(1)}(x) \sim c_{\mathrm{o}, 1}^{(1)}\left(\frac{x}{2}\right)^{N-1} \frac{1}{2}\left(-2 \gamma-\ln \frac{x^{2}}{4}\right) \\
=-c_{\mathrm{o}, 1}^{(1)}(m \omega)^{\frac{N-1}{2}} \frac{1}{2} \ln \left(C_{0}^{(\mathrm{o})} m \omega\right), \\
b^{(2 k+1)}(x) \sim c_{\mathrm{o}, 1}^{(2 k+1)}\left[\frac{(x / 2)^{N+2 k-1}}{2(2 k) !}\left(-\gamma-\ln \frac{x^{2}}{4}+\Psi(2 k+1)\right)-\frac{(x / 2)^{N+2 k-3}}{2(2 k-1) !}\right] \\
=c_{\mathrm{o}, 1}^{(2 k+1)}\left[-\frac{(m \omega)^{\frac{N-1}{2}+k}}{2(2 k) !} \ln \left(C_{k}^{(\mathrm{o})} m \omega\right)-\frac{(m \omega)^{\frac{N-1}{2}+k-1}}{2(2 k-1) !}\right],
\end{gathered}
$$

with $k=1,2, \cdots,(N-1) / 2$, and

$$
\begin{aligned}
b^{(2 k+2)}(x) & \sim c_{\mathrm{o}, 1}^{(2 k+2)}\left[\frac{(x / 2)^{N+2 k+1}}{2(2 k+2) !}\left(-\gamma-\ln \frac{x^{2}}{4}+\Psi(2 k+3)\right)-\frac{(x / 2)^{N+2 k-1}}{2(2 k+1) !}\right] \\
& =c_{\mathrm{o}, 1}^{(2 k+2)}\left[-\frac{(m \omega)^{\frac{N+1}{2}+k}}{2(2 k+2) !} \ln \left(C_{k+1}^{(\mathrm{o})} m \omega\right)-\frac{(m \omega)^{\frac{N+1}{2}+k-1}}{2(2 k+1) !}\right],
\end{aligned}
$$

with $k=0,1, \cdots,(N-3) / 2$, and where

$$
C_{k}^{(\text {o) }}:=\exp (\gamma-\Psi(2 k+1)) .
$$

(For references on the Digamma function $\Psi$, see chapters 6.3 or 8.36 in Refs. [92] or [93], respectively.)

\subsubsection{Solution when $1 \leq m \ll \omega^{-1}$}

Second, we treat the limit $1 \leq m \ll \omega^{-1}$, in which case we can neglect the $\omega$ terms in Eq. 6.2. In doing so, Eq. 6.2) becomes

$$
\begin{aligned}
0= & {\left[-4 m(2 m-1+N)+\left(N-1-4 k^{2}\right)\right] b_{m}^{(2 k+1)}+2 m(2 m+1) b_{m+1}^{(2 k+1)} } \\
& +(2 m-2+N)(2 m-1+N) b_{m-1}^{(2 k+1)}
\end{aligned}
$$


with $k=0,1, \cdots,(N-1) / 2$ and

$$
\begin{aligned}
0=\{ & \left.-4 m(2 m-3+N)+\left[3 N-5-4(k+1)^{2}\right]\right\} b_{m}^{(2 k+2)}+2 m(2 m-1) b_{m+1}^{(2 k+2)} \\
& +(2 m-3+N)(2 m-2+N) b_{m-1}^{(2 k+2)}
\end{aligned}
$$

with $k=0,1, \cdots,(N-3) / 2, m=2,3, \cdots$, together with the initial conditions

$$
0=+\sqrt{2} N(N+1)-\left(5+3 N+4 k^{2}\right) b_{1}^{(2 k+1)}+6 b_{2}^{(2 k+1)}
$$

with $k=0,1, \cdots,(N-1) / 2$ and

$$
0=-\sqrt{2} N(N-1)-\left[N+1+4(k+1)^{2}\right] b_{1}^{(2 k+2)}+2 b_{2}^{(2 k+2)}
$$

with $k=0,1, \cdots,(N-3) / 2$. The solution to the recursion relation (6.10) can be expressed in terms of generalized hypergeometric functions

$$
\begin{aligned}
b_{m}^{(1)} & =\mathcal{C}_{0}^{(\mathrm{o}, 1)} \sum_{l=0}^{m-1} \frac{\left(m-1-l+\frac{N-1}{2}\right) !}{(2 l+1)(m-1-l) !}+\mathcal{C}_{0}^{(\mathrm{o}, 2)} \frac{\left(m-1+\frac{N}{2}\right) !}{\left(m-\frac{1}{2}\right) !}, \\
b_{m}^{(2 k+1)}= & \mathcal{C}_{k}^{(\mathrm{o}, 1)} \frac{\left(m+k-\frac{3}{2}+\frac{N}{2}\right) !}{(m-1) !}{ }_{3} F_{2}\left[\begin{array}{l}
\frac{1}{2}-k, 1-k, 1-m \\
\frac{3}{2}, \frac{3}{2}-k-m-\frac{N}{2} ; 1
\end{array}\right] \\
& +\mathcal{C}_{k}^{(\mathrm{o}, 2)} \frac{\left(m+k-1+\frac{N}{2}\right) !}{\left(m-\frac{1}{2}\right) !}{ }_{3} F_{2}\left[\begin{array}{l}
\frac{1}{2}-k,-k, \frac{1}{2}-m \\
\frac{1}{2}, 1-k-m-\frac{N}{2} ; 1
\end{array}\right]
\end{aligned}
$$

with $k=1,2, \cdots,(N-1) / 2$, and

$$
\begin{aligned}
b_{m}^{(2 k+2)}= & \left.\mathrm{e}_{k}^{(\mathrm{o}, 3)} \frac{\left(m+k-\frac{1}{2}+\frac{N}{2}\right) !}{(m-1) !}{ }_{3} F_{2}\left[\begin{array}{c}
-\frac{1}{2}-k,-1-k, 1-m \\
\frac{1}{2}, \frac{1}{2}-k-m-\frac{N}{2}
\end{array}\right] 1\right] \\
& +\mathrm{C}_{k}^{(\mathrm{o}, 4)} \frac{\left(m+k-1+\frac{N}{2}\right) !}{\left(m-\frac{3}{2}\right) !}{ }_{3} F_{2}\left[\begin{array}{c}
-\frac{1}{2}-k,-k, \frac{3}{2}-m \\
\frac{3}{2}, 1-k-m-\frac{N}{2}
\end{array} ; 1\right]
\end{aligned}
$$

with $k=0,1, \cdots,(N-3) / 2$ and $m=1,2,3, \cdots$. The prefactors $\mathcal{C}_{k}^{(\mathrm{o}, i)} i=1,2,3,4$ to the hypergeometric function ${ }_{3} F_{2}$ are determined by the initial conditions (6.10d) and (6.10d),

$$
\begin{gathered}
\mathcal{C}_{0}^{(\mathrm{o}, 1)}=\frac{b_{1}^{(1)}-\sqrt{2} N}{\left(\frac{N-1}{2}\right) !}, \quad \mathcal{C}_{0}^{(\mathrm{o}, 2)}=\frac{\sqrt{2 \pi}}{\left(\frac{N-2}{2}\right) !} \\
\left.\mathcal{C}_{k}^{(\mathrm{o}, 1)}=\frac{1}{\left(k+\frac{N-1}{2}\right) !}\left\{b_{1}^{(2 k+1)}-\mathcal{C}_{k}^{(\mathrm{o}, 2)} \frac{2}{\sqrt{\pi}}\left(k+\frac{N}{2}\right) !_{3} F_{2}\left[\begin{array}{c}
\frac{1}{2}-k,-k,-\frac{1}{2} \\
\frac{1}{2},-k-\frac{N}{2}
\end{array}\right] 1\right]\right\} \\
\mathcal{C}_{k}^{(\mathrm{o}, 2)}=\sqrt{\pi} 2^{k+\frac{1}{2}} \frac{(N-2 k-1) ! !}{(N-1) ! !\left(\frac{N-2}{2}\right) !}
\end{gathered}
$$


with $k=1,2, \cdots,(N-1) / 2$, and

$$
\begin{aligned}
\mathcal{C}_{k}^{(\mathrm{o}, 3)} & =\frac{1}{\left(k+\frac{N+1}{2}\right) !}\left\{b_{1}^{(2 k+2)}-\mathcal{C}_{k}^{(\mathrm{o}, 4)} \frac{1}{\sqrt{\pi}}\left(k+\frac{N}{2}\right) !_{3} F_{2}\left[\begin{array}{c}
-\frac{1}{2}-k,-k, \frac{1}{2} \\
\frac{3}{2},-k-\frac{N}{2} ; 1
\end{array}\right]\right\}, \\
\mathcal{C}_{k}^{(\mathrm{o}, 4)} & =\sqrt{\pi} 2^{k+\frac{3}{2}} \frac{(N-2 k-3) ! !}{(N-3) ! !\left(\frac{N-2}{2}\right) !}
\end{aligned}
$$

with $k=0,1, \cdots,(N-3) / 2$.

In order to match this solution when $1 \leq m \ll 1 / \omega$ to the solution when $N \ll m$, we need the leading and subleading terms of the large $m$ behavior of $b_{m}^{(2 k+1)}$ and $b_{m}^{(2 k+2)}$. They are

$$
b_{m}^{(1)} \simeq \frac{\mathcal{C}_{0}^{(\mathrm{o}, 1)}}{2} m^{\frac{N-1}{2}} \ln m+\bigodot_{0}^{(\mathrm{o})} m^{\frac{N-1}{2}},
$$

with $\mathrm{C}_{0}^{(\mathrm{o})}$ some number that depends on $N$,

$$
\begin{aligned}
b_{m}^{(2 k+1)} \simeq & 4^{k-\frac{1}{2}}\left(\frac{\mathcal{C}_{k}^{(\mathrm{o}, 1)}}{2 k}+\bigodot_{k}^{(\mathrm{o}, 2)}\right) m^{\frac{N-1}{2}+k} \\
& +4^{k-2}(N+2 k-1)(N-1)\left(\frac{\mathcal{C}_{k}^{(\mathrm{o}, 1)}}{2 k}+\bigodot_{k}^{(\mathrm{o}, 2)}\right) m^{\frac{N-1}{2}+k-1}
\end{aligned}
$$

with $k=1,2, \cdots,(N-1) / 2$, and

$$
\begin{aligned}
b_{m}^{(2 k+2)} \simeq & 4^{k+\frac{1}{2}}\left(\mathcal{C}_{k}^{(\mathrm{o}, 3)}+\frac{\mathcal{C}_{k}^{(\mathrm{o}, 4)}}{2 k+2}\right) m^{\frac{N+1}{2}+k} \\
& +4^{k-1}(N+2 k+1)(N-3)\left(\mathrm{C}_{k}^{(\mathrm{o}, 3)}+\frac{\mathcal{C}_{k}^{(\mathrm{o}, 4)}}{2 k+2}\right) m^{\frac{N+1}{2}+k-1}
\end{aligned}
$$

with $k=0,1, \cdots,(N-3) / 2$, to leading and subleading order in $m$.

\subsubsection{Matching solutions when $N \ll m \ll \omega^{-1}$}

Having solved the recursion relations (6.2) in the two limits $N \ll m$ and $1 \ll m \ll \omega^{-1}$, we are going to match the $m=x^{2} /(4 \omega)$ dependences of the solutions (6.9) and (6.13) in the overlapping region $N \ll m \ll \omega^{-1}$, where both solutions are valid. We start with the coefficient $b_{m}^{(1)}$. Matching Eq. (6.13a) with Eq. 6.9a) gives the two equations

$$
\begin{aligned}
\mathcal{C}_{0}^{(\mathrm{o})} & =-\frac{c_{\mathrm{o}, 1}^{(1)}}{2} \omega^{\frac{N-1}{2}} \ln \left(C_{0}^{(\mathrm{o})} \omega\right), \\
\mathcal{C}_{0}^{(\mathrm{o}, 1)} & =-c_{\mathrm{o}, 1}^{(1)} \omega^{\frac{N-1}{2}}
\end{aligned}
$$

for the two unknowns $b_{1}^{(1)}$ and $c_{\mathrm{o}, 1}^{(1)}$ with the solutions 


$$
\begin{aligned}
& c_{\mathrm{o}, 1}^{(1)}=\frac{-2 \mathfrak{C}_{0}^{(\mathrm{o})}}{\omega^{\frac{N-1}{2}} \ln \left(C_{0}^{(\mathrm{o})} \omega\right)}, \\
& b_{1}^{(1)}=\frac{2 \mathcal{C}_{0}^{(\mathrm{o})}\left(\frac{N-1}{2}\right) !}{\ln \left(C_{0}^{(\mathrm{o})} \omega\right)}+\sqrt{2} N .
\end{aligned}
$$

Matching Eq. (6.13b) with Eq. (6.9b) gives two equations (whereby we neglect $\ln m$ and $\ln C_{k}^{(\mathrm{o})}$ compared to $\ln \omega$ )

$$
\begin{aligned}
-c_{\mathrm{o}, 1}^{(2 k+1)} \frac{\omega^{\frac{N-1}{2}+k}}{2(2 k) !} \ln \omega & =4^{k-\frac{1}{2}}\left(\frac{\mathfrak{C}_{k}^{(\mathrm{o}, 1)}}{2 k}+\mathcal{C}_{k}^{(\mathrm{o}, 2)}\right), \\
-c_{\mathrm{o}, 1}^{(2 k+1)} \frac{\omega^{\frac{N-1}{2}}+k-1}{2(2 k-1) !} & =4^{k-2}(N+2 k-1)(N-1)\left(\frac{\mathfrak{C}_{k}^{(\mathrm{o}, 1)}}{2 k}+\mathcal{C}_{k}^{(\mathrm{o}, 2)}\right),
\end{aligned}
$$

for the two unknowns $b_{1}^{(2 k+1)}$ and $c_{\mathrm{o}, 1}^{(2 k+1)}$, while matching Eq. (6.13g) with Eq. (6.9d) gives two equations

$$
\begin{aligned}
-c_{\mathrm{o}, 1}^{(2 k+2)} \frac{\omega^{\frac{N+1}{2}+k}}{2(2 k+2) !} \ln \omega & =4^{k+\frac{1}{2}}\left(\mathcal{C}_{k}^{(\mathrm{o}, 3)}+\frac{\mathcal{C}_{k}^{(\mathrm{o}, 4)}}{2 k+2}\right), \\
-c_{\mathrm{o}, 1}^{(2 k+2)} \frac{\omega^{\frac{N+1}{2}}+k-1}{2(2 k+1) !} & =4^{k-1}(N+2 k+1)(N-3)\left(\mathrm{C}_{k}^{(\mathrm{o}, 3)}+\frac{\mathcal{C}_{k}^{(\mathrm{o}, 4)}}{2 k+2}\right),
\end{aligned}
$$

for the two unknowns $b_{1}^{(2 k+2)}$ and $c_{\mathrm{o}, 1}^{(2 k+2)}$. Solving Eqs. (6.16a) and 6.16b) for $c_{\mathrm{o}, 1}^{(i)}$ and $b_{1}^{(i)}$ gives

$$
c_{\mathrm{o}, 1}^{(i)}=0
$$

with $i=2,3, \cdots, N$,

$$
b_{1}^{(2 k+1)}=\mathrm{e}_{k}^{(\mathrm{o}, 2)}\left\{-2 k\left(\frac{N-1}{2}+k\right) !+\frac{2}{\sqrt{\pi}}\left(\frac{N}{2}+k\right) !_{3} F_{2}\left[\begin{array}{c}
\frac{1}{2}-k,-k,-\frac{1}{2} \\
\frac{1}{2},-k-\frac{N}{2}
\end{array} ; 1\right]\right\}
$$

with $k=1,2, \cdots,(N-1) / 2$, and

$$
b_{1}^{(2 k+2)}=\mathcal{C}_{k}^{(\mathrm{o}, 4)}\left\{\frac{-1}{2 k+2}\left(\frac{N+1}{2}+k\right) !+\frac{1}{\sqrt{\pi}}\left(\frac{N}{2}+k\right) !_{3} F_{2}\left[\begin{array}{c}
-\frac{1}{2}-k,-k, \frac{1}{2} \\
\frac{3}{2},-k-\frac{N}{2}
\end{array} ; 1\right]\right\}
$$

with $k=0,1, \cdots,(N-3) / 2$. Observe that the coefficients 6.17a) of the modified Bessel functions $K_{2}, K_{4}, \cdots, K_{N-1}$ are trivially independent of $\omega$, while the initial values (6.17b) and $(6.17 \mathrm{~g})$ of the $\omega=0$ recursion relation are independent of $\omega$ in view of Eqs. (6.12b) and (6.12d, respectively.

6.1.4. $\omega$ dependence of the recursion relation (6.2)

Equipped with the solutions (6.15) and (6.17) for the $\omega$ dependence of $b_{1}^{(i)}$ and $c_{\mathrm{o}, 1}^{(i)}$ with $i=$ $1,2, \cdots, N$, we can derive the $\omega$ dependence of the coefficients $b_{m}^{(i)}$ with $m=2,3, \cdots$ in the 
limit $1 \leq m \ll \omega^{-1}$ from Eq. 6.11). Insertion of Eq. 6.15) into the prefactors 6.12a) appearing in Eq. 6.11a) gives

$$
b_{m}^{(1)}=\frac{2 \mathcal{C}_{0}^{(\mathrm{o})}}{\ln \left(C_{0}^{(\mathrm{o})} \omega\right)} \sum_{l=0}^{m-1} \frac{\left(m-1-l+\frac{N-1}{2}\right) !}{(2 l+1)(m-1-l) !}+\mathcal{C}_{0}^{(\mathrm{o}, 2)} \frac{\left(m-1+\frac{N}{2}\right) !}{\left(m-\frac{1}{2}\right) !}
$$

with $\mathcal{C}_{0}^{(\mathrm{o})}$ some number that depends on $N$. Insertion of Eq. (6.17b) into the prefactor $6.12 \mathrm{~b}$ ) appearing in Eq. 6.11b) gives

$$
\begin{aligned}
b_{m}^{(2 k+1)}= & \mathcal{C}_{k}^{(\mathrm{o}, 2)} \frac{\left(m+k-1+\frac{N}{2}\right) !}{\left(m-\frac{1}{2}\right) !}{ }_{3} F_{2}\left[\begin{array}{l}
\frac{1}{2}-k,-k, \frac{1}{2}-m \\
\frac{1}{2}, 1-k-m-\frac{N}{2} ; 1
\end{array} ; 1\right] \\
& -\mathcal{C}_{k}^{(\mathrm{o}, 2)} 2 k \frac{\left(m+k-\frac{3}{2}+\frac{N}{2}\right) !}{(m-1) !}{ }_{3} F_{2}\left[\begin{array}{c}
\frac{1}{2}-k, 1-k, 1-m \\
\frac{3}{2}, \frac{3}{2}-k-m-\frac{N}{2} ; 1
\end{array} ;\right.
\end{aligned}
$$

with $k=1,2, \cdots,(N-1) / 2$. Insertion of Eq. (6.17g) into the prefactor $6.12 \mathrm{~d})$ appearing in Eq. 6.11c) gives

$$
\begin{aligned}
b_{m}^{(2 k+2)}= & \mathcal{C}_{k}^{(0,4)} \frac{\left(m+k-1+\frac{N}{2}\right) !}{\left(m-\frac{3}{2}\right) !}{ }_{3} F_{2}\left[\begin{array}{c}
-\frac{1}{2}-k,-k, \frac{3}{2}-m \\
\frac{3}{2}, 1-k-m-\frac{N}{2}
\end{array} ; 1\right] \\
& -\mathcal{C}_{k}^{(0,4)} \frac{1}{2 k+2} \frac{\left(m+k-\frac{1}{2}+\frac{N}{2}\right) !}{(m-1) !}{ }_{3} F_{2}\left[\begin{array}{c}
-\frac{1}{2}-k,-1-k, 1-m \\
\frac{1}{2}, \frac{1}{2}-k-m-\frac{N}{2}
\end{array} ; 1\right]
\end{aligned}
$$

with $k=0,1, \cdots,(N-3) / 2$.

In conclusion, the $\omega$ dependence of the solution to the recursion relation 6.2) follows from combining Eqs. 6.7), 6.8), 6.15), 6.17a) when $m \gg N$ together with Eq. 6.18) when $m \omega \ll$ 1. This gives

$$
\begin{aligned}
& b_{m}^{(1)}= \begin{cases}-\mathcal{C}_{0}^{(\mathrm{o})} \frac{2}{\ln \left(C_{0}^{(\mathrm{o})} \omega\right)} m^{\frac{N-1}{2}} K_{0}(2 \sqrt{m \omega}), & N \ll m, \\
\frac{2 \mathcal{C}_{0}^{(o)}}{\ln \left(C_{0}^{(\mathrm{o})} \omega\right)} \sum_{l=0}^{m-1} \frac{\left(m-1-l+\frac{N-1}{2}\right) !}{(2 l+1)(m-1-l) !}+\mathcal{C}_{0}^{(\mathrm{o}, 2)} \frac{\left(m-1+\frac{N}{2}\right) !}{\left(m-\frac{1}{2}\right) !}, & m \ll \omega^{-1},\end{cases} \\
& b_{m}^{(2 k+1)}= \begin{cases}0, & N \ll m, \\
6.18 \mathrm{~b}), & m \ll \omega^{-1},\end{cases} \\
& b_{m}^{\left(2 k^{\prime}+2\right)}= \begin{cases}0, & N \ll m, \\
6.18 \mathrm{~d}, & m \ll \omega^{-1},\end{cases}
\end{aligned}
$$

with $k=1,2, \cdots,(N-1) / 2$ and $k^{\prime}=0,1, \cdots,(N-3) / 2$. Equation (6.19) should be compared to Eq. (5.14). Observe that Eqs. (6.18b) and (6.18c) are independent of $\omega$.

\subsubsection{Leading energy dependence of the density of states}

We are ready to extract the leading behavior of the average density of states $\nu(\varepsilon)$, Eq. (3.25), for asymptotically small energies $\varepsilon$. The density of states in the long wire limit Eq. (3.24) is given by 


$$
\nu(\varepsilon)=\lim _{\omega \rightarrow-i \varepsilon} \pi^{-1} \operatorname{Re}_{\mathrm{L}}\left\langle\varphi_{0}|(\bar{B}-B)| \varphi_{0}\right\rangle_{\mathrm{R}} .
$$

with the left and right ground states $(4.10 \mathrm{a})$ and $(4.10 \mathrm{~b})$. The normalizations $(4.8)$ and $(4.11)$, the biorthogonal relations (4.9), and the identities

$$
\begin{aligned}
& (\bar{B}-B)|0\rangle=N|0\rangle, \\
& (\bar{B}-B)|m\rangle_{\mathrm{R}}^{(2 n+1)}=(N-4 n-1)|m\rangle_{\mathrm{R}}^{(2 n+1)}+|m\rangle_{\mathrm{L}}^{(2 n+1)}, \\
& (\bar{B}-B)|m\rangle_{\mathrm{R}}^{(2 n+2)}=(N-4 n-3)|m\rangle_{\mathrm{R}}^{(2 n+2)}-|m\rangle_{\mathrm{L}}^{(2 n+2)},
\end{aligned}
$$

deliver the expectation value

$$
\begin{aligned}
\mathrm{L}\left\langle\varphi_{0}|(\bar{B}-B)| \varphi_{0}\right\rangle_{\mathrm{R}}= & N+\sum_{m=1}^{\infty} \sum_{n=0}^{(N-1) / 2} \mathcal{N}_{m, n}^{(1)}\left(a_{m}^{(2 n+1)}\right)^{2}-\sum_{m=1}^{\infty} \sum_{n=0}^{(N-3) / 2} \mathcal{N}_{m, n}^{(2)}\left(a_{m}^{(2 n+2)}\right)^{2} \\
= & N+\sum_{m=1}^{\infty} \sum_{n=0}^{(N-1) / 2} \mathcal{N}_{m, n}^{(1)}\left(\sum_{k=0}^{(N-1) / 2}\left[\mathcal{N}_{\mathrm{o} ; N, 1}^{-1}\right]_{k}^{n} b_{m}^{(2 k+1)}\right)^{2} \\
& -\sum_{m=1}^{\infty} \sum_{n=0}^{(N-3) / 2} \mathcal{N}_{m, n}^{(2)}\left(\sum_{k=0}^{(N-3) / 2}\left[\mathcal{M}_{\mathrm{o} ; N, 2}^{-1}\right]_{k}^{n} b_{m}^{(2 k+2)}\right)^{2} .
\end{aligned}
$$

The matrices $\mathcal{M}_{\mathrm{o} ; N, 1}$ and $\mathcal{M}_{\mathrm{o} ; N, 2}$ are given in Eq. 6.1], while the normalization factors $\mathcal{N}_{m, n}^{(1)}$ and $\mathcal{N}_{m, n}^{(2)}$ are given by Eq. 4.8). In view of Eq. (6.19), it is necessary to break the sum over $m$ in Eq. 6.22 into two parts separated by the integer $m_{0}$, with $N \ll m_{0} \ll \omega^{-1}$. We choose $m_{0}$ to be some number independent of $\omega$. With this choice and for sufficiently small $\omega$, we find that the sum over $m$ in Eq. 6.22 is dominated by the contributions from $b_{m}^{(1)}$ with $m>m_{0}$, i.e., by the contributions from the first line in Eq. 6.19),

$$
{ }_{\mathrm{L}}\left\langle\varphi_{0}|(\bar{B}-B)| \varphi_{0}\right\rangle_{\mathrm{R}} \approx \int_{m_{0}}^{\infty} d m \sum_{n=0}^{(N-1) / 2} \mathcal{N}_{m, n}^{(1)}\left(\left[\mathcal{M}_{\mathrm{o} ; N, 1}^{-1}\right]_{0}^{n} b_{m}^{(1)}\right)^{2} .
$$

By inserting the solution for $b_{m}^{(1)}$ from Eq. (6.19) we obtain

$$
{ }_{\mathrm{L}}\left\langle\varphi_{0}|(\bar{B}-B)| \varphi_{0}\right\rangle_{\mathrm{R}} \approx \frac{4\left(\mathcal{C}_{0}^{(\mathrm{o})}\right)^{2}}{\ln ^{2}\left(C_{0}^{(\mathrm{o})} \omega\right)} \int_{m_{0}}^{\infty} d m \mathcal{S}_{N}^{(\mathrm{o})}(m) m^{N-1} K_{0}^{2}(2 \sqrt{m \omega}),
$$

with the combinatorial factor

$$
\mathcal{S}_{N}^{(\mathrm{o})}(m):=\sum_{n=0}^{(N-1) / 2} \mathcal{N}_{m, n}^{(1)}\left(\left[\mathcal{M}_{\mathrm{o} ; N, 1}^{-1}\right]_{0}^{n}\right)^{2} .
$$

Using the substitution $x^{2}=4 m \omega$, the integral transforms into

$$
{ }_{\mathrm{L}}\left\langle\varphi_{0}|(\bar{B}-B)| \varphi_{0}\right\rangle_{\mathrm{R}} \approx \frac{4\left(\mathcal{C}_{0}^{(\mathrm{o})}\right)^{2} \omega^{-N}}{\ln ^{2}\left(C_{0}^{(\mathrm{o})} \omega\right)} \int_{x_{0}}^{\infty} d x \mathcal{S}_{N}^{(\mathrm{o})}\left(\frac{x^{2}}{4 \omega}\right)\left(\frac{x}{2}\right)^{2 N-1} K_{0}^{2}(x),
$$

with $x_{0}:=2 \sqrt{m_{0} \omega}$. Let us now expand $\mathcal{S}_{N}^{(\text {o) }}$ in small $\omega$ (i.e., large argument) 


$$
\mathcal{S}_{N}^{(\mathrm{o})}\left(\frac{x^{2}}{4 \omega}\right) \approx \mathcal{S}_{N}^{(\mathrm{o}, 1)} \times\left(\frac{2 \omega}{x^{2}}\right)^{N-1}+\mathcal{O}\left[\left(\frac{2 \omega}{x^{2}}\right)^{N}\right],
$$

where $\mathcal{S}_{N}^{(0,1)}$ is some number that only depends on $N$. In order to derive the asymptotic density of states, it is sufficient to keep the leading $\omega$ dependence in Eq. (6.26). This gives

$$
{ }_{\mathrm{L}}\left\langle\varphi_{0}|(\bar{B}-B)| \varphi_{0}\right\rangle_{\mathrm{R}} \approx \frac{4\left(\mathcal{C}_{0}^{(\mathrm{o})}\right)^{2} 2^{-N} \omega^{-1}}{\ln ^{2}\left(C_{0}^{(\mathrm{o})} \omega\right)} \mathcal{S}_{N}^{(\mathrm{o}, 1)} \int_{x_{0}}^{\infty} d x x K_{0}^{2}(x) .
$$

Combining Eq. 6.27) and (6.20), we find that the density of states, for asymptotically small energies, is given by

$$
\nu(\varepsilon) \propto \lim _{\omega \rightarrow-i \varepsilon} \operatorname{Re} \frac{1}{\omega \ln ^{2}\left(C_{0}^{(\mathrm{o})} \omega\right)} \sim \frac{1}{\varepsilon\left|\ln ^{3} \varepsilon\right|} .
$$

Recalling that $\varepsilon$ is measured in units of the disorder strength, we have recovered Eq. (1.1) when the number of channels $N$ is odd.

\subsection{Density of states for an even number $N$ of channels}

The calculation of the density of states for an even number $N$ of channels follows along the lines of the $N=2$ case with the caveat that for $N>2$ the recursion relations 4.13 no longer decouple. This difference complicates the calculation considerably. To overcome this difficulty we introduce a linear transformation of the coefficients $a_{m}^{(n)}$ that approximately decouples the recursion relations (4.13) in the two regimes $m \gg N$ and $m \ll \omega^{-1}$. That is, we introduce

$$
\begin{aligned}
b_{m}^{(2 k+1)}=\sum_{n=0}^{N / 2-1}\left[\mathcal{M}_{\mathrm{e} ; N, 1}\right]_{n}^{k} a_{m}^{(2 n+1)}, & k=0,1, \cdots, \frac{N}{2}-1, \\
b_{m}^{(2 k+2)}=\sum_{n=0}^{N / 2-1}\left[\mathcal{M}_{\mathrm{e} ; N, 2}\right]_{n}^{k} a_{m}^{(2 n+2)}, & k=0,1, \cdots, \frac{N}{2}-1,
\end{aligned}
$$

with $m=1,2,3, \cdots$ and the transformation matrices

$$
\begin{aligned}
& {\left[\mathcal{M}_{\mathrm{e} ; N, 1}\right]_{n}^{k}:=(-1)^{n} \frac{(2 n) !(N-1-2 n) !\left(\frac{N-2}{2}\right) !}{n !\left(\frac{N-2}{2}-n\right) !(N-1) !}{ }_{3} F_{2}\left[\begin{array}{c}
-k, k+1,-n \\
\frac{1}{2}, \frac{2-N}{2} ; 1
\end{array}\right],} \\
& {\left[\mathcal{M}_{\mathrm{e} ; N, 2}\right]_{n}^{k}:=(-1)^{n} \frac{(2 n+1) !(N-2-2 n) !\left(\frac{N-2}{2}\right) !}{n !\left(\frac{N-2}{2}-n\right) !(N-2) !}{ }_{3} F_{2}\left[\begin{array}{c}
-k, k+1,-n \\
\frac{3}{2}, \frac{2-N}{2} ; 1
\end{array}\right] .}
\end{aligned}
$$

Here, the symbol ${ }_{3} F_{2}$ denotes a generalized hypergeometric function (see for example Ref. [93]). It is also possible to express the $a_{m}^{(n)}$ 's in terms of the $b_{m}^{(k)}$ 's. Indeed, with the help of identities (B.5a) and (B.5b), inverting the linear relation (6.29a), i.e., for any $m=1,2,3, \cdots$, 


$$
\begin{array}{ll}
a_{m}^{(2 n+1)}=\sum_{k=0}^{N / 2-1}\left[\mathcal{M}_{\mathrm{e} ; N, 1}^{-1}\right]_{k}^{n} b_{m}^{(2 k+1)}, & n=0,1, \cdots, \frac{N}{2}-1, \\
a_{m}^{(2 n+2)}=\sum_{k=0}^{N / 2-1}\left[\mathcal{M}_{\mathrm{e} ; N, 2}^{-1}\right]_{k}^{n} b_{m}^{(2 k+2)}, & n=0,1, \cdots, \frac{N}{2}-1,
\end{array}
$$

can be done with the inverses

$$
\begin{aligned}
& {\left[\mathcal{M}_{\mathrm{e} ; N, 1}^{-1}\right]_{k}^{n}=\frac{\frac{2^{2-N}}{(2 n) !}[(N-1) !]^{2}}{(N-1-2 n) !\left(\frac{N-2}{2}-k\right) !\left(\frac{N}{2}+k\right) !}{ }_{3} F_{2}\left[\begin{array}{c}
-k-\frac{N}{2}, k+\frac{2-N}{2},-n \\
\frac{1}{2}-\frac{N}{2}, \frac{2-N}{2}
\end{array}\right],} \\
& {\left[\mathcal{M}_{\mathrm{e} ; N, 2}^{-1}\right]_{k}^{n}=\frac{\frac{2^{2-N}}{(2 n+1) !}[(N-2) !]^{2}(2 k+1)^{2}}{(N-2-2 n) !\left(\frac{N-2}{2}-k\right) !\left(\frac{N}{2}+k\right) !}{ }_{3} F_{2}\left[\begin{array}{c}
-k-\frac{N}{2}, k+\frac{2-N}{2},-n \\
1-\frac{N}{2}, \frac{3-N}{2} ; 1
\end{array}\right],}
\end{aligned}
$$

of the transformation matrices $6.29 \mathrm{~b}$. With these definitions, we are going to rewrite the recursion relations $(4.13$ ) in a form that decouples both in the limit $\omega=0$ and in the large $m$ limit.

To see this, we first make use of identities $(\mathrm{B} .3 \mathrm{~d})$ and $(\mathrm{B.3 \textrm {d }})$ to combine Eqs. (4.13) and $(6.29)$ into the recursion relations

$$
\begin{aligned}
4 m \omega b_{m}^{(2 k+1)}= & \{-4 m(2 m-1+N)+[N-2-4 k(k+1)]\} b_{m}^{(2 k+1)} \\
& +2 m(2 m+1) b_{m+1}^{(2 k+1)} \\
& +(2 m-2+N)(2 m-1+N) b_{m-1}^{(2 k+1)} \\
& -4 \omega \sum_{n=0}^{N / 2-1} n\left[\mathcal{M}_{\mathrm{e} ; N, 1}\right]_{n}^{k} a_{m}^{(2 n+1)}
\end{aligned}
$$

and

$$
\begin{aligned}
4 m \omega b_{m}^{(2 k+2)}= & \{-4 m(2 m-3+N)+[3 N-6-4 k(k+1)]\} b_{m}^{(2 k+2)} \\
& +2 m(2 m-1) b_{m+1}^{(2 k+2)} \\
& +(2 m-3+N)(2 m-2+N) b_{m-1}^{(2 k+2)} \\
& -4 \omega \sum_{n=0}^{N / 2-1} n\left[\mathcal{M}_{\mathrm{e} ; N, 2}\right]_{n}^{k} a_{m}^{(2 n+2)}
\end{aligned}
$$

with $k=0,1, \cdots,(N / 2)-1$ and $m=2,3, \cdots$. Here, the initial conditions are

$$
\begin{aligned}
4 \omega b_{1}^{(2 k+1)}= & +\sqrt{2} N(N+1)-[3 N+6+4 k(k+1)] b_{1}^{(2 k+1)}+6 b_{2}^{(2 k+1)} \\
& -4 \omega \sum_{n=0}^{N / 2-1} n\left[\mathcal{M}_{\mathrm{e} ; N, 1}\right]_{n}^{k} a_{1}^{(2 n+1)}
\end{aligned}
$$

with $k=0,1, \cdots,(N / 2)-1$ and

$$
\begin{aligned}
4 \omega b_{1}^{(2 k+2)}= & -\sqrt{2} N(N-1)-[N+2+4 k(k+1)] b_{1}^{(2 k+2)}+2 b_{2}^{(2 k+2)} \\
& -4 \omega \sum_{n=0}^{N / 2-1} n\left[\mathcal{M}_{\mathrm{e} ; N, 2}\right]_{n}^{k} a_{1}^{(2 n+2)}
\end{aligned}
$$


with $k=0,1,2, \cdots,(N / 2)-1$. We aim at a solution of Eq. (6.30) for asymptotically small frequency $\omega$. As in Sec. 5.2, our strategy is to solve Eq. (6.30) in the two limits $N \ll m$ and $m \ll \omega^{-1}$. In the former limit, $m \gg N$, we treat $m$ as a continuous variable. Then, Eq. (6.30) decouples, provided we assume that $a_{m}^{(n)}$ decays rapidly for $m \gg N$. In the other limit, $m \ll$ $\omega^{-1}$, we can neglect $\omega$ in Eq. (6.30), which again decouples Eq. 6.30). In this way, it is possible to find approximate solutions in the two regions $N \ll m$ and $m \ll \omega^{-1}$ that are uniquely fixed up to some multiplicative coefficients and initial values, respectively. For asymptotically small $\omega$, the overlap between these two regions $N \ll m \ll \omega^{-1}$ is large. We can then match the two approximate solutions in the overlapping region. This gives a unique and approximate solution for the ground state wave function, which in turn determines the density of states $\sqrt{3.25}$ ) in the long wire limit.

\subsubsection{Solution when $m \gg N$}

First, we treat the limit $m \gg N$. If we assume that $a_{m}^{(n)}$ decays rapidly for large $m$, we can drop the last line on the right-hand side of Eqs. (6.30a) and (6.30b), respectively. Consequently, in terms of the finite differences

$$
\Delta b_{m}^{(i)}:=b_{m+1}^{(i)}-b_{m}^{(i)}, \quad \quad \Delta^{2} b_{m}^{(i)}:=b_{m+2}^{(i)}-2 b_{m+1}^{(i)}+b_{m}^{(i)},
$$

with $i=1, \cdots, N$, the recursion relation 6.30) reads

$$
\begin{aligned}
(m+1) 4 \omega\left(\Delta b_{m}^{(2 k+1)}+b_{m}^{(2 k+1)}\right)= & -2(2 m+2)(N-2) \Delta b_{m}^{(2 k+1)} \\
& +(2 m+2)(2 m+3) \Delta^{2} b_{m}^{(2 k+1)} \\
& +(N-1)(N-2) b_{m}^{(2 k+1)} \\
& +[N-2-4 k(k+1)]\left(\Delta b_{m}^{(2 k+1)}+b_{m}^{(2 k+1)}\right)
\end{aligned}
$$

with $k=0,1, \cdots,(N / 2)-1$ and

$$
\begin{aligned}
(m+1) 4 \omega\left(\Delta b_{m}^{(2 k+2)}+b_{m}^{(2 k+2)}\right)= & -2(2 m+2)(N-2) \Delta b_{m}^{(2 k+2)} \\
& +(2 m+2)(2 m+1) \Delta^{2} b_{m}^{(2 k+2)} \\
& +(N-2)(N-3) b_{m}^{(2 k+2)} \\
& +[3 N-6-4 k(k+1)]\left(\Delta b_{m}^{(2 k+2)}+b_{m}^{(2 k+2)}\right)
\end{aligned}
$$

with $k=0,1, \cdots,(N / 2)-1$. Observe that Eqs. 6.32a) and (6.32b) only differ from Eqs. 6.4a) and (6.4b) through the coefficients $[N-2-4 k(k+1)]$ and $[3 N-6-4 k(k-1)]$, respectively. In the limit $m \gg N$, we can neglect terms of order $N$ compared to $m$ and replace finite differences by derivatives. In place of Eq. 6.32 and if we assume that $b_{m}^{(i)} \rightarrow b^{(i)}(m)$ with $i=1,2, \cdots, N$ is slowly varying, we get

$$
4 m \omega b^{(2 k+1)}=-4 m(N-2) \frac{d b^{(2 k+1)}}{d m}+4 m^{2} \frac{d^{2} b^{(2 k+1)}}{d m^{2}}+\left[(N-1)^{2}-(2 k+1)^{2}\right] b^{(2 k+1)}
$$

and

$$
4 m \omega b^{(2 k+2)}=-4 m(N-2) \frac{d b^{(2 k+2)}}{d m}+4 m^{2} \frac{d^{2} b^{(2 k+2)}}{d m^{2}}+\left[(N-1)^{2}-(2 k+1)^{2}\right] b^{(2 k+2)}
$$


with $k=0,1, \cdots,(N / 2)-1$. By use of the substitution

$$
x^{2}:=4 \omega m
$$

whereby

$$
\frac{x^{2}}{4 \omega} \gg N
$$

we find that the solutions to Eq. 6.33) are given by the linear combinations

$$
b^{(2 k+1)}(x)=c_{\mathrm{e}, 1}^{(2 k+1)}\left(\frac{x}{2}\right)^{N-1} K_{2 k+1}(x)+c_{\mathrm{e}, 2}^{(2 k+1)}\left(\frac{x}{2}\right)^{N-1} I_{2 k+1}(x)
$$

and

$$
b^{(2 k+2)}(x)=c_{\mathrm{e}, 1}^{(2 k+2)}\left(\frac{x}{2}\right)^{N-1} K_{2 k+1}(x)+c_{\mathrm{e}, 2}^{(2 k+2)}\left(\frac{x}{2}\right)^{N-1} I_{2 k+1}(x)
$$

with $k=0,1, \cdots,(N / 2)-1$, of modified Bessel functions $K_{2 k+1}$ and $I_{2 k+1}$. We shall demand that $b_{m}^{(i)}$ with $i=1, \cdots, N$ decay to zero as $m \rightarrow \infty$, i.e., we must set

$$
c_{\mathrm{e}, 2}^{(i)}=0
$$

with $i=1, \cdots, N$ in Eq. (6.35). The remaining $N$ coefficients $c_{\mathrm{e}, 1}^{(i)}$ with $i=1, \cdots, N$ of the modified Bessel functions $K_{1}, K_{3}, K_{5}, \cdots, K_{N-1}$ are fixed by matching solutions (6.35) to the solutions in the $m \ll \omega^{-1}$ region. Thereto, we need to extract the terms that are of order $x^{N+2 k}$ and $x^{N+2 k-2}$ from the expansion of Eq. (6.35) when $x$ small (see chapter 9.6 in Ref. 92]). This gives

$$
\begin{aligned}
b^{(2 k+1)}(x) & \sim c_{\mathrm{e}, 1}^{(2 k+1)}\left[\frac{(x / 2)^{N+2 k}}{2(2 k+1) !}\left(\gamma+\ln \frac{x^{2}}{4}-\Psi(2 k+2)\right)+\frac{(x / 2)^{N+2 k-2}}{2(2 k) !}\right] \\
& =c_{\mathrm{e}, 1}^{(2 k+1)}\left[\frac{(m \omega)^{\frac{N}{2}+k}}{2(2 k+1) !} \ln \left(C_{k}^{(\mathrm{e})} m \omega\right)+\frac{(m \omega)^{\frac{N}{2}+k-1}}{2(2 k) !}\right]
\end{aligned}
$$

and

$$
\begin{aligned}
b^{(2 k+2)}(x) & \sim c_{\mathrm{e}, 1}^{(2 k+2)}\left[\frac{(x / 2)^{N+2 k}}{2(2 k+1) !}\left(\gamma+\ln \frac{x^{2}}{4}-\Psi(2 k+2)\right)+\frac{(x / 2)^{N+2 k-2}}{2(2 k) !}\right] \\
& =c_{\mathrm{e}, 1}^{(2 k+2)}\left[\frac{(m \omega)^{\frac{N}{2}+k}}{2(2 k+1) !} \ln \left(C_{k}^{(\mathrm{e})} m \omega\right)+\frac{(m \omega)^{\frac{N}{2}+k-1}}{2(2 k) !}\right]
\end{aligned}
$$

with $k=0,1, \cdots,(N / 2)-1$ when $x \ll 1$, and where

$$
C_{k}^{(\mathrm{e})}:=\exp (\gamma-\Psi(2 k+2)) \text {. }
$$

\subsubsection{Solution when $1 \leq m \ll \omega^{-1}$}

Second, we treat the limit $1 \leq m \ll \omega^{-1}$, in which case we can neglect the $\omega$ terms in Eq. 6.30). In doing so, Eq. (6.30) becomes

$$
\begin{aligned}
0=\{ & -4 m(2 m-1+N)+[N-2-4 k(k+1)]\} b_{m}^{(2 k+1)}+2 m(2 m+1) b_{m+1}^{(2 k+1)} \\
& +(2 m-2+N)(2 m-1+N) b_{m-1}^{(2 k+1)}
\end{aligned}
$$


and

$$
\begin{aligned}
0= & \{-4 m(2 m-3+N)+[3 N-6-4 k(k+1)]\} b_{m}^{(2 k+2)}+2 m(2 m-1) b_{m+1}^{(2 k+2)} \\
& +(2 m-3+N)(2 m-2+N) b_{m-1}^{(2 k+2)}
\end{aligned}
$$

with $k=0,1, \cdots,(N / 2)-1$ and $m=2,3, \cdots$, together with the initial conditions

$$
0=+\sqrt{2} N(N+1)-[3 N+6+4 k(k+1)] b_{1}^{(2 k+1)}+6 b_{2}^{(2 k+1)}
$$

and

$$
0=-\sqrt{2} N(N-1)-[N+2+4 k(k+1)] b_{1}^{(2 k+2)}+2 b_{2}^{(2 k+2)} .
$$

The solution to the recursion relation 6.38 can be expressed in terms of generalized hypergeometric functions

$$
\begin{aligned}
b_{m}^{(2 k+1)}= & \left.\mathcal{C}_{k}^{(\mathrm{e}, 1)} \frac{\left(m+k-1+\frac{N}{2}\right) !}{(m-1) !}{ }_{3} F_{2}\left[\begin{array}{c}
-k, \frac{1}{2}-k, 1-m \\
\frac{3}{2}, 1-k-m-\frac{N}{2}
\end{array}\right] 1\right] \\
& +\mathcal{C}_{k}^{(\mathrm{e}, 2)} \frac{\left(m+k-\frac{1}{2}+\frac{N}{2}\right) !}{\left(m-\frac{1}{2}\right) !}{ }_{3} F_{2}\left[\begin{array}{c}
-k,-\frac{1}{2}-k, \frac{1}{2}-m \\
\frac{1}{2}, \frac{1}{2}-k-m-\frac{N}{2}
\end{array} ; 1\right]
\end{aligned}
$$

and

$$
\begin{aligned}
b_{m}^{(2 k+2)}= & \mathcal{C}_{k}^{(\mathrm{e}, 3)} \frac{\left(m+k-1+\frac{N}{2}\right) !}{(m-1) !}{ }_{3} F_{2}\left[\begin{array}{c}
-k,-\frac{1}{2}-k, 1-m \\
\frac{1}{2}, 1-k-m-\frac{N}{2}
\end{array} ; 1\right] \\
& +\mathcal{C}_{k}^{(\mathrm{e}, 4)} \frac{\left(m+k-\frac{3}{2}+\frac{N}{2}\right) !}{\left(m-\frac{3}{2}\right) !}{ }_{3} F_{2}\left[\begin{array}{c}
-k, \frac{1}{2}-k, \frac{3}{2}-m \\
\frac{3}{2}, \frac{3}{2}-k-m-\frac{N}{2}
\end{array} ; 1\right]
\end{aligned}
$$

with $k=0,1, \cdots,(N / 2)-1$ and $m=2,3, \cdots$. The prefactors $\mathcal{C}_{k}^{(\mathrm{e}, i)} i=1,2,3,4$ to the hypergeometric function ${ }_{3} F_{2}$ are determined by the initial conditions (6.38c) and (6.38d) through

$$
\begin{aligned}
\mathcal{C}_{k}^{(\mathrm{e}, 1)} & =\frac{1}{\left(k+\frac{N}{2}\right) !}\left\{b_{1}^{(2 k+1)}-\mathcal{C}_{k}^{(\mathrm{e}, 2)} \frac{2}{\sqrt{\pi}}\left(k+\frac{N+1}{2}\right) !{ }_{3} F_{2}\left[\begin{array}{c}
-k,-\frac{1}{2}-k,-\frac{1}{2} \\
\frac{1}{2},-\frac{1}{2}-k-\frac{N}{2}
\end{array} ; 1\right]\right\}, \\
\mathcal{C}_{k}^{(\mathrm{e}, 2)} & =\sqrt{\pi} 2^{k+\frac{1}{2}} \frac{(N-2 k-2) ! !}{(N-2) ! !\left(\frac{N-1}{2}\right) !},
\end{aligned}
$$

and

$$
\begin{aligned}
& \mathcal{C}_{k}^{(\mathrm{e}, 3)}=\frac{1}{\left(k+\frac{N}{2}\right) !}\left\{b_{1}^{(2 k+2)}-\mathcal{C}_{k}^{(\mathrm{e}, 4)} \frac{1}{\sqrt{\pi}}\left(k+\frac{N-1}{2}\right) !{ }_{3} F_{2}\left[\begin{array}{c}
-k, \frac{1}{2}-k, \frac{1}{2} \\
\frac{3}{2}, \frac{1}{2}-k-\frac{N}{2}
\end{array} ; 1\right]\right\}, \\
& \mathcal{C}_{k}^{(\mathrm{e}, 4)}=\sqrt{\pi} 2^{k+\frac{3}{2}} \frac{(N-2 k-2) ! !}{(N-2) ! !\left(\frac{N-3}{2}\right) !}
\end{aligned}
$$

with $k=0,1, \cdots,(N / 2)-1$. It is interesting to note that the explicit $k$ dependences on the righthand sides of Eqs. (6.39a and (6.39b) are related to those on the right-hand sides of Eqs. 6.11b) and $(6.11 \mathrm{~d})$ by letting $k \rightarrow k-(1 / 2)$ and $k \rightarrow k+(1 / 2)$, respectively. 
In order to match this solution when $1 \leq m \ll 1 / \omega$ to the solution when $N \ll m$, we need the leading and subleading terms of the large $m$ behavior of $b_{m}^{(2 k+1)}$ and $b_{m}^{(2 k+2)}$. For $b_{m}^{(1)}$ and $b_{m}^{(2)}$, we have

$$
\begin{aligned}
& b_{m}^{(1)} \simeq\left(\mathrm{C}_{0}^{(\mathrm{e}, 1)}+\mathrm{C}_{0}^{(\mathrm{e}, 2)}\right) m^{\frac{N}{2}}+\frac{N}{8}\left((N-2) \mathrm{C}_{0}^{(\mathrm{e}, 1)}+N \mathrm{C}_{0}^{(\mathrm{e}, 2)}\right) m^{\frac{N}{2}-1} \\
& b_{m}^{(2)} \simeq\left(\mathrm{C}_{0}^{(\mathrm{e}, 3)}+\mathrm{C}_{0}^{(\mathrm{e}, 4)}\right) m^{\frac{N}{2}}+\frac{N}{8}\left((N-2) \mathrm{C}_{0}^{(\mathrm{e}, 3)}+(N-4) \mathrm{C}_{0}^{(\mathrm{e}, 4)}\right) m^{\frac{N}{2}-1},
\end{aligned}
$$

to leading and subleading order in $m$, respectively. In general we have for the odd-numbered coefficients

$$
\begin{aligned}
b_{m}^{(2 k+1)} \simeq & 4^{k}\left(\frac{\mathcal{C}_{k}^{(\mathrm{e}, 1)}}{2 k+1}+\mathcal{C}_{k}^{(\mathrm{e}, 2)}\right) m^{\frac{N}{2}+k} \\
& +4^{k-\frac{3}{2}}(N+2 k)(N-1)\left(\frac{\mathcal{C}_{k}^{(\mathrm{e}, 1)}}{2 k+1}+\mathcal{C}_{k}^{(\mathrm{e}, 2)}\right) m^{\frac{N}{2}+k-1}
\end{aligned}
$$

and for the even-numbered coefficients

$$
\begin{aligned}
b_{m}^{(2 k+2)} \simeq & 4^{k}\left(\mathcal{C}_{k}^{(\mathrm{e}, 3)}+\frac{\mathcal{C}_{k}^{(\mathrm{e}, 4)}}{2 k+1}\right) m^{\frac{N}{2}+k} \\
& +4^{k-\frac{3}{2}}(N+2 k)(N-3)\left(\mathcal{C}_{k}^{(\mathrm{e}, 3)}+\frac{\mathcal{C}_{k}^{(\mathrm{e}, 4)}}{2 k+1}\right) m^{\frac{N}{2}+k-1}
\end{aligned}
$$

with $k=1, \cdots,(N / 2)-1$, to leading and subleading order in $m$. We emphasize that these two formula are only valid for $k \geq 1$. Observe that the large $m$ subleading behavior of $b_{m}^{(1)}$ and $b_{m}^{(2)}$ differ from those of $b_{m}^{(2 k+1)}$ and $b_{m}^{(2 k+2)}$ with $k \geq 1$, respectively. We note again that the explicit $k$ dependences on the right-hand sides of Eqs. (6.41c) and 6.41d) are related to those on the right-hand sides of Eqs. (6.13b) and (6.13c) by letting $k \rightarrow k-(1 / 2)$ and $k \rightarrow k+(1 / 2)$, respectively.

\subsubsection{Matching solutions when $N \ll m \ll \omega^{-1}$}

Having solved the recursion relations (6.30) in the two limits $N \ll m$ and $1 \ll m \ll \omega^{-1}$, we are going to match the $m=x^{2} /(4 \omega)$ dependences of the solutions 6.37 ) and 6.41 in the overlapping region $N \ll m \ll \omega^{-1}$, where both solutions are valid. We start with the coefficients $b_{m}^{(1)}$ and $b_{m}^{(2)}$. Matching Eq. (6.37a) when $k=0$ with Eq. 6.41a) gives two equations in the two unknowns $b_{1}^{(1)}$ and $c_{\mathrm{e}, 1}^{(1)}$ (whereby we neglect $\ln m$ and $\ln C_{k}^{(\mathrm{e})}$ compared to $\ln \omega$ )

$$
\begin{aligned}
c_{\mathrm{e}, 1}^{(1)} \frac{1}{2} \omega^{\frac{N}{2}} \ln \omega & =\mathcal{C}_{0}^{(\mathrm{e}, 1)}+\mathcal{C}_{0}^{(\mathrm{e}, 2)}, \\
c_{\mathrm{e}, 1}^{(1)} \frac{1}{2} \omega^{\frac{N}{2}-1} & =\frac{N}{8}\left[(N-2) \mathcal{C}_{0}^{(\mathrm{e}, 1)}+N \mathcal{C}_{0}^{(\mathrm{e}, 2)}\right] .
\end{aligned}
$$

Matching Eq. (6.37b) when $k=0$ with Eq. (5.41b) gives two equations in the two unknowns $b_{1}^{(2)}$ and $c_{\mathrm{e}, 1}^{(2)}$,

$$
\begin{aligned}
c_{\mathrm{e}, 1}^{(2)} \frac{1}{2} \omega^{\frac{N}{2}} \ln \omega & =\mathcal{C}_{0}^{(\mathrm{e}, 3)}+\mathcal{C}_{0}^{(\mathrm{e}, 4)}, \\
c_{\mathrm{e}, 1}^{(2)} \frac{1}{2} \omega^{\frac{N}{2}-1} & =\frac{N}{8}\left[(N-2) \mathcal{C}_{0}^{(\mathrm{e}, 3)}+(N-4) \mathcal{C}_{0}^{(\mathrm{e}, 4)}\right] .
\end{aligned}
$$


Solving for $b_{1}^{(1)}, b_{1}^{(2)}, c_{\mathrm{e}, 1}^{(1)}$, and $c_{\mathrm{e}, 1}^{(2)}$, we determine their $\omega$ dependences,

$$
\begin{aligned}
c_{\mathrm{e}, 1}^{(1)} & =\frac{+4 \mathrm{C}_{0}^{(\mathrm{e}, 2)} N \omega^{1-\frac{N}{2}}}{8-N(N-2) \omega \ln \omega}, \\
c_{\mathrm{e}, 1}^{(2)} & =\frac{-4 \mathrm{C}_{0}^{(\mathrm{e}, 4)} N \omega^{1-\frac{N}{2}}}{8-N(N-2) \omega \ln \omega},
\end{aligned}
$$

and

$$
\begin{aligned}
& b_{1}^{(1)}=\sqrt{2}(N+1)-\frac{\mathcal{C}_{0}^{(\mathrm{e}, 2)}\left(\frac{N}{2}\right) !\left[8-N^{2} \omega \ln \omega\right]}{8-N(N-2) \omega \ln \omega}, \\
& b_{1}^{(2)}=\sqrt{2}(N-1)-\frac{\mathrm{C}_{0}^{\mathrm{e}, 4)}\left(\frac{N}{2}\right) ![8-N(N-4) \omega \ln \omega]}{8-N(N-2) \omega \ln \omega},
\end{aligned}
$$

respectively. For $k \geq 1$, matching Eq. 6.37a) with Eq. (6.41d) gives two equations for the two unknowns $b_{1}^{(2 k+1)}$ and $c_{\mathrm{e}, 1}^{(2 k+1)}$ (whereby we neglect $\ln m$ and $\ln C_{k}^{(\mathrm{e})}$ compared to $\ln \omega$ )

$$
\begin{aligned}
c_{\mathrm{e}, 1}^{(2 k+1)} \frac{\omega^{\frac{N}{2}+k}}{2(2 k+1) !} \ln \omega & =4^{k}\left(\frac{\mathcal{C}_{k}^{(\mathrm{e}, 1)}}{2 k+1}+\mathcal{C}_{k}^{(\mathrm{e}, 2)}\right), \\
c_{\mathrm{e}, 1}^{(2 k+1)} \frac{\omega^{\frac{N}{2}+k-1}}{2(2 k) !} & =4^{k-\frac{3}{2}}(N+2 k)(N-1)\left(\frac{\mathcal{C}_{k}^{(\mathrm{e}, 1)}}{2 k+1}+\mathcal{C}_{k}^{(\mathrm{e}, 2)}\right),
\end{aligned}
$$

while matching Eq. 6.37b) with Eq. (6.41d) gives two equations for the two unknowns $b_{1}^{(2 k+2)}$ and $c_{\mathrm{e}, 1}^{(2 k+2)}$

$$
\begin{aligned}
c_{\mathrm{e}, 1}^{(2 k+2)} \frac{\omega^{\frac{N}{2}+k}}{2(2 k+1) !} \ln \omega & =4^{k}\left(\mathcal{C}_{k}^{(\mathrm{e}, 3)}+\frac{\mathcal{C}_{k}^{(\mathrm{e}, 4)}}{2 k+1}\right), \\
c_{\mathrm{e}, 1}^{(2 k+2)} \frac{\omega^{\frac{N}{2}+k-1}}{2(2 k) !} & =4^{k-\frac{3}{2}}(N+2 k)(N-3)\left(\mathcal{C}_{k}^{(\mathrm{e}, 3)}+\frac{\mathcal{C}_{k}^{(\mathrm{e}, 4)}}{2 k+1}\right),
\end{aligned}
$$

with $k=1,2, \cdots,(N / 2)-1$. Solving for $b_{1}^{(i)}$ and $c_{\mathrm{e}, 1}^{(i)}$, we find

$$
\begin{gathered}
c_{\mathrm{e}, 1}^{(2 k+1)}=0, \quad c_{\mathrm{e}, 1}^{(2 k+2)}=0, \\
b_{1}^{(2 k+1)}=\mathcal{C}_{k}^{(\mathrm{e}, 2)}\left\{-(2 k+1)\left(\frac{N}{2}+k\right) !+\frac{2}{\sqrt{\pi}}\left(\frac{N+1}{2}+k\right) !_{3} F_{2}\left[\begin{array}{c}
-k,-\frac{1}{2}-k,-\frac{1}{2} \\
\frac{1}{2},-\frac{1}{2}-k-\frac{N}{2} ; 1
\end{array}\right]\right\},
\end{gathered}
$$

and

$$
b_{1}^{(2 k+2)}=\mathrm{C}_{k}^{(\mathrm{e}, 4)}\left\{\frac{-1}{2 k+1}\left(\frac{N}{2}+k\right) !+\frac{1}{\sqrt{\pi}}\left(\frac{N-1}{2}+k\right) !_{3} F_{2}\left[\begin{array}{c}
-k, \frac{1}{2}-k, \frac{1}{2} \\
\frac{3}{2}, \frac{1}{2}-k-\frac{N}{2}
\end{array} ; 1\right]\right\},
$$

for $k=1,2, \cdots,(N / 2)-1$. We note that the explicit $k$ dependences on the right-hand sides of Eq. (6.45b) and Eq. 6.45a) are related to those on the right-hand sides of Eq. (6.17b) and 
Eq. (6.17c) by letting $k \rightarrow k-(1 / 2)$ and $k \rightarrow k+(1 / 2)$, respectively. Observe that the coefficients 6.453) of the modified Bessel functions $K_{1}, K_{3}, \cdots, K_{N-1}$ are trivially independent of $\omega$, while the initial values (5.45b) and (6.45c) of the $\omega=0$ recursion relation are independent of $\omega$ in view of Eqs. 6.40a) and (6.40b), respectively.

\subsection{4. $\omega$ dependence of the recursion relation (6.30)}

Equipped with the solutions 6.43 and 6.45) for the $\omega$ dependence of $b_{1}^{(i)}$ and $c_{\mathrm{e}, 1}^{(i)}$ with $i=1,2, \cdots, N$, we can derive the $\omega$ dependence of the coefficients $b_{m}^{(i)}$ with $m=2,3, \cdots$ in the limit $1 \leq m \ll \omega^{-1}$ from Eq. (6.39). Insertion of Eq. (6.43b) into the prefactors (6.40) appearing in Eq. 6.39) when $k=0$ gives

$$
\begin{aligned}
& b_{m}^{(1)}=\mathcal{C}_{0}^{(e, 2)} \frac{\left(m-\frac{1}{2}+\frac{N}{2}\right) !}{\left(m-\frac{1}{2}\right) !}-\mathcal{C}_{0}^{(e, 2)} \frac{\left(m-1+\frac{N}{2}\right) !}{4(m-1) !}(4-N \omega \ln \omega), \\
& b_{m}^{(2)}=\mathcal{C}_{0}^{(e, 4)} \frac{\left(m-\frac{3}{2}+\frac{N}{2}\right) !}{\left(m-\frac{3}{2}\right) !}-\mathcal{C}_{0}^{(e, 4)} \frac{\left(m-1+\frac{N}{2}\right) !}{4(m-1) !}(4+N \omega \ln \omega),
\end{aligned}
$$

where we have disregarded terms of order $\omega^{2}$ and higher. Insertion of Eq. (6.45b) into the prefactor (6.40a) appearing in Eq. (6.39a) gives

$$
\begin{aligned}
b_{m}^{(2 k+1)}= & \mathcal{C}_{k}^{(\mathrm{e}, 2)} \frac{\left(m+k-\frac{1}{2}+\frac{N}{2}\right) !}{\left(m-\frac{1}{2}\right) !}{ }_{3} F_{2}\left[\begin{array}{c}
-k,-\frac{1}{2}-k, \frac{1}{2}-m \\
\frac{1}{2}, \frac{1}{2}-k-m-\frac{N}{2}
\end{array} ; 1\right] \\
& -\mathcal{C}_{k}^{(\mathrm{e}, 2)}(2 k+1) \frac{\left(m+k-1+\frac{N}{2}\right) !}{(m-1) !}{ }_{3} F_{2}\left[\begin{array}{c}
-k, \frac{1}{2}-k, 1-m \\
\frac{3}{2}, 1-k-m-\frac{N}{2}
\end{array} ; 1\right]
\end{aligned}
$$

with $k=1,2, \cdots,(N / 2)-1$. Insertion of Eq. (6.45d) into the prefactor (6.40b) appearing in Eq. (6.39b) gives

$$
\begin{aligned}
b_{m}^{(2 k+2)}= & \mathcal{C}_{k}^{(\mathrm{e}, 4)} \frac{\left(m+k-\frac{3}{2}+\frac{N}{2}\right) !}{\left(m-\frac{3}{2}\right) !}{ }_{3} F_{2}\left[\begin{array}{c}
-k, \frac{1}{2}-k, \frac{3}{2}-m \\
\frac{3}{2}, \frac{3}{2}-k-m-\frac{N}{2} ; 1
\end{array}\right] \\
& -\mathcal{C}_{k}^{(\mathrm{e}, 4)} \frac{1}{2 k+1} \frac{\left(m+k-1+\frac{N}{2}\right) !}{(m-1) !}{ }_{3} F_{2}\left[\begin{array}{c}
-k,-\frac{1}{2}-k, 1-m \\
\frac{1}{2}, 1-k-m-\frac{N}{2} ; 1
\end{array}\right]
\end{aligned}
$$

with $k=1,2, \cdots,(N / 2)-1$. We emphasize again that the explicit $k$ dependences on the right-hand sides of Eq. (6.46c) and Eq. (5.46d) are related to those on the right-hand sides of Eq. 6.18b) and Eq. (6.18d) by letting $k \rightarrow k-(1 / 2)$ and $k \rightarrow k+(1 / 2)$, respectively.

In conclusion, the $\omega$ dependence of the solution to the recursion relation $(6.30)$ follows from combining Eqs. (6.35), 6.36), (6.43), 6.45a) when $m \gg N$ together with Eq. 6.46) when $m \omega \ll 1$. This gives 


$$
\begin{aligned}
& b_{m}^{(1)}= \begin{cases}+4 \mathcal{C}_{0}^{(\mathrm{e}, 2)} \frac{N \sqrt{\omega}}{8-N(N-2) \omega \ln \omega} m^{\frac{N-1}{2}} K_{1}(2 \sqrt{m \omega}), & N \ll m, \\
\mathcal{C}_{0}^{(e, 2)}\left(\frac{\left(m-\frac{1}{2}+\frac{N}{2}\right) !}{\left(m-\frac{1}{2}\right) !}-\frac{\left(m-1+\frac{N}{2}\right) !}{4(m-1) !}(4-N \omega \ln \omega)\right), & m \ll \omega^{-1},\end{cases} \\
& b_{m}^{(2)}= \begin{cases}-4 \mathcal{C}_{0}^{(e, 4)} \frac{N \sqrt{\omega}}{8-N(N-2) \omega \ln \omega} m^{\frac{N-1}{2}} K_{1}(2 \sqrt{m \omega}), & N \ll m, \\
\mathcal{C}_{0}^{(e, 4)}\left(\frac{\left(m-\frac{3}{2}+\frac{N}{2}\right) !}{\left(m-\frac{3}{2}\right) !}-\frac{\left(m-1+\frac{N}{2}\right) !}{4(m-1) !}(4+N \omega \ln \omega)\right), & m \ll \omega^{-1},\end{cases} \\
& b_{m}^{(2 k+1)}= \begin{cases}0, & N \ll m, \\
6.460), & m \ll \omega^{-1},\end{cases} \\
& b_{m}^{(2 k+2)}= \begin{cases}0, & N \ll m, \\
6.460), & m \ll \omega^{-1},\end{cases}
\end{aligned}
$$

with $k=1,2, \cdots,(N / 2)-1$. Equation (6.47) should be compared to Eq. (5.28). Observe that Eqs. (6.460) and (6.46d) are independent of $\omega$.

\subsubsection{Leading energy dependence of the density of states}

We are ready to extract the leading behavior of the average density of states $\nu(\varepsilon)$, Eq. (3.25), for asymptotically small energies $\varepsilon$. The density of states in the long wire limit Eq. (3.24) is given by

$$
\nu(\varepsilon)=\lim _{\omega \rightarrow-i \varepsilon} \pi^{-1} \operatorname{Re}_{\mathrm{L}}\left\langle\varphi_{0}|(\bar{B}-B)| \varphi_{0}\right\rangle_{\mathrm{R}},
$$

with the left and right ground states (4.10a) and 4.10b), respectively. The normalizations (4.8) and (4.11), the biorthogonal relations 4.9), and the identities

$$
\begin{aligned}
& (\bar{B}-B)|0\rangle=N|0\rangle, \\
& (\bar{B}-B)|m\rangle_{\mathrm{R}}^{(2 n+1)}=(N-4 n-1)|m\rangle_{\mathrm{R}}^{(2 n+1)}+|m\rangle_{\mathrm{L}}^{(2 n+1)}, \\
& (\bar{B}-B)|m\rangle_{\mathrm{R}}^{(2 n+2)}=(N-4 n-3)|m\rangle_{\mathrm{R}}^{(2 n+2)}-|m\rangle_{\mathrm{L}}^{(2 n+2)},
\end{aligned}
$$

deliver the expectation value

$$
\begin{aligned}
\mathrm{L}_{\langle}\left\langle\varphi_{0}|(\bar{B}-B)| \varphi_{0}\right\rangle_{\mathrm{R}}= & N+\sum_{m=1}^{\infty} \sum_{n=0}^{N / 2-1} \mathcal{N}_{m, n}^{(1)}\left(a_{m}^{(2 n+1)}\right)^{2}-\sum_{m=1}^{\infty} \sum_{n=0}^{N / 2-1} \mathcal{N}_{m, n}^{(2)}\left(a_{m}^{(2 n+2)}\right)^{2} \\
= & N+\sum_{m=1}^{\infty} \sum_{n=0}^{N / 2-1} \mathcal{N}_{m, n}^{(1)}\left(\sum_{k=0}^{N / 2-1}\left[\mathcal{M}_{\mathrm{e} ; N, 1}^{-1}\right]_{k}^{n} b_{m}^{(2 k+1)}\right)^{2} \\
& -\sum_{m=1}^{\infty} \sum_{n=0}^{N / 2-1} \mathcal{N}_{m, n}^{(2)}\left(\sum_{k=0}^{N / 2-1}\left[\mathcal{M}_{\mathrm{e} ; N, 2}^{-1}\right]_{k}^{n} b_{m}^{(2 k+2)}\right)^{2}
\end{aligned}
$$

The matrices $\mathcal{M}_{\mathrm{e} ; N, 1}$ and $\mathcal{M}_{\mathrm{e} ; N, 2}$ and their inverses are given in Eq. 6.29) while the normalization factors $\mathcal{N}_{m, n}^{(1)}$ and $\mathcal{N}_{m, n}^{(2)}$ are given by Eq. (4.8). In order to evaluate Eq. (6.50) it is necessary to break the sum over $m$ into two parts separated by the integer $1 \ll m_{0} \sim \omega^{-1}$. This gives

$$
{ }_{\mathrm{L}}\left\langle\varphi_{0}|(\bar{B}-B)| \varphi_{0}\right\rangle_{\mathrm{R}}=N+S_{1}+S_{2}
$$


with

$$
\begin{aligned}
& S_{1}:=\sum_{m=1}^{m_{0}} \sum_{n=0}^{N / 2-1} \mathcal{N}_{m, n}^{(1)}\left(a_{m}^{(2 n+1)}\right)^{2}-\sum_{m=1}^{m_{0}} \sum_{n=0}^{N / 2-1} \mathcal{N}_{m, n}^{(2)}\left(a_{m}^{(2 n+2)}\right)^{2}, \\
& S_{2}:=\sum_{m=m_{0}+1}^{\infty} \sum_{n=0}^{N / 2-1}\left[\mathcal{N}_{m, n}^{(1)}\left(\left[\mathcal{M}_{\mathrm{e} ; N, 1}^{-1}\right]_{0}^{n} b_{m}^{(1)}\right)^{2}-\mathcal{N}_{m, n}^{(2)}\left(\left[\mathcal{M}_{\mathrm{e} ; N, 2}^{-1}\right]_{0}^{n} b_{m}^{(2)}\right)^{2}\right] .
\end{aligned}
$$

Here, we made use of the fact that in the large $m$ limit the coefficients $b_{m}^{(2 k+1)}$ and $b_{m}^{(2 k+2)}$ all vanish except for $b_{m}^{(1)}$ and $b_{m}^{(2)}$. We are going to compute the sum $S_{1}$ for $m \leq m_{0}$ and the sum $S_{2}$ for $m>m_{0}$ separately.

To compute the sum $m \leq m_{0}, S_{1}$, we first need to derive the leading $\omega$ dependence of the coefficients $a_{m}^{(2 n+1)}$ and $a_{m}^{(2 n+2)}$. Inserting Eq. (6.46a) and (6.46c) into Eq. (6.29c) yields

$$
a_{m}^{(2 n+1)}=+\frac{(-1)^{n} \sqrt{2}\left(\frac{N}{2}+m-1\right) !}{(m+n)(m-1) !\left(\frac{N}{2}-n-1\right) ! n !}[1+(m+n) \omega \ln \omega],
$$

where $n=0,1, \cdots,(N / 2)-1$. Inserting Eq. (6.46b) and (6.46d) into Eq. (6.29d) gives

$$
a_{m}^{(2 n+2)}=-\frac{(-1)^{n} \sqrt{2}\left(\frac{N}{2}+m-1\right) !}{(m+n)(m-1) !\left(\frac{N}{2}-n-1\right) ! n !}[1+(m+n) \omega \ln \omega],
$$

where $n=0,1, \cdots,(N / 2)-1$. With this, the normalization factors (4.8), and the choice $m_{0}=$ $\omega^{-1}$, we find

$$
\begin{aligned}
S_{1}= & \sum_{m=1}^{1 / \omega} \sum_{n=0}^{N / 2-1}\left(\mathcal{N}_{m, n}^{(1)}\left(a_{m}^{(2 n+1)}\right)^{2}-\mathcal{N}_{m, n}^{(2)}\left(a_{m}^{(2 n+2)}\right)^{2}\right) \\
\approx & \frac{2}{\pi} \sum_{m=1}^{1 / \omega} \frac{\left(\frac{N}{2}+m-1\right) !}{(m-1) !} \sum_{n=0}^{N / 2-1} \frac{(1+(m+n) \omega \ln \omega)^{2}}{(m+n)\left(\frac{N}{2}-n-1\right) ! n !} \\
& \times\left(\frac{\left(m-\frac{1}{2}\right) !\left(\frac{N}{2}-n-\frac{1}{2}\right) !\left(n-\frac{1}{2}\right) !}{\left(\frac{N}{2}+m-\frac{1}{2}\right) !}-\frac{\left(m-\frac{3}{2}\right) !\left(\frac{N}{2}-n-\frac{3}{2}\right) !\left(n+\frac{1}{2}\right) !}{\left(\frac{N}{2}+m-\frac{3}{2}\right) !}\right),
\end{aligned}
$$

which is to be compared with the telescopic expansion (5.32). To compute the leading behavior of the density of states it is sufficient to retain only the lowest order in $\omega$. This gives

$$
S_{1} \approx-N-\text { const } \times \omega \ln ^{2} \omega,
$$

to leading and subleading orders in $\omega$.

For the sum over $m>m_{0}, S_{2}$, we obtain by inserting the definition of $\mathcal{M}_{\mathrm{e} ; N, 1}^{-1}, \mathcal{M}_{\mathrm{e} ; N, 2}^{-1}, \mathcal{N}_{m, n}^{(1)}$, and $\mathcal{N}_{m, n}^{(2)}$, as well as the solutions for $b_{m}^{(1)}$ and $b_{m}^{(2)}$ from Eq. (6.47)

$$
S_{2}=\sum_{m=m_{0}+1}^{\infty} \mathcal{S}_{N}^{(\mathrm{e})}(m) \frac{(4 N)^{2} \omega m^{N-1} K_{1}^{2}(2 \sqrt{m \omega})}{[8-N(N-2) \omega \ln \omega]^{2}}
$$

with the combinatorial factor

$$
\mathcal{S}_{N}^{(\mathrm{e})}(m):=\sum_{n=0}^{N / 2-1}\left[\mathcal{N}_{m, n}^{(1)}\left(\mathcal{C}_{0}^{(e, 2)}\left[\mathcal{M}_{e, N, 1}^{-1}\right]_{0}^{n}\right)^{2}-\mathcal{N}_{m, n}^{(2)}\left(\mathcal{C}_{0}^{(e, 4)}\left[\mathcal{M}_{e, N, 2}^{-1}\right]_{0}^{n}\right)^{2}\right]
$$


After converting the sum in $S_{2}$ into an integral, we find

$$
S_{2}=\int_{m_{0}+1}^{\infty} d m \mathcal{S}_{N}^{(\mathrm{e})}(m) \frac{(4 N)^{2} \omega m^{N-1} K_{1}^{2}(2 \sqrt{m \omega})}{[8-N(N-2) \omega \ln \omega]^{2}} .
$$

Using the substitution $x^{2}=4 m \omega$ the integral transforms into

$$
S_{2}=\frac{(4 N)^{2} \omega^{1-N}}{[8-N(N-2) \omega \ln \omega]^{2}} \int_{x_{0}}^{\infty} d x \mathcal{S}_{N}^{(\mathrm{e})}\left(\frac{x^{2}}{4 \omega}\right)\left(\frac{x}{2}\right)^{2 N-1} K_{1}^{2}(x),
$$

with $x_{0}=2 \sqrt{\left(m_{0}+1\right) \omega} \sim \mathcal{O}(1)$, since we chose $m_{0}=\omega^{-1}$. Let us now expand $\mathcal{S}_{N}^{(\mathrm{e})}$ in small $\omega$ (i.e., large argument). This gives

$$
\mathcal{S}_{N}^{(\mathrm{e})}\left(\frac{x^{2}}{4 \omega}\right) \approx \mathcal{S}_{N}^{(\mathrm{e}, 1)} \times\left(\frac{2 \omega}{x^{2}}\right)^{N-1}+\mathcal{O}\left[\left(\frac{2 \omega}{x^{2}}\right)^{N}\right],
$$

where the prefactor to the $\omega^{N-1}$ term,

$$
\begin{aligned}
\mathcal{S}_{N}^{(\mathrm{e}, 1)}:=\frac{8}{\left(\frac{N}{2}\right) !^{2}} \sum_{n=0}^{N / 2-1}\{ & \frac{[(N-1) !]^{2}\left({ }_{2} F_{1}\left[-\frac{N}{2},-n, \frac{1}{2}-\frac{N}{2}, 1\right]\right)^{2}}{(N-2 n-1) !(2 n) !} \\
& \left.-\frac{[(N-2) !]^{2}\left({ }_{2} F_{1}\left[-\frac{N}{2},-n, \frac{3}{2}-\frac{N}{2}, 1\right]\right)^{2}}{(N-2 n-2) !(2 n+1) !}\right\} \\
=0 &
\end{aligned}
$$

is in fact vanishing. Here, we have introduced the Gauss hypergeometric function ${ }_{2} F_{1}$. Hence, we conclude that the leading term of $S_{2}$ is (at most) of order $\omega$, and can therefore be neglected compared to $S_{1}$, whose subleading $\omega$ dependence is of order $\omega \ln \omega$. The above reasoning also justifies the neglect of the $m>m_{0}$ sum in Eq. (5.31).

Combining Eq. (6.51) and (6.55), we find that the density of states, for asymptotically small energies, is given by

$$
\begin{aligned}
\nu(\varepsilon) & =\lim _{\omega \rightarrow-i \varepsilon} \pi^{-1} \operatorname{Re}\left(N+S_{1}+S_{2}\right) \\
& \propto \lim _{\omega \rightarrow-i \varepsilon} \pi^{-1} \operatorname{Re}\left(-\omega \ln ^{2} \omega\right) \\
& \propto \varepsilon|\ln \varepsilon| .
\end{aligned}
$$

Recalling that $\varepsilon$ is measured in units of the disorder strength, we have recovered Eq. (1.1) when the number of channels $N$ is even.

\section{Discussion}

The asymptotic limit $0<\varepsilon \ll 1$ for the density of states 1.1. of a disordered quasi-onedimensional quantum wire in the chiral-unitary symmetry class was first derived in Refs. [82] and [83] using the DMPK approach. This approach is based on finding the stationary solution of a forced diffusive process obeyed by the Lyapunov exponents of a transfer matrix, whereby the length $L$ of the quasi-one-dimensional quantum wire plays the role of time and the forcing term is proportional to the frequency $\omega$ related to the energy $\varepsilon$ by the analytical continuation 
$\omega \rightarrow-i \varepsilon$. The DMPK approach is geometric, for the Lyapunov exponents are none but the radial coordinates of a smooth manifold determined by the symmetry class to which the disorder belongs. This symmetry requirement, the quasi-one-dimensionality, and the implicit weakness of the disorder determines in a unique way the forced diffusive process in the thick quantum wire limit $N \rightarrow \infty$ [82 87]. In this paper, we have derived the density of states (1.1) using the alternative superspin approach.

The superspin approach relies on interpreting the disorder-average retarded Green function as a "quantum thermal average", whereby the role of temperature is played by the length $L$ of the disordered quantum wire and, for a disordered quasi-one-dimensional quantum wire, the quantum partition function is given by Eqs. (3.8) and (3.10). The quantum evolution, although unitary according to Eq. 3.22), is here generated by the supersymmetric and non-Hermitian Hamiltonian (3.8). In the chiral-unitary symmetry class, the supersymmetric and non-Hermitian Hamiltonian (3.8) simplifies to the supersymmetric and non-Hermitian Hamiltonian (3.12) so that it can be interpreted as a $g l(1 \mid 1)$ quantum superspin Hamiltonian, for it reduces to a bilinear form in the generators of the superalgebra $g l(2 \mid 2)$ and, furthermore, it commutes with a $g l(1 \mid 1)$ sub-superalgebra of $g l(2 \mid 2)$. In the thermodynamic limit $L \rightarrow \infty$, the quantum statistical average is solely determined by the non-degenerate right and left ground states of Hamiltonian (3.12), whose existence is guaranteed by supersymmetry. Thus, the computation of the density of states in the thermodynamic limit amounts to the construction of the right and left ground states (4.10) for the supersymmetric and non-Hermitian Hamiltonian (3.12).

Solving for these right and left ground states is difficult because the right and left Hilbert spaces on which the quantum superspin Hamiltonian is defined are infinite dimensional. For comparison, the irreducible Hilbert space of a single $S U(2)$-spin is $(2 s+1)$-dimensional. For any given number $N$ of channels in the disordered quasi-one-dimensional quantum wire, the right and left ground states are constructed from two limiting solutions to the recursion relation (4.13) for their expansion coefficients $a_{m}^{(n)}$. Here, $n=1, \cdots, N$ is the channel index and $m=1,2, \cdots$ is the basis index in the right Hilbert space. More precisely, the recursion relation 4.13) was solved independently in the large $m \gg N$ and in the small $m \omega \ll 1$ limits, respectively, with the help of a change of basis in the right Hilbert space, i.e., by trading the expansion coefficients $a_{m}^{(n)}$ for the expansion coefficients $b_{m}^{(k)}, k=1, \cdots, N$. In the large $m \gg N$ limit, the recursion relation (4.13) reduces to $N$ modified Bessel equations whose leading solution for small $\omega$ is governed by (i) the modified Bessel function $K_{0}$ when $N$ is odd or (ii) the modified Bessel function $K_{1}$ when $N$ is even. Evidently, this parity effect also characterizes the solution to the recursion relation in the limit $m \omega \ll 1$ provided $N \ll m \ll 1 / \omega$, after both solutions have been matched in their overlapping regime $N \ll m \ll 1 / \omega$ of validity. In turn, the same parity effect has a counterpart for the density of states for asymptotically small values of $\varepsilon$, since, upon the analytical continuation $\omega \rightarrow-\mathrm{i} \varepsilon$, (i) the coefficients $b_{m}^{(1)}$ dominate over the coefficients $b_{m}^{(k)}$ with $k=2, \cdots, N$ in the density of states when $N$ is odd, while (ii) the coefficients $b_{m}^{(1)}$ and $b_{m}^{(2)}$ dominate over the coefficients $b_{m}^{(k)}$ with $k=3, \cdots, N$ in the density of states when $N$ is even.

In the superspin approach, the expansion coefficients $b_{m}^{(1)}$ when $N$ is odd and $b_{m}^{(1)}$ and $b_{m}^{(2)}$ when $N$ is even play the role, in the DMPK approach, of the smallest Lyapunov exponent when $N$ is odd and the first two smallest Lyapunov exponents when $N$ is even, respectively. The only effect from the number $N$ of channels is to determine the size of the asymptotic regime $\varepsilon \ll 1$ for which Eq. 1.1) holds through the choice $g_{2} \propto v_{\mathrm{F}} /\left(N^{2} \ell\right)$, where $v_{\mathrm{F}}$ is the Fermi velocity in the clean limit and $\ell$ is the mean free path in the Born approximation, for the variance in Eq. (2.7). 


\section{Acknowledgments}

We thank A. W. W. Ludwig for helpful discussions. This research was supported in part by the National Science Foundation under Grant No. PHY05-51164.

\section{Appendix A. Symmetries of the Hamiltonian}

In this appendix we will show that $H_{2}$ [as defined in Eq. (3.12)] commutes with the eight generators of the Lie superalgebra $g l(1 \mid 1) \oplus g l(1 \mid 1)$, while $H_{\omega}$ commutes with the four generators of the diagonal sub-algebra $g l(1 \mid 1) \subset g l(1 \mid 1) \oplus g l(1 \mid 1)$. It thus follows that $H$ is invariant under all transformations of $\left(\mathfrak{F}_{L}, \mathfrak{F}_{R}\right)$ induced by the Lie supergroup $G L(1 \mid 1)$ generated by the Lie superalgebra $g l(1 \mid 1)$. We refer to Ref. [95] for an introduction on supermanifolds, to Ref. [96] for an introduction on Lie superalgebra, and to Ref. [94] for a dictionary of Lie superalgebras.

The first step in the proof consists in rewriting $\mathrm{H}_{2}$ as a quadratic form in the generators of the Lie superalgebra $g l(2 \mid 2)$. To this end, define the 16 operators $E_{a b}$ with $a, b=1, \cdots, 4$ by: bosonic operators in the nonbar sector

$$
E_{11} \equiv-B:=-\sum_{i=1}^{N} f_{i}^{\dagger} f_{i}+\frac{1}{2} N, \quad E_{33} \equiv-Q:=-\sum_{i=1}^{N} b_{i}^{\dagger} b_{i}-\frac{1}{2} N,
$$

fermionic operators in the nonbar sector

$$
E_{13} \equiv+W_{+}:=+\sum_{i=1}^{N} b_{i}^{\dagger} f_{i}, \quad E_{31} \equiv-W_{-}:=-\sum_{i=1}^{N} f_{i}^{\dagger} b_{i},
$$

bosonic operators in the bar sector

$$
E_{22} \equiv-\bar{B}:=-\sum_{i=1}^{N} \bar{f}_{i} \bar{f}_{i}^{\dagger}+\frac{1}{2} N, \quad E_{44} \equiv-\bar{Q}:=+\sum_{i=1}^{N} \bar{b}_{i} \bar{b}_{i}^{\dagger}-\frac{1}{2} N,
$$

fermionic operators in the bar sector

$$
E_{24} \equiv+\bar{W}_{+}:=-\sum_{i=1}^{N} \bar{b}_{i} \bar{f}_{i}^{\dagger}, \quad \quad E_{42} \equiv-\bar{W}_{-}:=-\sum_{i=1}^{N} \bar{f}_{i} \bar{b}_{i}^{\dagger},
$$

bosonic operators in the mixed bar and nonbar sector

$$
\begin{array}{ll}
E_{12} \equiv-A_{+}:=-\sum_{i=1}^{N} f_{i} \bar{f}_{i}, & E_{21} \equiv+A_{-}:=+\sum_{i=1}^{N} f_{i}^{\dagger} \bar{f}_{i}^{\dagger}, \\
E_{34} \equiv+D_{+}:=-\sum_{i=1}^{N} b_{i} \bar{b}_{i}, & E_{43} \equiv+D_{-}:=+\sum_{i=1}^{N} b_{i}^{\dagger} \bar{b}_{i}^{\dagger},
\end{array}
$$

fermionic operators in the mixed bar and nonbar sector

$$
\begin{array}{ll}
E_{14} \equiv-S_{+}:=+\sum_{i=1}^{N} f_{i} \bar{b}_{i}, & E_{41} \equiv+S_{-}:=+\sum_{i=1}^{N} f_{i}^{\dagger} \bar{b}_{i}^{\dagger}, \\
E_{23} \equiv-\bar{S}_{+}:=-\sum_{i=1}^{N} b_{i}^{\dagger} \bar{f}_{i}^{\dagger}, & E_{32} \equiv+\bar{S}_{-}:=+\sum_{i=1}^{N} b_{i} \bar{f}_{i} .
\end{array}
$$

The 16 operators $E_{a b}$ with $a, b=1, \cdots, 4$ are either bosonic or fermionic. Hence, any $E_{a b}$ with $a, b=1, \cdots, 4$ can be assigned the degree (or grade) 0 or 1 if they are bosonic or fermionic, 
respectively, a mapping that we shall denote by $\operatorname{deg}\left(E_{a b}\right)$. Our rational for the definition of the 16 operators $E_{a b}$ with $a, b=1, \cdots, 4$ is that if we define

$$
[a]:= \begin{cases}0, & \text { if } a=1,2, \\ 1, & \text { if } a=3,4,\end{cases}
$$

then it follows that

$$
\operatorname{deg}\left(E_{a b}\right)=([a]+[b]) \bmod 2 .
$$

We can now define the 256 supercommutators

$$
\left[E_{a b}, E_{c d}\right]:=E_{a b} E_{c d}-(-1)^{\operatorname{deg}\left(E_{a b}\right) \operatorname{deg}\left(E_{c d}\right)} E_{c d} E_{a b}, \quad a, b, c, d=1, \cdots, 4,
$$

and verify that they define the Lie superalgebra of $g l(2 \mid 2)$ in the standard basis [97], i.e.,

$$
\left[E_{a b}, E_{c d}\right]=\delta_{b c} E_{a d}-(-1)^{([a]+[b])([c]+[d])} \delta_{a d} E_{c b}, \quad a, b, c, d=1, \cdots, 4 .
$$

The first step of the proof is then completed after verifying that $H_{2}$, as given by Eqs. (3.12) and (3.8c), can be rewritten as

$$
\begin{aligned}
H_{2}=g_{2}[ & \left(A_{+}\right)^{2}+2 B \bar{B}-2 S_{-} \bar{S}_{+}-2 W_{-} \bar{W}_{+}+\left(A_{-}\right)^{2} \\
& \left.+2 \bar{W}_{-} W_{+}+2 \bar{S}_{-} S_{+}-\left(D_{+}\right)^{2}-2 Q \bar{Q}-\left(D_{-}\right)^{2}\right],
\end{aligned}
$$

or alternatively

$$
\begin{aligned}
H_{2}=g_{2}[ & \left(E_{12}\right)^{2}+2 E_{11} E_{22}+2 E_{41} E_{23}+2 E_{31} E_{24}+\left(E_{21}\right)^{2} \\
& \left.+2 E_{42} E_{13}+2 E_{32} E_{14}-\left(E_{34}\right)^{2}-2 E_{33} E_{44}-\left(E_{43}\right)^{2}\right] .
\end{aligned}
$$

Observe that

$$
H_{\omega}=\omega(B-\bar{B}+Q-\bar{Q})=\omega\left(E_{22}-E_{11}+E_{44}-E_{33}\right) .
$$

In the second step, one verifies that $H_{2}$ commutes with the 8 operators

$$
\begin{array}{lll}
\mathcal{G}_{1}:=\frac{1}{2}\left(-E_{33}-E_{44}+E_{34}+E_{43}\right), & \mathcal{H}_{1}:=\frac{1}{2}\left(-E_{11}-E_{22}+E_{12}+E_{21}\right), \\
\mathcal{E}_{1}^{+}:=\frac{1}{2}\left(+E_{13}+E_{24}-E_{14}-E_{23}\right), & \mathcal{E}_{1}^{-}:=\frac{1}{2}\left(-E_{31}-E_{42}+E_{41}+E_{32}\right), \\
\mathcal{G}_{2}:=\frac{1}{2}\left(-E_{33}-E_{44}-E_{34}-E_{43}\right), & \mathcal{H}_{2}:=\frac{1}{2}\left(-E_{11}-E_{22}-E_{12}-E_{21}\right), \\
\mathcal{E}_{2}^{+}:=\frac{1}{2}\left(+E_{13}+E_{24}+E_{14}+E_{23}\right), & \mathcal{E}_{2}^{-}:=\frac{1}{2}\left(-E_{31}-E_{42}-E_{41}-E_{32}\right),
\end{array}
$$

that generate the Lie superalgebra $g l(1 \mid 1) \oplus g l(1 \mid 1)$ with the only nonvanishing supercommutators [98,99]

$$
\begin{aligned}
& {\left[\mathcal{G}_{1}, \mathcal{E}_{1}^{ \pm}\right]= \pm \mathcal{E}_{1}^{ \pm}, \quad\left[\mathcal{H}_{1}, \mathcal{E}_{1}^{ \pm}\right]=\mp \mathcal{E}_{1}^{ \pm}, \quad\left\{\mathcal{E}_{1}^{+}, \mathcal{E}_{1}^{-}\right\}=\mathcal{G}_{1}+\mathcal{H}_{1},} \\
& {\left[\mathcal{G}_{2}, \mathcal{E}_{2}^{ \pm}\right]= \pm \mathcal{E}_{2}^{ \pm}, \quad\left[\mathcal{H}_{2}, \mathcal{E}_{2}^{ \pm}\right]=\mp \mathcal{E}_{2}^{ \pm}, \quad\left\{\mathcal{E}_{2}^{+}, \mathcal{E}_{2}^{-}\right\}=\mathcal{G}_{2}+\mathcal{H}_{2} .}
\end{aligned}
$$


At last, one verifies that the full Hamiltonian $H=H_{\omega}+H_{2}$ commutes with the 4 operators

$$
\begin{aligned}
& \mathcal{B}:=\mathcal{H}_{1}+\mathcal{H}_{2}=\sum_{i=1}^{N}\left(f_{i}^{\dagger} f_{i}-\bar{f}_{i}^{\dagger} \bar{f}_{i}\right), \\
& \mathcal{Q}:=\mathcal{G}_{1}+\mathcal{G}_{2}=\sum_{i=1}^{N}\left(b_{i}^{\dagger} b_{i}+\bar{b}_{i}^{\dagger} \bar{b}_{i}\right), \\
& \mathcal{W}_{+}:=\mathcal{E}_{1}^{+}+\mathcal{E}_{2}^{+}=\sum_{i=1}^{N}\left(b_{i}^{\dagger} f_{i}+\bar{f}_{i}^{\dagger} \bar{b}_{i}\right), \\
& \mathcal{W}_{-}:=\mathcal{E}_{1}^{-}+\mathcal{E}_{2}^{-}=\sum_{i=1}^{N}\left(f_{i}^{\dagger} b_{i}+\bar{b}_{i}^{\dagger} \bar{f}_{i}\right),
\end{aligned}
$$

that generate the Lie superalgebra $g l(1 \mid 1)$ whose only nonvanishing supercommutators are

$$
\left[\mathcal{Q}-\mathcal{B}, \mathcal{W}_{ \pm}\right]= \pm 2 \mathcal{W}_{ \pm}, \quad\left\{\mathcal{W}_{+}, \mathcal{W}_{-}\right\}=\mathcal{Q}+\mathcal{B}
$$

Thus, $H_{\omega}$ breaks the symmetry of $H_{2}$ generated by $g l(1 \mid 1) \oplus g l(1 \mid 1)$ down to the one generated by $g l(1 \mid 1)$.

\section{Appendix B. Useful identities}

The generalized hypergeometric functions have the series expansion 93

$$
{ }_{p} F_{q}\left[\begin{array}{c}
\alpha_{1}, \cdots, \alpha_{p} \\
\beta_{1}, \cdots, \beta_{p}
\end{array} ; z\right]=\sum_{n=0}^{\infty} \frac{\left(\alpha_{1}\right)_{n} \cdots\left(\alpha_{p}\right)_{n}}{\left(\beta_{1}\right)_{n} \cdots\left(\beta_{q}\right)_{n}} \frac{z^{n}}{n !}
$$

where $\alpha_{1}, \cdots, \alpha_{p}$ and $\beta_{1}, \cdots, \beta_{q}$ are complex-valued parameters, $z$ is complex-valued with magnitude less than unity, and

$$
(\alpha)_{n}:=\frac{\Gamma(\alpha+n)}{\Gamma(\alpha)}
$$

with $\Gamma(z)$ the gamma function, denotes the Pochhammer symbol.

We introduce the short-hand notations

$$
\begin{aligned}
& F_{1}[k, m, n]:={ }_{3} F_{2}\left[\begin{array}{c}
-k, k,-n \\
\frac{1}{2},-m,
\end{array}\right] 1, \\
& F_{2}[k, m, n]:={ }_{3} F_{2}\left[\begin{array}{c}
-k, 2+k,-n \\
\frac{3}{2},-m,
\end{array}\right], \\
& F_{3}[k, m, n]:={ }_{3} F_{2}\left[\begin{array}{c}
-k, k+1,-n \\
\frac{1}{2},-m,
\end{array}\right], \\
& F_{4}[k, m, n]:={ }_{3} F_{2}\left[\begin{array}{c}
-k, k+1,-n \\
\frac{3}{2},-m,
\end{array}\right],
\end{aligned}
$$


for the family of hypergeometric functions labeled by the positive integers $k, m$, and $n$. They satisfy the identities

$$
\begin{aligned}
0= & {\left[4 n(2 n-2 m)-\left(2 m-4 k^{2}\right)\right] F_{1}[k, m, n] } \\
& +2(2 n+1)(m-n) F_{1}[k, m, n+1]+2 n(2 m+1-2 n) F_{1}[k, m, n-1], \\
0= & \left\{4 n(2 n-2 m)-\left[6 m+4-4(k+1)^{2}\right]\right\} F_{2}[k, m, n] \\
& +2(2 n+3)(m-n) F_{2}[k, m, n+1]+2 n(2 m+3-2 n) F_{2}[k, m, n-1], \\
0= & \{4 n(2 n-2 m-1)-[2 m-4 k(k+1)]\} F_{3}[k, m, n] \\
& +2(2 n+1)(m-n) F_{3}[k, m, n+1]+2 n(2 m+3-2 n) F_{3}[k, m, n-1], \\
0= & \{4 n(2 n+1-2 m)-[6 m-4 k(k+1)]\} F_{4}[k, m, n] \\
& +(2 n+3)(2 m-2 n) F_{4}[k, m, n+1]+2 n(2 m+1-2 n) F_{4}[k, m, n-1],
\end{aligned}
$$

respectively.

Furthermore, we introduce the notation

$$
\begin{aligned}
& F_{3}^{-1}[k, m, n]:=\frac{1}{(m-k) !(m+k+1) !}{ }^{3} F_{2}\left[\begin{array}{c}
-k-m-1, k-m,-n \\
-m-\frac{1}{2},-m,
\end{array} ; 1,\right. \\
& F_{4}^{-1}[k, m, n]:=\frac{(2 k+1)^{2}}{(m-k) !(m+k+1) !}{ }^{3} F_{2}\left[\begin{array}{c}
-k-m-1, k-m,-n \\
-m, \frac{1}{2}-m,
\end{array}\right] \text {, }
\end{aligned}
$$

with the positive integers $k, m$, and $n$. They satisfy the identities

$$
\begin{aligned}
& \sum_{k=0}^{m} F_{3}^{-1}[k, m, n] F_{3}\left[k, m, n^{\prime}\right]=(-1)^{n} 4^{m} \frac{n !(m-n) !}{m !(2 m+1) !} \delta_{n, n^{\prime}}, \\
& \sum_{k=0}^{m} F_{4}^{-1}[k, m, n] F_{4}\left[k, m, n^{\prime}\right]=(-1)^{n} 4^{m} \frac{n !(m-n) !}{m !(2 m) !} \delta_{n, n^{\prime}},
\end{aligned}
$$

for $n, n^{\prime} \in\{0,1,2, \cdots, m\}$.

\section{References}

[1] P. W. Anderson, Phys. Rev. 109, 1492 (1958).

[2] P. A. Lee and R. V. Ramakrishnan, Rev. Mod. Phys. 57, 287 (1985).

[3] B. Kramer and A. MacKinnon, Rep. Prog. Phys. 56, 1469 (1993).

[4] B. Kramer, T. Ohtsuki, and S. Kettemann, Phys. Rep. 417, 211 (2005).

[5] F. Evers and A. D. Mirlin, Rev. Mod. Phys. 80, 1355 (2008).

[6] J. Fröhlich and T. Spencer, Commun. Math. Phys. 88, 151 (1983).

[7] F. J. Dyson, J. Math. Phys. 6, 1199 (1962).

[8] J. J. M. Verbaarschot, Phys. Rev. Lett. 72, 2531 (1994).

[9] M. R. Zirnbauer, J. Math. Phys. 37, 4986 (1996).

[10] A. Altland and M. R. Zirnbauer, Phys. Rev. B 55, 1142 (1997).

[11] M. Caselle and W. Magnea, Phys. Rep. 394, 41 (2004).

[12] P. Heinzner, A. Huckleberry, and M. R. Zirnbauer, Commun. Math. Phys. 257, 725 (2005). 
[13] S. Helgason, Differential Geometry, Lie Groups, and Symmetric Spaces (Academic Press, San Diego, 1978).

[14] Slightly abusing terminology, we also call a quantum critical point that separates two insulating phases (instead of separating a metallic from an insulating phase) a mobility edge in a problem of Anderson localization.

[15] F. J. Wegner, Z. Physik B 35, 207 (1979).

[16] S. Hikami, A. I. Larkin, and Y. Nagaoka, Prog. Theor. Phys. 63, 707 (1980).

[17] L. Schäfer and R. J. Wegner, Z. Physik B 38, 113 (1980).

[18] K. B. Efetov, A. I. Larkin, and D. E. Khemlnitskii, Zh. Eksp. Teor. Fiz. 79, 1120 (1980) [Sov. Phys. JETP 52, 568 (1980)].

[19] A. Houghton, A. Jevicky, R. D. Kenway, and A. M. M. Pruisken, Phys. Rev. Lett. 45, 394 (1980).

[20] S. Hikami, Phys. Rev. B 24, 2671 (1981).

[21] K. B. Efetov, Adv. Phys. 32, 53 (1983).

[22] H. Levine, S. B. Libby, and A. M. M. Pruisken, Phys. Rev. Lett. 51, 1915 (1983); A. M. M. Pruisken, Nucl. Phys. B 235, 277 (1984).

[23] R. Oppermann, Physica (Amsterdam) 167A, 301 (1990).

[24] R. Gade, Nucl. Phys. B 398, 499 (1993); R. Gade and F. Wegner, ibid. 360, 213 (1991).

[25] M. R. Zirnbauer, Phys. Rev. Lett. 69, 1584 (1992).

[26] R. Bundschuh, C. Cassanello, D. Serban, and M. R. Zirnbauer, Nucl. Phys. B 532, 689 (1998); ibid Phys. Rev. B 59, 4382 (1999).

[27] T. Senthil, M. P. A. Fisher, L. Balents, and C. Nayak, Phys. Rev. Lett. 81, 4704 (1998); T. Senthil and M. P. A. fisher, Phys. Rev. B 60, 6893 (1999).

[28] T. Senthil and M. P. A. Fisher, Phys. Rev. B 61, 9690 (2000).

[29] M. Bocquet, D. Serban, and M. R. Zirnbauer, Nucl. Phys. B 578, 628 (2000).

[30] N. Read and D. Green, Phys. Rev. B 61, 10267 (2000).

[31] S. Guruswamy, A. LeClair, and A. W. W. Ludwig, Nucl. Phys. B 583, 475 (2000).

[32] P. Fendley, Phys. Rev. B 63, 104429 (2001).

[33] A. Altland and R. Merkt, Nucl. Phys. B 607, 511 (2001).

[34] A. Lamacraft, B. D. Simons, and M. R. Zirnbauer, Phys. Rev. B 70, 075412 (2004).

[35] S. Ryu, A. Furusaki, A. W. W. Ludwig, and C. Mudry, Nucl. Phys. B 780, 105 (2007).

[36] P. M. Ostrovsky, I. V. Gornyi, and A. D. Mirlin, Phys. Rev. Lett. 98, 256801 (2007).

[37] S. Ryu, C. Mudry, H. Obuse, and A. Furusaki, Phys. Rev. Lett. 99, 116601 (2007).

[38] J. T. Chalker and P. D. Coddington, J. Phys. C 21, 2665 (1988).

[39] D. K. K. Lee and J. T. Chalker, Phys. Rev. Lett. 72, 1510 (1994); D. K. K. Lee, J. T. Chalker, and D. Y. K. Ko, Phys. Rev. B 50, 5272 (1994).

[40] Y. B. Kim, A. Furusaki, and D. K. Lee, Phys. Rev. B 52, 16646 (1995).

[41] J. T. Chalker and A. Dohmen, Phys. Rev. Lett. 75, 4496 (1995).

[42] R. Klesse and R. Metzler, Europhys. Lett. 32, 229 (1995).

[43] C.-M. Ho and J.T. Chalker, Phys. Rev. B 54, 8708 (1996).

[44] S. Cho and M. P. A. Fisher, Phys. Rev. B 55, 1025 (1997).

[45] V. Kagalovsky, B. Horovitz, and Y. Avishai, Phys. Rev. B 55, 7761 (1997).

[46] R. Merkt, M. Janssen, and B. Huckestein, Phys. Rev. B 58, 4394 (1998).

[47] V. Kagalovsky, B. Horovitz, Y. Avishai, and J.T. Chalker, Phys. Rev. Lett. 82, 3516 (1999).

[48] J. T. Chalker, N. Read, V. Kagalovsky, B. Horovitz, Y. Avishai, and A. W. W. Ludwig, Phys. Rev. B 65, 012506 (2001).

[49] M. Bocquet and J. T. Chalker, Phys. Rev. B 67, 054204 (2003).

[50] H. Obuse, A. Furusaki, S. Ryu, C. Mudry, Phys. Rev. B 76, 075301 (2007); ibid, Phys. Rev. B 78, 115301 (2008).

[51] B. Huckestein, Rev. Mod. Phys. 67, 357 (1995).

[52] N. Read, unpublished, 1991.

[53] D.-H. Lee, Phys. Rev. B 50, 10788 (1994).

[54] M. R. Zirnbauer, Ann. Phys. (Leipzig) 3, 513 (1994).

[55] D.-H. Lee and Z. Wang, Philos. Mag. Lett. 73, 145 (1996).

[56] J. Kondev and J.B. Marston, Nucl. Phys. B 497, 639 (1997).

[57] M. R. Zirnbauer, J. Math. Phys. 38, 2007 (1997) and 2197 (1999).

[58] J. B. Marston and S.-W. Tsai, Phys. Rev. Lett. 82, 4906 (1999).

[59] I. Affleck, Nucl. Phys. B 265, 409 (1986).

[60] I. A. Gruzberg, A. W. W. Ludwig, and N. Read, Phys. Rev. Lett. 82, 4524 (1999).

[61] J. Cardy, Phys. Rev. Lett. 84, 3507 (2000). 
[62] E. J. Beamond, J. Cardy, and J. T. Chalker, Phys. Rev. B 65, 214301 (2002).

[63] A. D. Mirlin, F. Evers, and A. Mildenberger, J. Phys. A 36, 3255 (2003).

[64] J. Cardy, Commun. Math. Phys. 258, 87 (2005).

[65] F. J. Dyson, Phys. Rev. 92, 1331 (1953).

[66] E. R. Smith, J. Phys. C 3, 1419 (1970).

[67] G. Theodorou and M. H. Cohen, Phys. Rev. B 13, 4597 (1976).

[68] L. Fleishman and D. C. Licciardello, J. Phys. C 10, L125 (1977).

[69] A. A. Ovchinnikov and N. S. Érikhman, Zh. Eksp. Teor. Fiz. 73, 650 (1977) [Sov. Phys. JETP 46, 340 (1977)].

[70] V. L. Berezinskii, Zh. Eksp. Teor. Fiz. 65, 1251 (1973) [Sov. Phys. JETP 38, 620 (1974)].

[71] A. A. Gogolin and V. I. Melnikov, Zh. Eksp. Teor. Fiz. 73, 706 (1977) [Sov. Phys. JETP 46, 369 (1977)]; see also M. Steiner, M. Fabrizio, and A. O. Gogolin, Phys. Rev. B 57, 8290 (1998).

[72] T. P. Eggarter and R Riedinger, Phys. Rev. B 18, 569 (1978).

[73] A. D. Stone, and J. D. Joannopoulos, Phys. Rev. B 24, 3592 (1981).

[74] E. Witten, Nucl. Phys. B 188, 513 (1981).

[75] J. P. Bouchaud, A. Comtet, A. Georges, and P. Le Doussal, Ann. Phys. (N.Y.) 201, 285 (1990); A. Comtet, J. Desbois, and C. Monthus, Ann. Phys. (NY) 239, 312 (1995).

[76] D. G. Shelton and A. M. Tsvelik, Phys. Rev. B 57, 14242 (1998).

[77] L. Balents and M. P. A. Fisher, Phys. Rev. B 56, 12970 (1997).

[78] M. Bocquet, Nucl. Phys. B 546, 621 (1999).

[79] I. A. Gruzberg, N. Read, and A. W. W. Ludwig, Phys. Rev. B 63, 104422 (2001).

[80] O. Motrunich, K. Damle, and D. A. Huse, Phys. Rev. B 65, 064206 (2002).

[81] C. Mudry, S. Ryu, and A. Furusaki, Phys. Rev. B 67, 064202 (2003).

[82] P. W. Brouwer, C. Mudry, and A. Furusaki, Phys. Rev. Lett. 84, 2913 (2000).

[83] M. Titov, P. W. Brouwer, A. Furusaki, and C. Mudry, Phys. Rev. B 63, 235318 (2001).

[84] O. N. Dorokhov, Pis'ma Zh. Eksp. Teor. Fiz. 36, 259 (1982) [JETP Letters 36, 318]; P. A. Mello, P. Pereyra, and N. Kumar, Ann. Phys. (NY) 181, 290 (1988).

[85] C. Mudry, P. W. Brouwer, and A. Furusaki, Phys. Rev. B 59,13221 (1999).

[86] A. Hüffmann: J. Phys. A 23, 5733 (1990).

[87] P. W. Brouwer, C. Mudry, and A. Furusaki, Nucl. Phys. B 565, 653 (2000); P. W. Brouwer, A. Furusaki, C. Mudry, and S. Ryu, BUTSURI 60, 935 (2005) (see cond-mat/0511622 for english version).

[88] P. W. Brouwer, A. Furusaki, I. A. Gruzberg, and C. Mudry, Phys. Rev. Lett. 85, 1064 (2000).

[89] O. Motrunich, K. Damle, and D. A. Huse, Phys. Rev. B 63, 224204 (2001).

[90] I. A. Gruzberg, N. Read, and S. Vishveshwara, Phys. Rev. B 71, 245124 (2005).

[91] J. E. Bunder and R. H. McKenzie, Nucl. Phys. B 592, 445 (2001).

[92] M. Abramowitz and I. A. Stegun, Handbook of Mathematical Functions, Dover, New-York (1965).

[93] I. S. Gradshteyn and I. M. Ryzhik, Tables of Integrals, Series, and Products, 5th Ed., Academic Press, London (1994).

[94] L. Frappat, A. Sciarrino, and P. Sorba, Dictionary on Lie Algebras and superalgebras, (Academic Press, London 2000).

[95] B. DeWitt, Supermanifolds, Second edition, (Cambridge University Press, New York 1992).

[96] P. G. O. Freund, Supersymmetry, (Cambridge University Press, New York 1986).

[97] Y.-Z. Zhang and M. D. Gould, J. Math. Phys. 46, 013505 (2005).

[98] G. Götz, T. Quella, and V. Schomerus, J. Algebra 312, 829 (2007).

[99] L. Frappat, V. Hussin, and G. Rideau, J. Phys. A: Math Gen. 31, 4049 (1998). 